\title{
Characterization of Residual Chlorinated Organic Compounds in the Soil and Sediment at the Wilson's Corner Site
}

by

R. L. Nichols

Westinghouse Savannah River Company

Savannah River Site

Aiken, South Carolina 29808

T. R. Jarosch

K. M. Jerome

DOE Contract No. DE-AC09-96SR18500

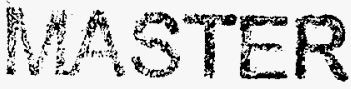

This paper was prepared in connection with work done under the above contract number with the U.S.

Department of Energy. By acceptance of this paper, the publisher and/or recipient acknowledges the U.S. Government's right to retain a nonexclusive, royalty-free license in and to any copyright covering this paper, along with the right to reproduce and to authorize others to reproduce all or part of the copyrighted paper. 


\section{DISCLAIMER}

This report was prepared as an account of work sponsored by an agency of the United States Government. Neither the United States Government nor any agency thereof, nor any of their employees, makes any warranty, express or implied, or assumes any legal liability or responsibility for the accuracy, completeness, or usefulness of any information, apparatus, product, or process disclosed, or represents that its use would not infringe privately owned rights. Reference herein to any specific commercial product, process, or service by trade name, trademark, manufacturer, or otherwise does not necessarily constitute or imply its endorsement, recommendation, or favoring by the United States Government or any agency thereof. The views and opinions of authors expressed herein do not necessarily state or reflect those of the United States Government or any agency thereof.

This report has been reproduced directly from the best available copy.

Available to DOE and DOE contractors from the Office of Scientific and Technical Information, P.O. Box 62, Oak Ridge, TN 37831; prices available from (615) 576-8401.

Available to the public from the National Technical Information Service, U.S. Department of Commerce, 5285 Port Royal Road, Springfield, VA 22161. 


\section{DISCLAIMER}

Portions of this document may be illegible electronic image products. Images are produced from the best available original document. 


\section{Characterization OF RESIDUAL \\ Chlorinated ORGanic CompoundS \\ IN THE SOIL AND SEDIMENT \\ AT THE WILSON'S CORNER SITE (U)}

January, 1997

R. L. Nichols

T. R. Jarosch

K. M. Jerome

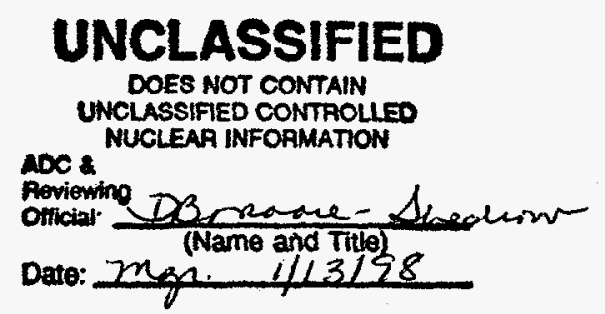

Westinghouse Savannah River Company

Savannah River Site

Aiken, SC

Prepared for the National Aeronautics and Space Administration under NASA HQ Order \# W-18,895 


\section{Summary}

Depth discrete bulk sediment samples were collected from 15 different locations at the Wilson's Corner site on the Kennedy Space Center during August 1996. Approximately 800 feet of continuous core and 800 sediment samples were collected. Four hundred of the samples were analyzed for chlorinated volatile organic compounds (CVOCs) using a modified version of EPA Method 5021 for headspace analysis of CVOCs in soil and water samples.

Sediments beneath the Wilson's Corner Site at the Kennedy Space Center contain residual trichloroethylene (TCE), cis-dichloroethylene (cDCE), vinyl chloride (VC), and Freon-113 (F113). Trichloroethylene has moved downward beneath source areas through the porous surficial aquifer along bedding planes until it encountered a silty sand and clay layer and then began to move laterally through the silty sand layer and along the top of the clay layer. Anaerobic biodegradation of trichloroethylene has produced cis-dichloroethylene and vinyl chloride along the migration path. The highest concentrations of CVOCs are immediately below the source areas and in a depression at the base of the surficial aquifer located south of the current stripper control room building.

Currently the Wilson's Corner Site has a network of recovery wells operating to maintain hydraulic control of the contamination and remove contaminant mass. Future remediation enhancements should focus on improved source term remediation and optimizing natural bioremediation, such as; Dual phase remediation of sediments in the source areas, installation of a deep recovery well near sampling location NASA1 where the depression in the surficial aquifer is located, air sparging at the leading edge of the plume to degrade biodegradation products, and targeted excavation of shallow contaminated soils. 


\section{Introduction}

The.Wilson's Corner site is located east of Titusville, Florida on the Kennedy Space Center (KSC) on the north side of State Route 402. Wilson's Corner was the location of the Propellant Systems Components Laboratory which was constructed in 1963. In addition to the laboratory building a facility for solvent cleaning of components such as rocket fuel lines was located east of the main building. Solvent storage tanks were located north of the main building.

In January 1986 moderate to low concentrations of CVOCs were reported for 3 monitoring wells located at the Wilson's Corner site. A groundwater remediation system for the contamination at the Wilson's Corner site was constructed and began operation in 1989 . The current system is stabilizing the groundwater contamination and removing dissolved contamination. Environmental personnel at $\mathrm{KSC}$ noticed that the CVOC concentrations in the recovered groundwater had leveled off at concentrations above their remediation goals and decided to investigate ways to enhance the removal of CVOCs. All previous characterization studies focused on groundwater sampling and as a result there was very little information on the residual contamination in the soil and sediments beneath the Wilson's Corner site. In 1994 KSC contacted the Savannah River Technology Center (SRTC) regarding the innovative technologies that had been studied at the Integrated Demonstration Site on the Savannah River Site. Following the discussions, KSC requested SRTC to conduct a detailed study of the residual contamination at the Wilson's Corner site and to assist in identifying potential enhancements to the existing remediation system. This report presents the results of a detailed study of the residual CVOC contamination in the soil and sediment at the Wilson's Corner site.

\section{Sampling and Analysis}

An innovative method for the sampling and analysis of CVOCs (Looney et al, 1993) in soil and groundwater, that was developed by the SRTC, was used to study the residual CVOC contamination in the soil and sediment at the Wilson's Corner site. This method has subsequently been refined by others, adopted and approved by the Environmental Protection Agency (EPA) as Method 5021 (Rev. 0, January 1995).

Depth discrete bulk sediment samples were collected from 15 different locations at the Wilson's Corner site on the Kennedy Space Center during August 1996, Figure. 1, Table 1. Continuous core was collected to a depth of 50 - 55 feet at each location using steam cleaned hollow stem augers and split spoon sampling tubes. The total depth at each core location was determined by the location of the confining layer at the base of the surficial aquifer. Once the core had been removed from the ground, $2-3 \mathrm{~cm}^{3}$ samples of the bulk sediment were immediately collected at 2 feet intervals and at significant lithologic changes using a modified plastic syringe and transferred to a $22.5 \mathrm{~mL}$ glass vials. $5 \mathrm{~mL}$ of deionized water was added to each vial as a suspending solution (giving a total sample volume of 7 to $8 \mathrm{~mL}$ ) and then a Teflon lined rubber septa and aluminum crimp top were placed on the bottle. All samples were refrigerated until analysis. After the depth discrete bulk sediment samples had been collected the geologist providing technical oversight of the drilling prepared a detailed lithologic description of the core, (Appendix A)

Prior to sampling the average weight of a $22.5 \mathrm{~mL}$ glass vial with $5 \mathrm{~mL}$ of pure deionized water and a Teflon lined rubber septa and aluminum crimp top (tare) was determined. The weight of the sediment sample was determined by weighing the sealed sample bottle and subtracting the average tare weight. 
Each sample was then analyzed using a-Hewlett Packard (HP) 5890 gas chromatograph (GC) equipped with an electron capture detector, a flame ionization detector, an HP 19395 headspace sampler, and a $60 \mathrm{~m}$ widebore glass capillary column (Supelco VOCOL ${ }^{\mathrm{TM}}$ ). The samples are heated to 70 degrees $\mathrm{C}$ in the autosampler prior to injection into the GC to maximize the transfer of CVOCs into the vapor phase (Looney et al, 1993). A complete set of standards in water $(7.5 \mathrm{~mL})$ was run with each set of samples for calibration. This method is a slightly modified version of the newly approved EPA Method 5021 for headspace analysis of CVOCs in soil and water samples.

The headspace method is best when used on sediment samples with minimal organic carbon content. High organic carbon content can produce results with lower than actual concentrations due to increased adsorption on the organic carbon. Several core locations had sediments with high organic carbon content in the 5 to 8 feet deep range and these results should be treated as suspect.

\section{Results}

Approximately 800 feet of continuous core and 800 sediment samples were collected. Four hundred of the samples were analyzed and a duplicate set was archived for future analysis as necessary. Results of the continuous coring indicate that the surficial aquifer is composed of a sandy shell hash and a silty medium to fine grained sand. The shell hash overlies the silty sand and varies in thickness from 8 - 25 feet. Occasionally the shell hash is present in 2 layers separated by fine grained sand. The surficial aquifer was deposited in a shore zone depositional environment and as a result has well developed bedding planes. The surficial aquifer is overlain by backfill, peat, and silty sand and is underlain by a silty clay layer approximately 50 feet deep, Figure 2 . The clay layer generally dips from east to west and has a local low in the center of the Wilson's Corner Site, Figure 3. On Figure 3 the color contours illustrate the structure of the top of the clay layer and the columns depict the cored locations and total sampling depth.

Trichloroethylene (TCE), cis-dichloroethylene (cDCE), vinyl chloride (VC), and Freon-113 (F113) were the primary CVOCs detected, Table 2. Breakdown products of F113 were also detected but not quantified due to a lack of calibration standards. Locations NASA3 and NASA5 had the highest concentrations of CVOCs as was expected since these locations are in the immediate vicinity of known source areas. CVOCs were present from the surface to the bottom of the surficial aquifer at NASA3 and NASA5. Figures $4-7$ show the trichloroethylene, cis-dichloroethylene, vinyl chloride and Freon-113 profiles for 4 sampling locations, NASA1, NASA3, NASA5 and NASA6. Results from the sample analysis vary over 5 orders of magnitude and as a result the concentration is plotted on a log scale in figures 4 7.

Sampling locations NASA3 and NASA5 are characteristic of source areas and.NASA1 and NASA6 are downgradient on the primary migration path. Trichloroethylene has migrated farther than Freon-113 has as shown by the presence of trichlorethylene in NASA 1 and NASA 6 and the lack of detectable Freon-113 in the same locations.

Data from the soil analysis was compiled into a contaminant model using 3 dimensional interpolation. The interpolation was performed using earthVision a product of Dynamic Graphics Inc. EarthVision uses a 3 dimensional minimum tension gridding algorithm to interpolate 3 dimensional data sets and can incorporate a vertical influence factor and 2 dimensional surfaces to constrain the model. In the Wilson's Corner model the vertical influence factor was used to increase the weighting in the lateral direction for interpolation to simulate the effect of bedding planes on contaminant migration. 
Two dimensional surfaces were prepared for the surface topography, water table, and top of the clay layer using data from the field work. The top and bottom of the contaminant model were constrained using the surface topography and top of the clay layer respectively.

The 3 dimensional migration yields a complex contamination pattern that is difficult to determine with conventional groundwater investigation methods. In Figure 8, a slice through the model reveals the pattern of contaminant migration beneath the equipment cleaning facility. The low concentrations at the bottom of the surficial aquifer beneath the source area in Figure 8 are due to contaminant removal from operation of recovery well NPSH-1. The high concentrations west of the source area in Figure 8 are in the depression in the clay layer at the base of the surficial aquifer. Note that cis-dichloroethylene and vinyl chloride have formed around the trichloroethylene in the depression and are migrating westward ahead of the trichloroethylene.

In Figure 9 you can see two primary contaminant source areas and the contamination that has resulted from downward and lateral migration. This pattern suggests that the contaminant moved downward through the porous surficial aquifer along bedding planes until it encountered the clay layer and then began to move laterally through a silty sand layer along the top of the clay layer. The lateral cross section through the model, at the base of Figure 9, reveals two areas of elevated TCE concentration just above the clay layer. The area of elevated TCE in the center of the site is the result of the migration of TCE from the sources along bedding planes and the top of the clay layer and into the depression that was shown in Figure 3. This is typical behavior of Dense Non-Aqueous Phase Liquids, (DNAPL) such as TCE. Again note that cis-dichloroethylene and vinyl chloride have formed around the trichloroethylene and are migrating westward ahead of the trichloroethylene. In addition, two aspects of the site remedial operations may enhance the pooling of contaminants in the region of the depression. First, there are no recovery wells at any depth in the vicinity of the depression to remove soluble contaminants. Second, the region is overlain by two major irrigation circuits which produce a vertical recharge gradient that may act to force or flush contaminants downward.

The trichloroethylene in the subsurface at Wilson's Corner has weathered forming cisdichloroethylene and vinyl chloride. The weathering is the result of in-situ anaerobic biodegradation. The weathering byproducts are most concentrated in the peat layer beneath the source areas, and beneath the trichloroethylene in the depression in the clay layer. The high organic carbon content of the peat has adsorbed large amounts of trichloroethylene and produces a strong reducing environment optimal for anaerobic biodegradation producing cisdichloroethylene and vinyl chloride. Vinyl chloride is resistant to further anaerobic biodegradation and will continue to accumulate in the subsurface unless it migrates into an aerobic environment where it can be degraded to $\mathrm{CO} 2, \mathrm{Cl}$, and water.

Trichloroethylene trapped in the depression has begun to biodegrade into cis: dichloroethylene and vinyl chloride. Cis-dichloroethylene and vinyl chloride that formed at the fringes of the trichloroethylene in the depression are migrating southwestward with the ambient groundwater flow toward recovery wells NPSH5 and NPSH20. No trichloroethylene was detected in NASA6 however, both cis-dichloroethylene and vinyl chloride were detected in NASA6 indicating that the leading edge of the trichloroethylene plume is currently biodegrading faster than it is moving. This data is consistent with monitoring results for well NPSH20 which has had no detectable trichloroethylene but has had cis-dichloroethylene and vinyl chloride. 


\section{Conclusions}

Sediments beneath the Wilson's Corner Site at the Kennedy Space Center contain residual trichloroethylene (TCE), cis-dichloroethylene (cDCE), vinyl chloride (VC), and Freon-113 (F113). The residual CVOCs are present in the following forms:

- Adsorbed to shallow peat layer and aquifer matrix

- Diffused into porous shell fragments and fine grained sediments

- Micro-droplets trapped in individual pore throats

Trichloroethylene moved downward beneath source areas through the porous surficial aquifer and along bedding planes until it encountered the clay layer and then began to move laterally along the top of the clay layer. Anaerobic biodegradation of trichloroethylene has produced cis-dichloroethylene and vinyl chloride along the migration path. The highest concentrations of CVOCs are immediately below the source areas and in the depression in the clay layer at the base of the surficial aquifer.

Currently the Wilson's Corner Site has a network of recovery wells operating to maintain hydraulic control of the contamination and remove contaminant mass. Future remediation enhancements should focus on improved source term remediation and optimizing natural bioremediation. Potential enhancements include:

- Dual phase remediation of sediments in the source areas. This would include installation of a shallow recovery well screened from 12 - 27 feet near NPSH1 and NPSH14 and pumping hard enough to lower the water table below the top of the screen. Vacuum extraction would be simultaneously performed on the shallow recovery well. Vacuum extraction will draw air downward through the contaminated peat layer and sediments and into the exposed well screen efficiently removing the volatile contaminants while the pumping will recover highly contaminated water from the most permeable zone at the Wilson's Corner Site.

- Install a deep recovery well near sampling location NASA1. After the dual phase remediation has been operated, a deep recovery well could be installed near NASA1 to remove the trichloroethylene that has migrated down to the base of the surficial aquifer preventing further diffusion into fine grained sediments and naturally migrating groundwater.

- Air sparging between NPSH 5 and NPSH20. Addition of air to the downgradient extent of the contamination would produce aerobic conditions capable of supporting biodegradation of cis-dichloroethylene and vinyl chloride.

- Targeted excavation and on-site management through biopiles. Limited excavation of shallow ( $<10$ feet deep) contaminated sediments at source areas that remain after dual phase remediation could be beneficial if the waste is managed on-site through biopiling. Biopiling is a cost effective method for on-site remediation of excavated soils containing volatile and biodegradable contaminants.

\section{References}

Looney, B. B., C. A. Eddy, and W. R. Sims, 1993. Evaluation of Headspace Method for Volatile Constituents in Soils and Sediments. I n Measuring and Interpreting VOCs in Soils: State of the Art and Research Needs, US Environmental Protection Agency, Environmental Monitoring Systems Laboratory, Las Vegas NV 89193. 
Table 1 Coordinates for sample locations at the Wilson's Corner Site.

\begin{tabular}{|c|c|c|c|}
\hline Location Id & Easting (feet) & Northing (feet) & Surf. Elev. (ft, msl) \\
\hline NASA1 & $595,657.98$ & $1,566,770.46$ & 7.25 \\
\hline NASA2 & $595,881.07$ & $1,566,703.44$ & 8.7 \\
\hline NASA3 & $596,070.49$ & $1,566,785.33$ & 8.14 \\
\hline NASA4 & $595,997.15$ & $1,566,881.07$ & 8.16 \\
\hline NASA5 & $595,813.02$ & $1,566,933.90$ & 7.7 \\
\hline NASA6 & $595,436.91$ & $1,566,715.65$ & 6.32 \\
\hline NASA7 & $595,521.68$ & $1,566,884.84$ & 7.24 \\
\hline NASA9 & $595,747.67$ & $1,566,552.52$ & 6.97 \\
\hline NASA10 & $595,176.13$ & $1,566,682.56$ & 5.53 \\
\hline NASA11 & $596,173.38$ & $1,567,003.41$ & 9.64 \\
\hline NASA14 & $595,833.11$ & $1,566,783.49$ & 8.52 \\
\hline NASA16 & $595,986.54$ & $1,566,975.14$ & 8.44 \\
\hline NASA17 & $595,333.69$ & $1,566,556.48$ & 4.99 \\
\hline NASA18 & $596,195.57$ & $1,566,867.68$ & 8.46 \\
\hline NASA19 & $595,225.22$ & $1,567,128.44$ & 7.15 \\
\hline
\end{tabular}

Table2 Summary of primary contaminants detected in bulk sediment samples at the Wilson's Corner Site.

\begin{tabular}{|l|c|c|l|}
\hline $\begin{array}{l}\text { Chlorinated } \\
\text { Volatile Organic } \\
\text { Compound }\end{array}$ & $\begin{array}{c}\text { Detection Limit } \\
\text { (ug/gm) }\end{array}$ & $\begin{array}{c}\text { Maximum } \\
\text { (ug/gm) }\end{array}$ & $\begin{array}{c}\text { Location of } \\
\text { Maximum }\end{array}$ \\
\hline Trichloroethylene & 0.001 & 19.37 & NASA3, 9 feet deep \\
\hline cis-Dichloroethylene & 0.001 & 87.36 & NASA5, 7 feet deep \\
\hline Vinyl Chloride & 0.001 & 6.45 & NASA5, 7 feet deep \\
\hline Freon 113 & 0.001 & 42.11 & NASA5, 7 feet deep \\
\hline
\end{tabular}




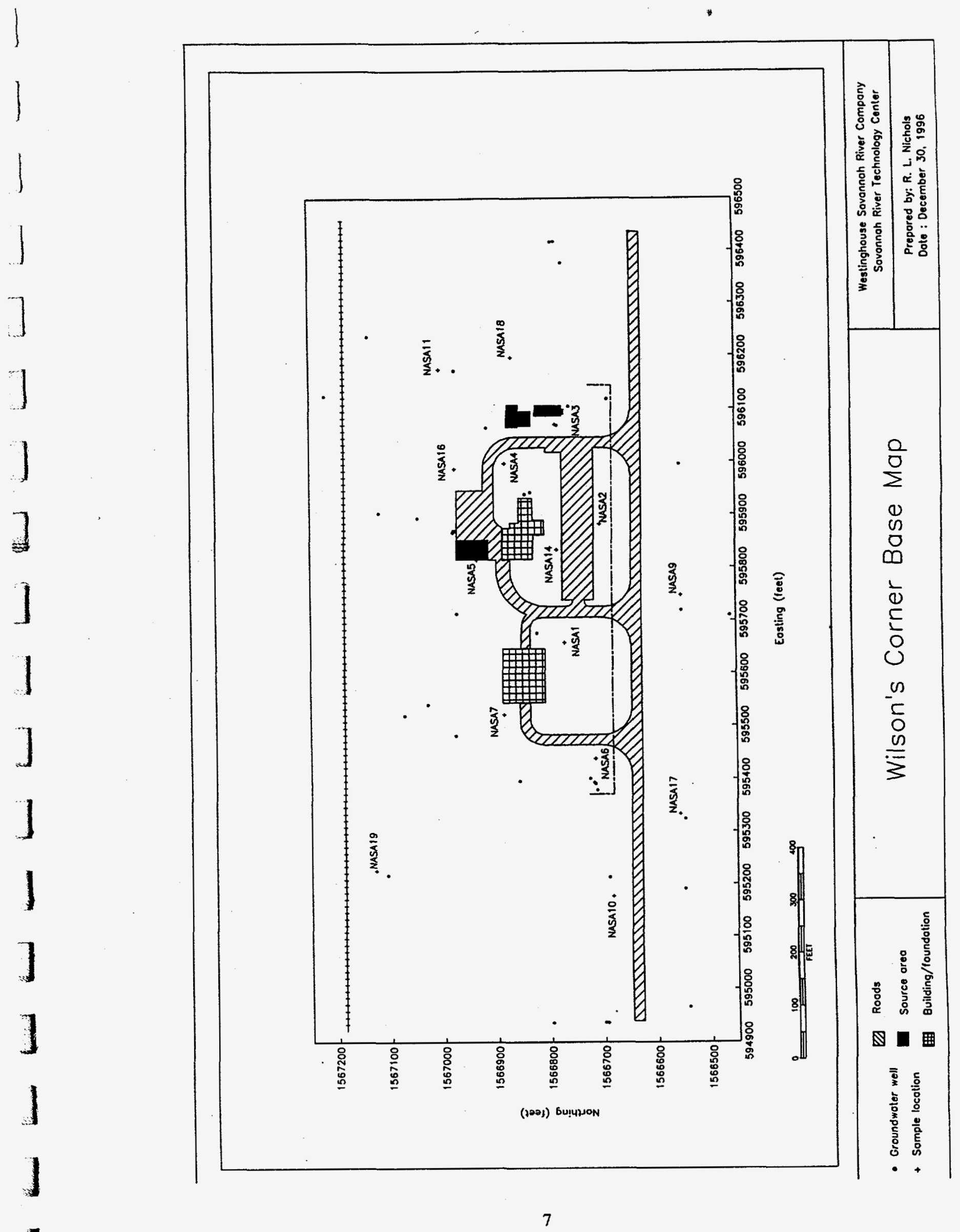


]

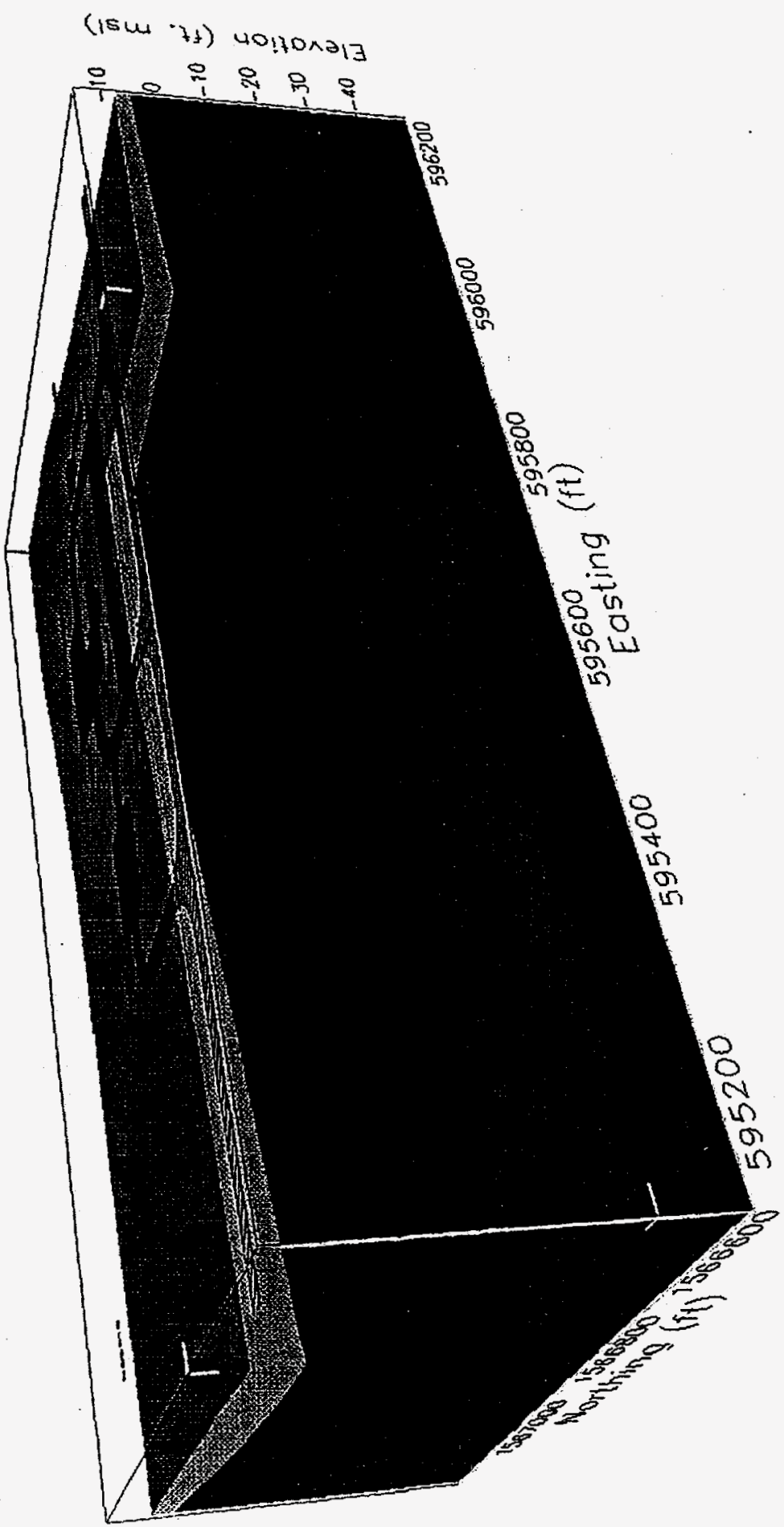

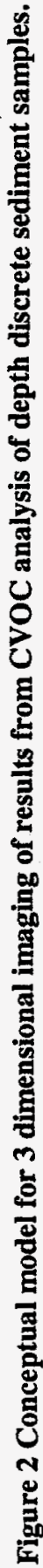

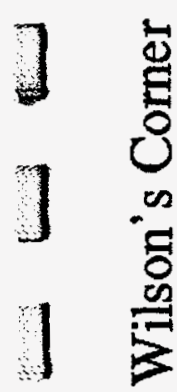

]

]

J

]

1

3

3

1 


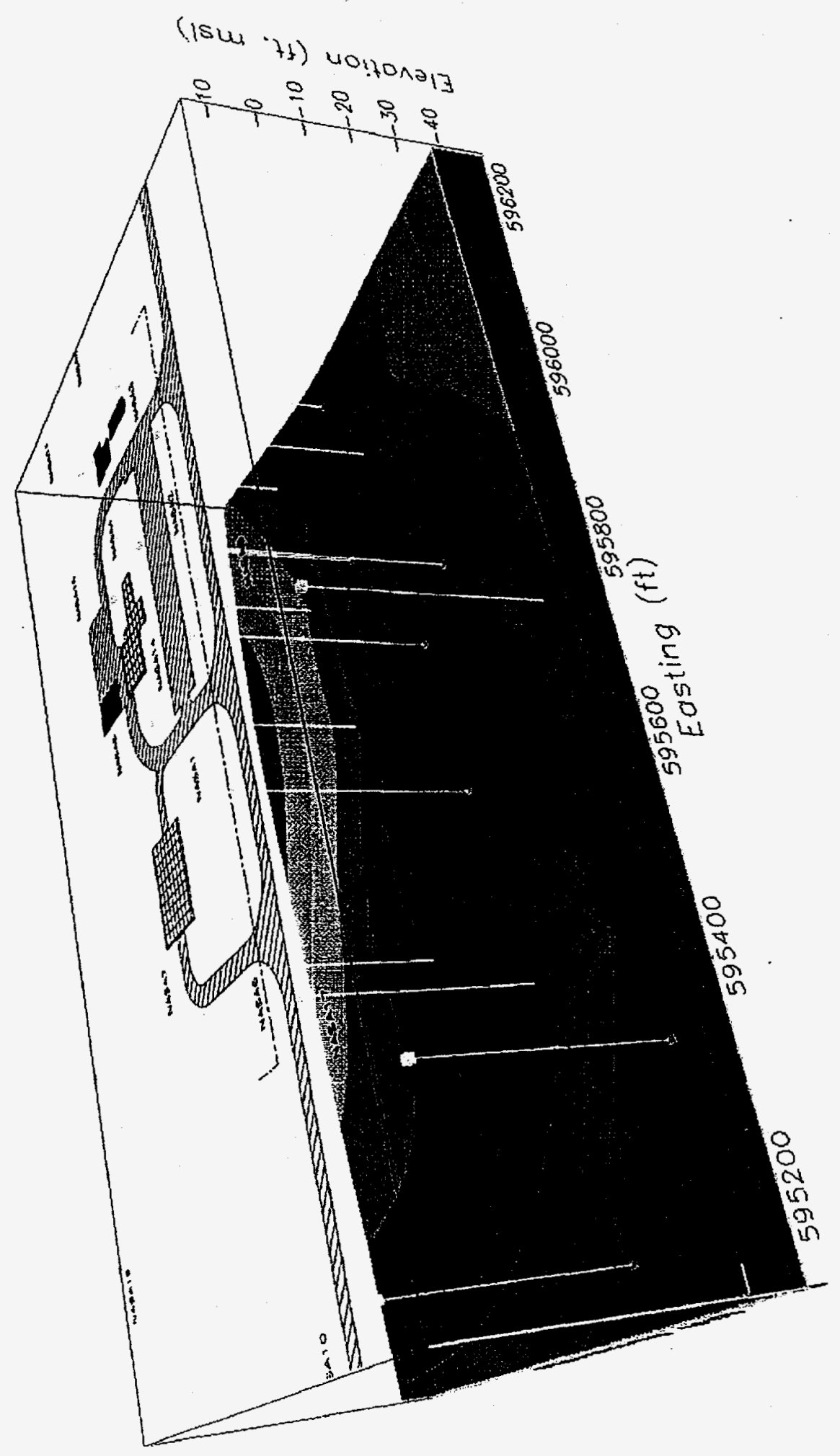

 

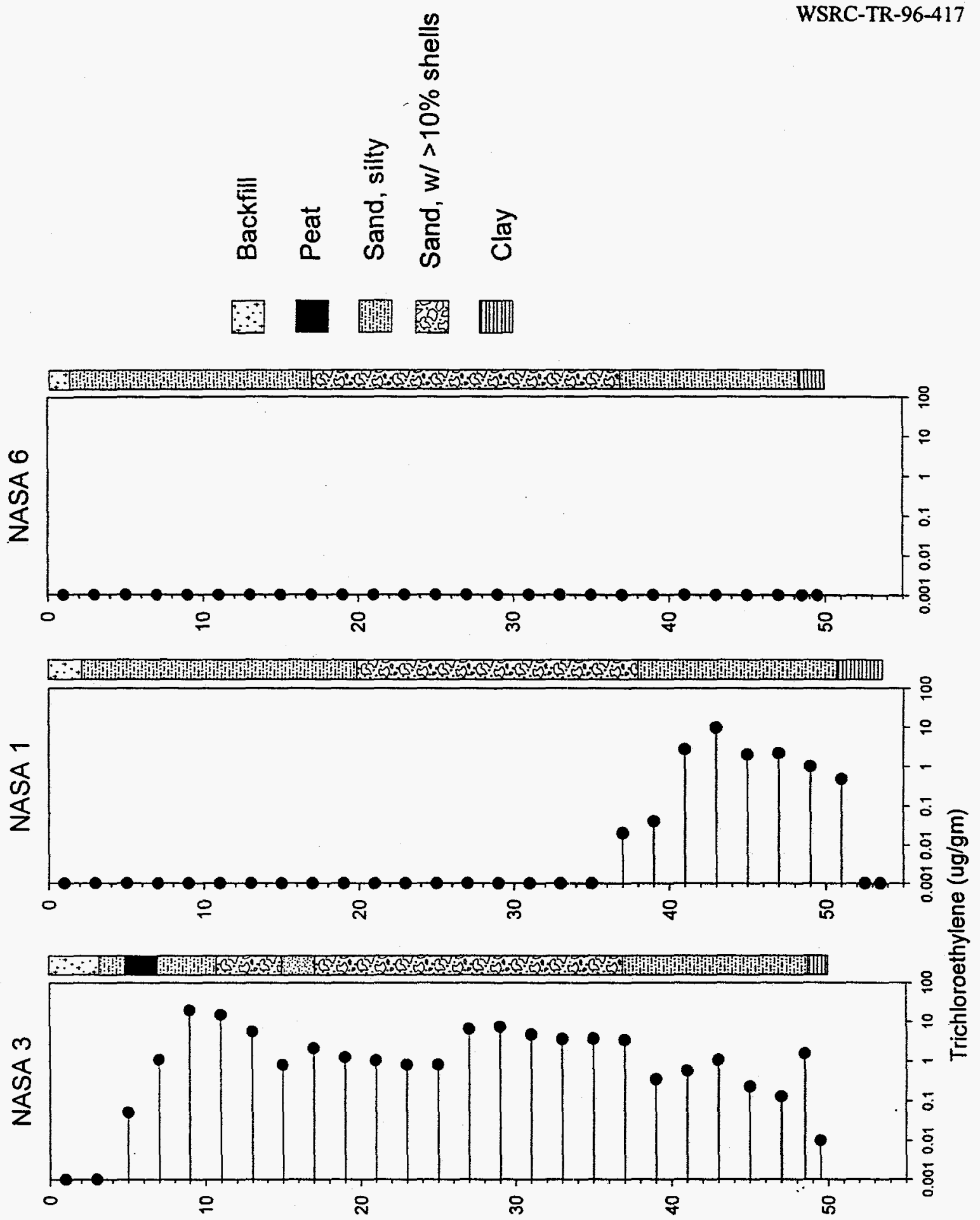

1

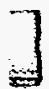

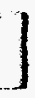

1

1

]

1

으 운

요

요

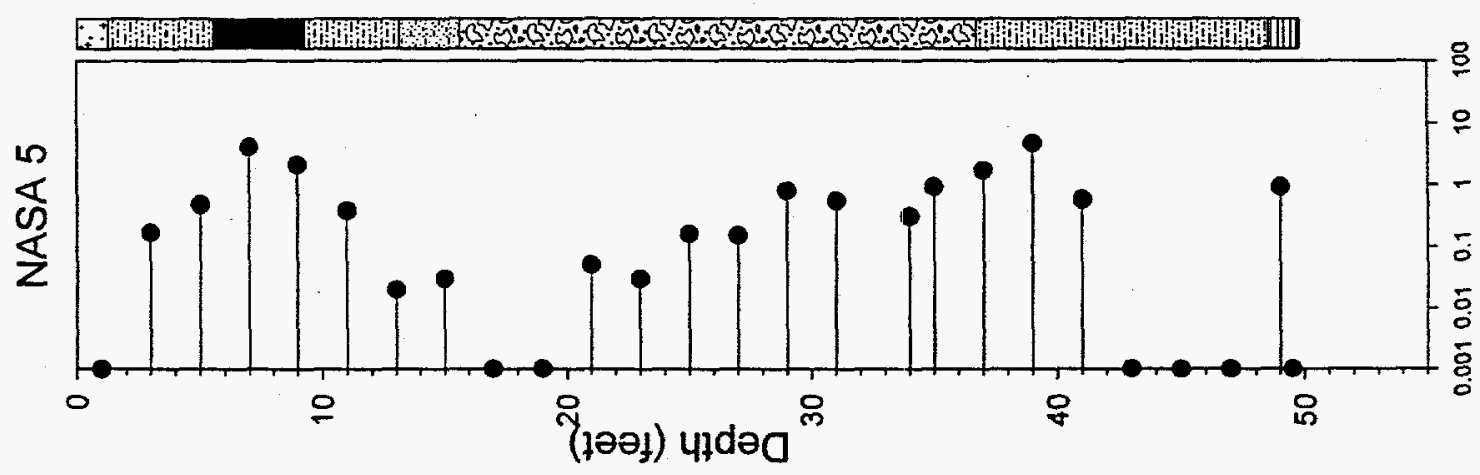


I
I
J
]
]
]
J
J
]
J
J
J

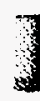

1

I

1
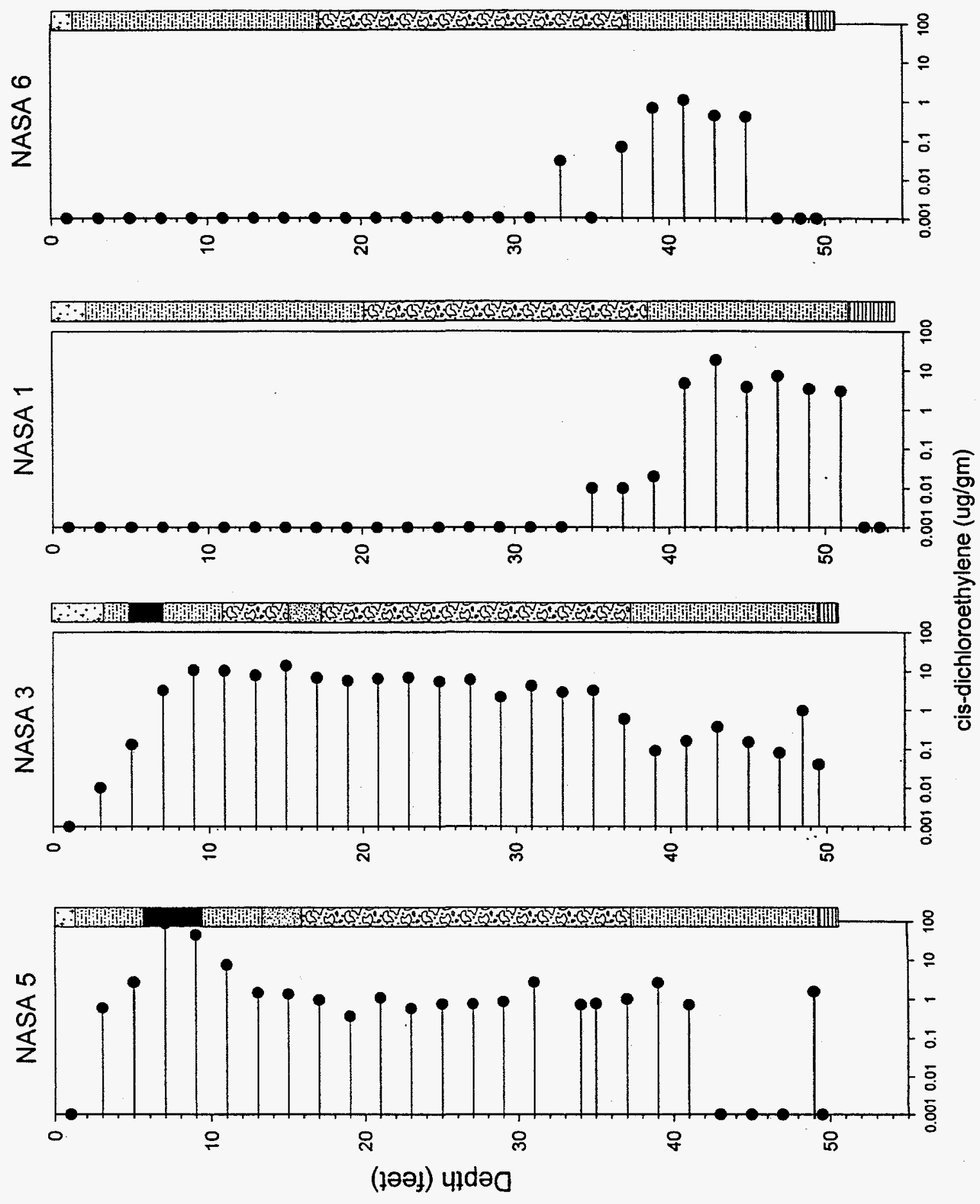


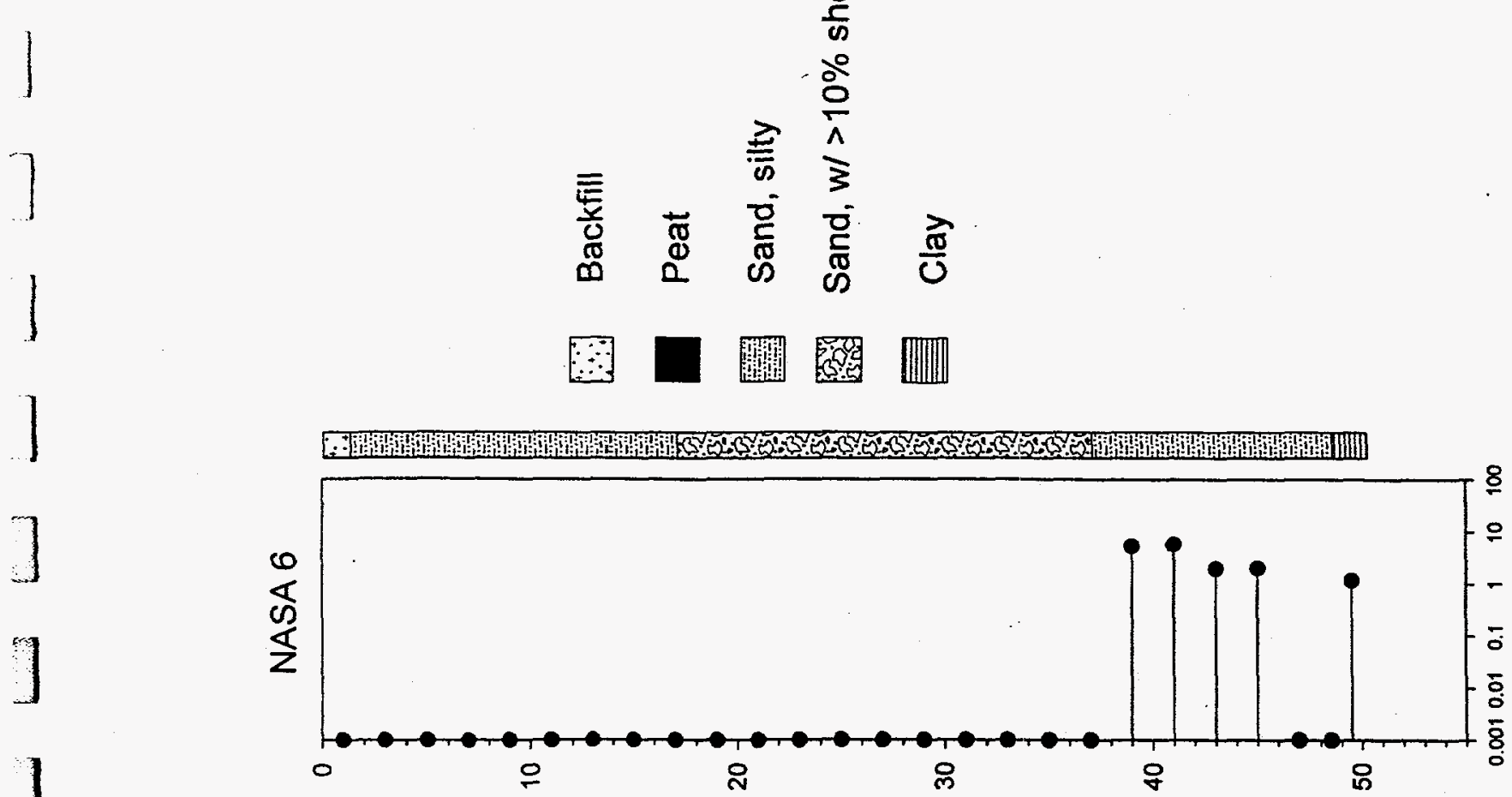

3

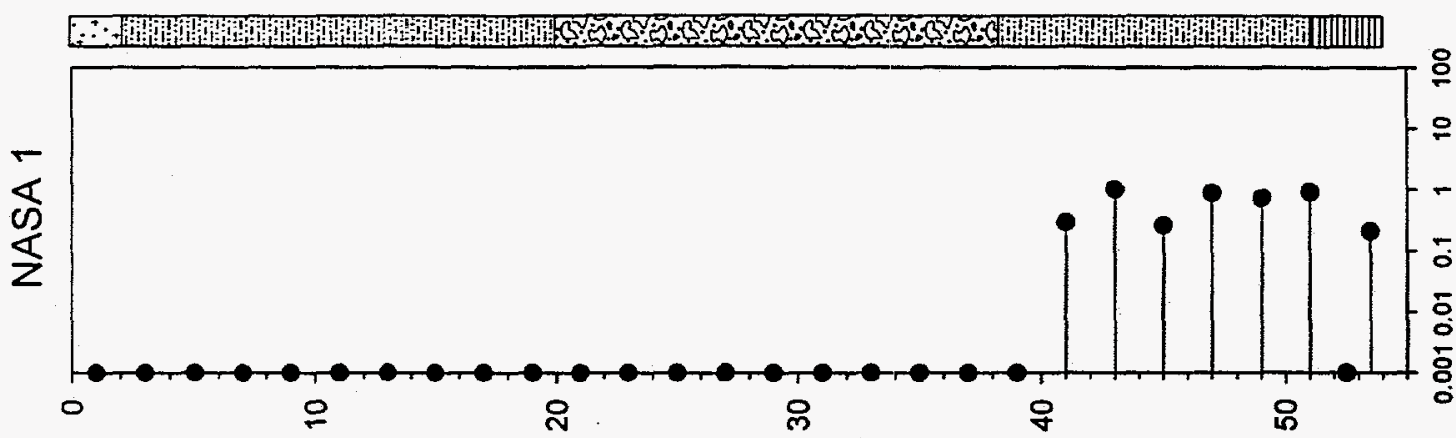

1

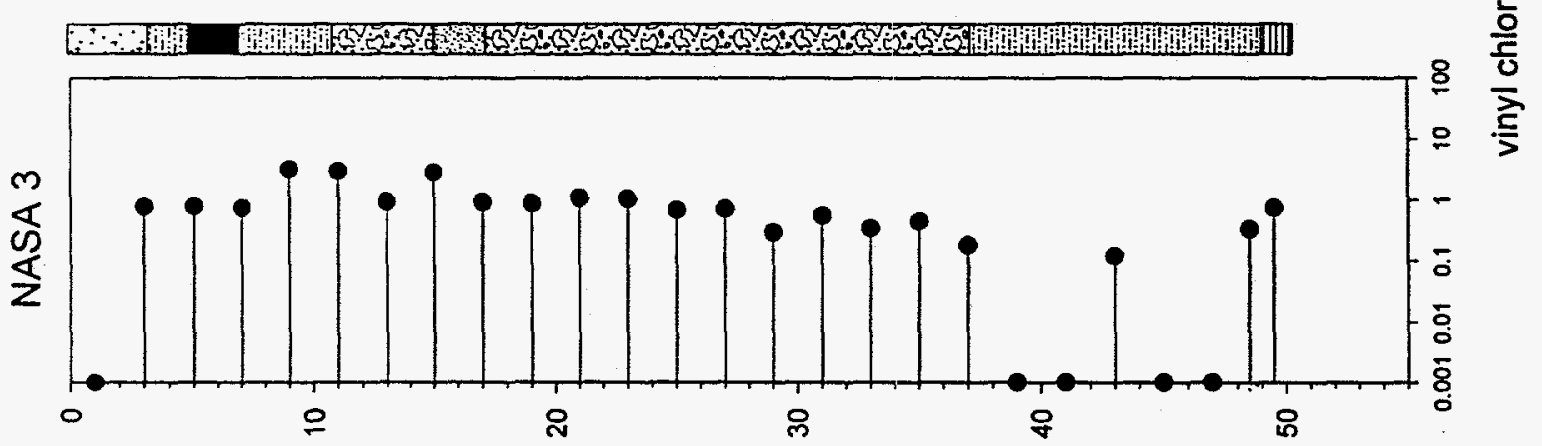

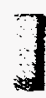

]

1

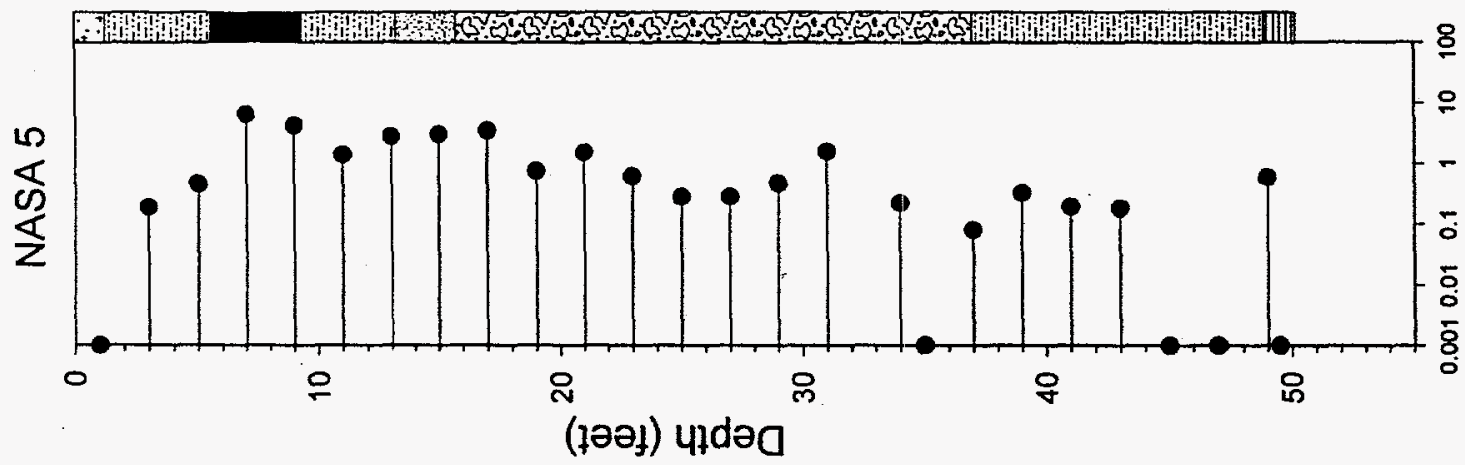




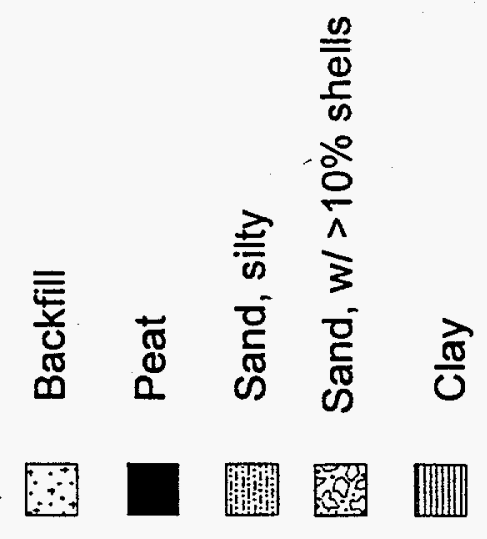

$\sqrt{7}$

Q

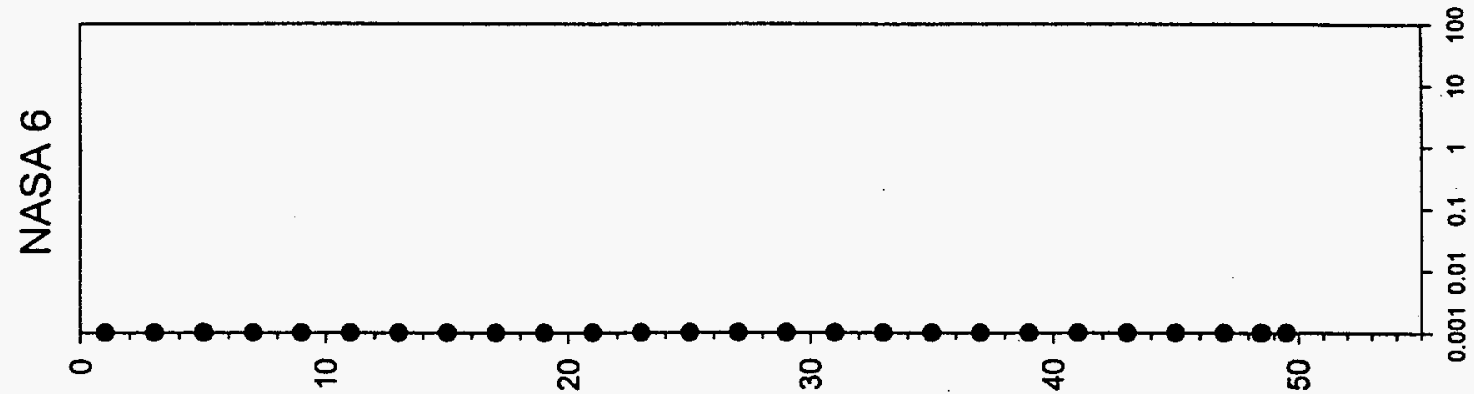

7
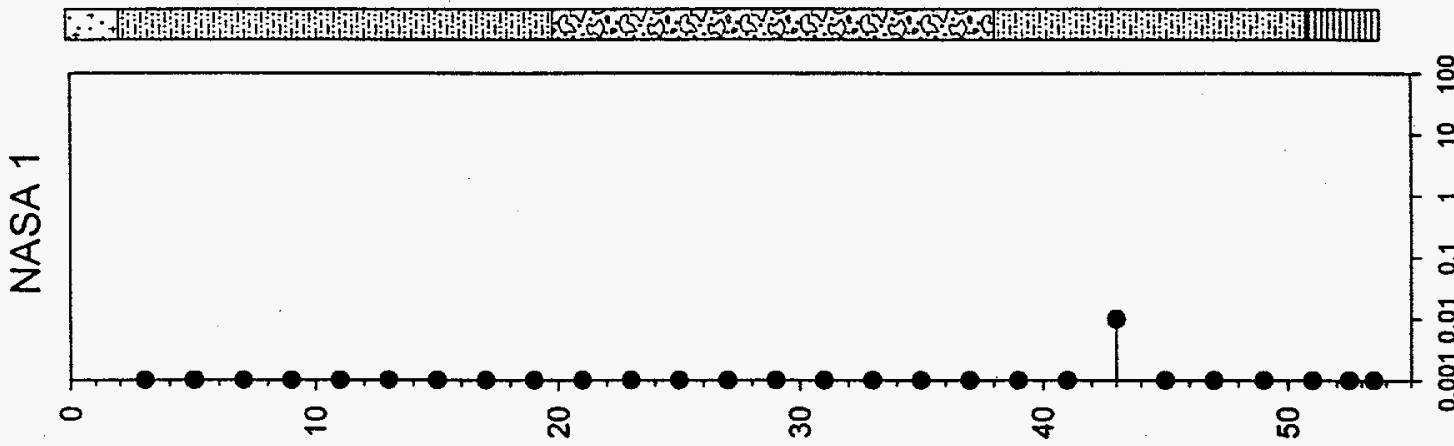

1

$\therefore \cdot \because$ Hiti

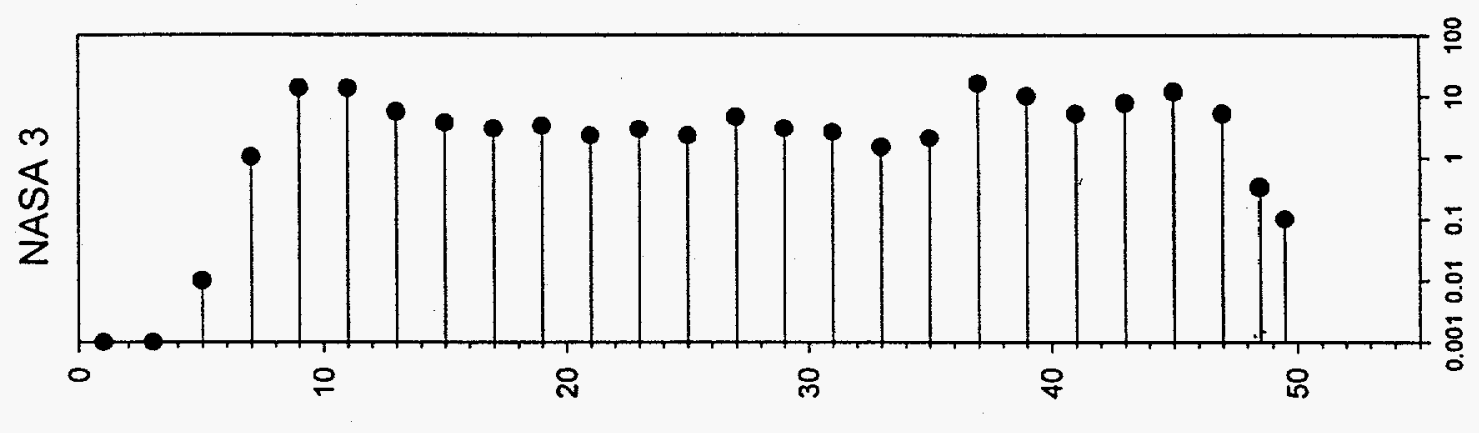

3

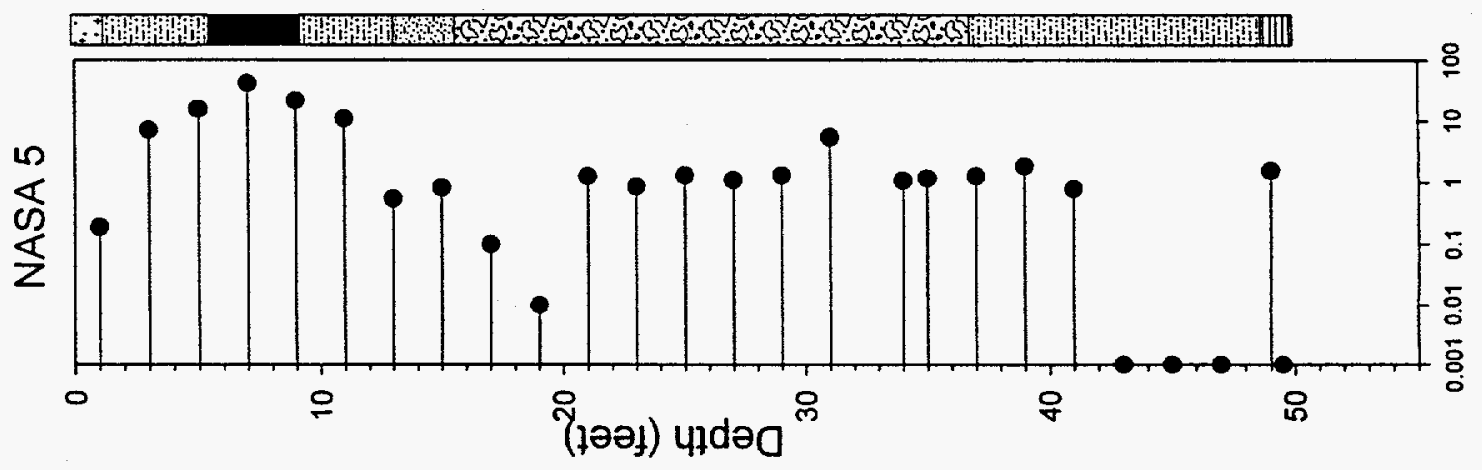

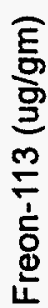

1

1

3

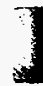

우 


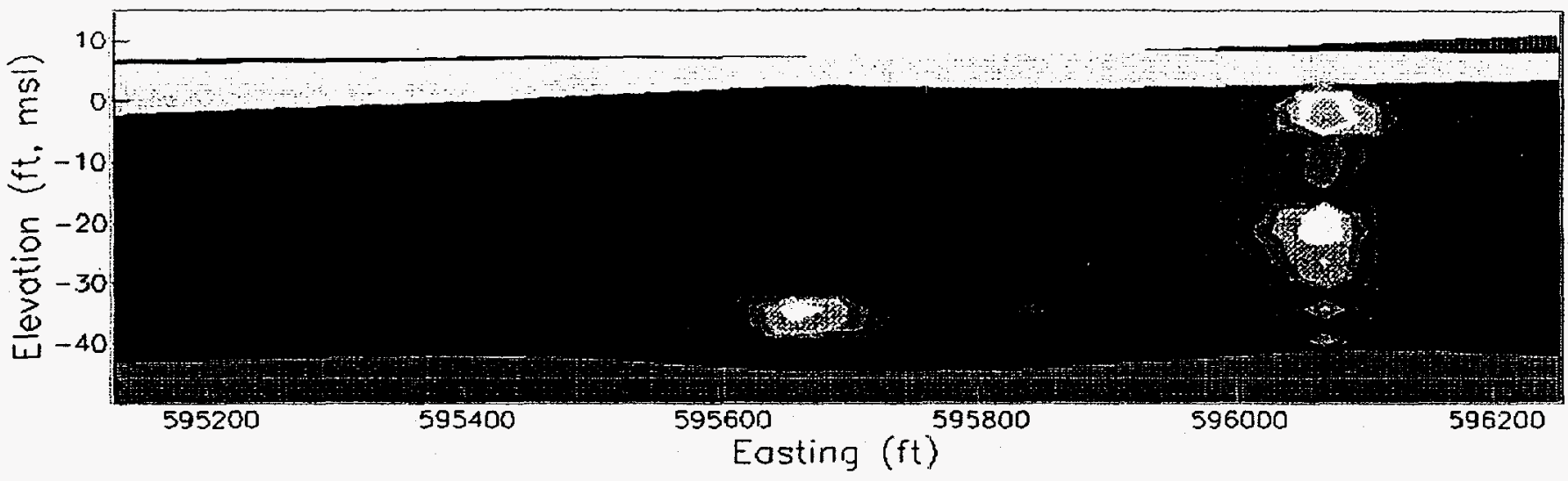

\section{cis-dichloroethylene}

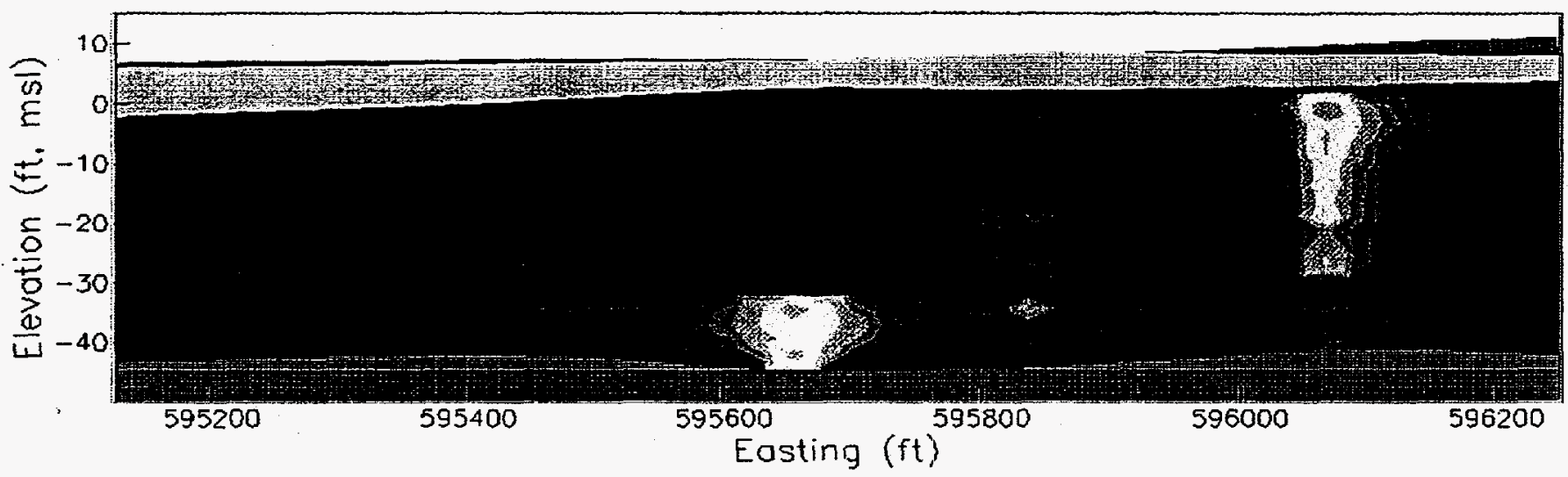

\section{vinyl chloride}
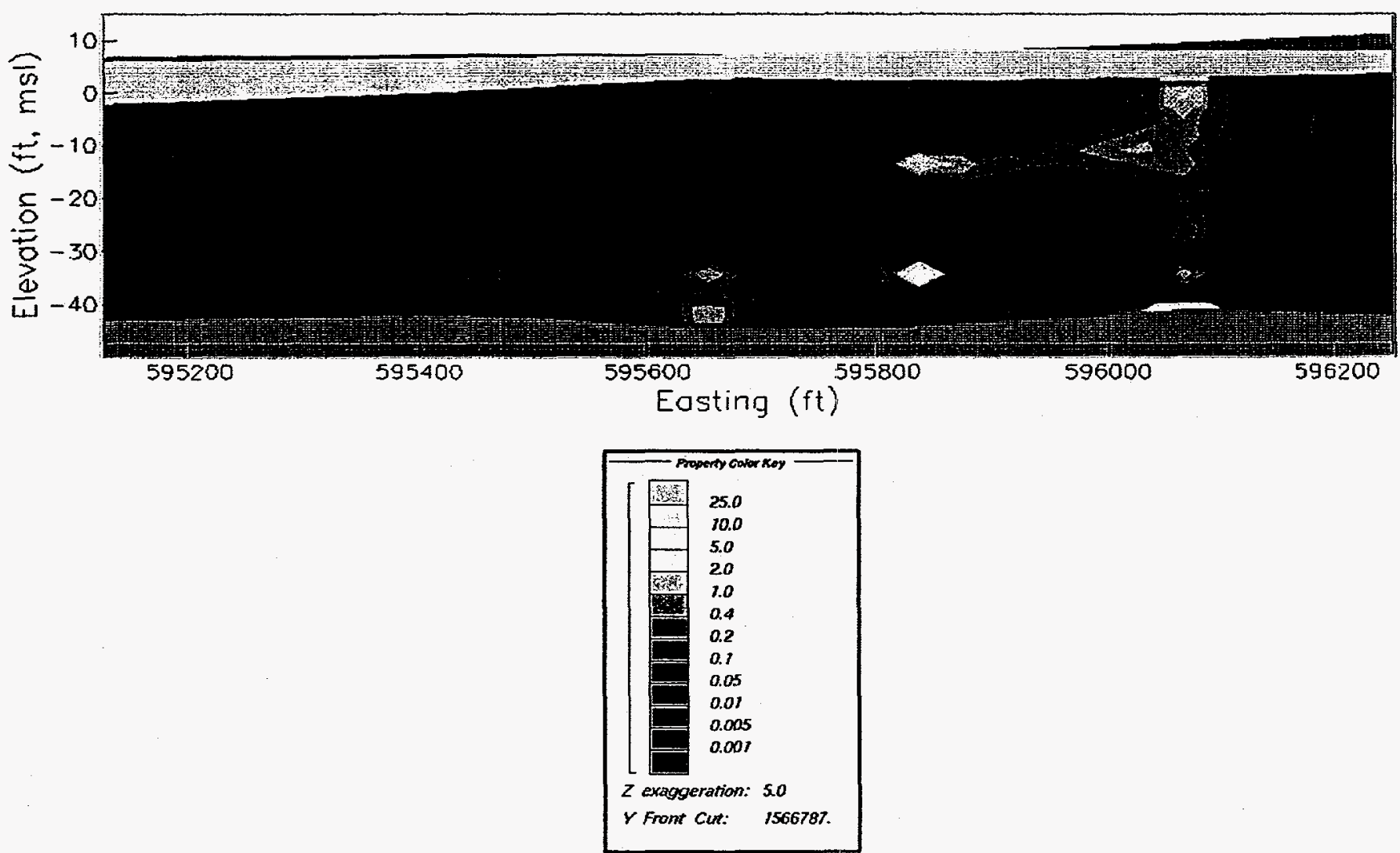

Figure 8 Cross section through 3 dimensional models of trichloroethylene, cis-dichloroethylene, and vinyl chloride results from headspace analysis of sediment samples. 


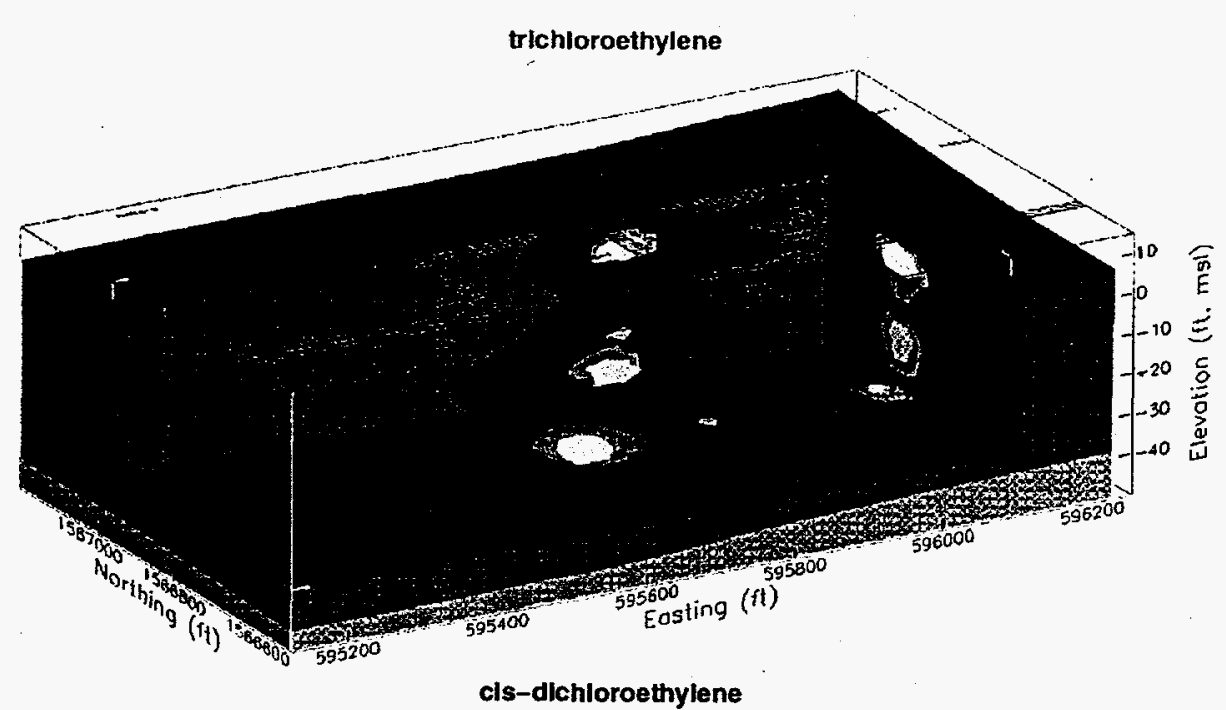

1
7

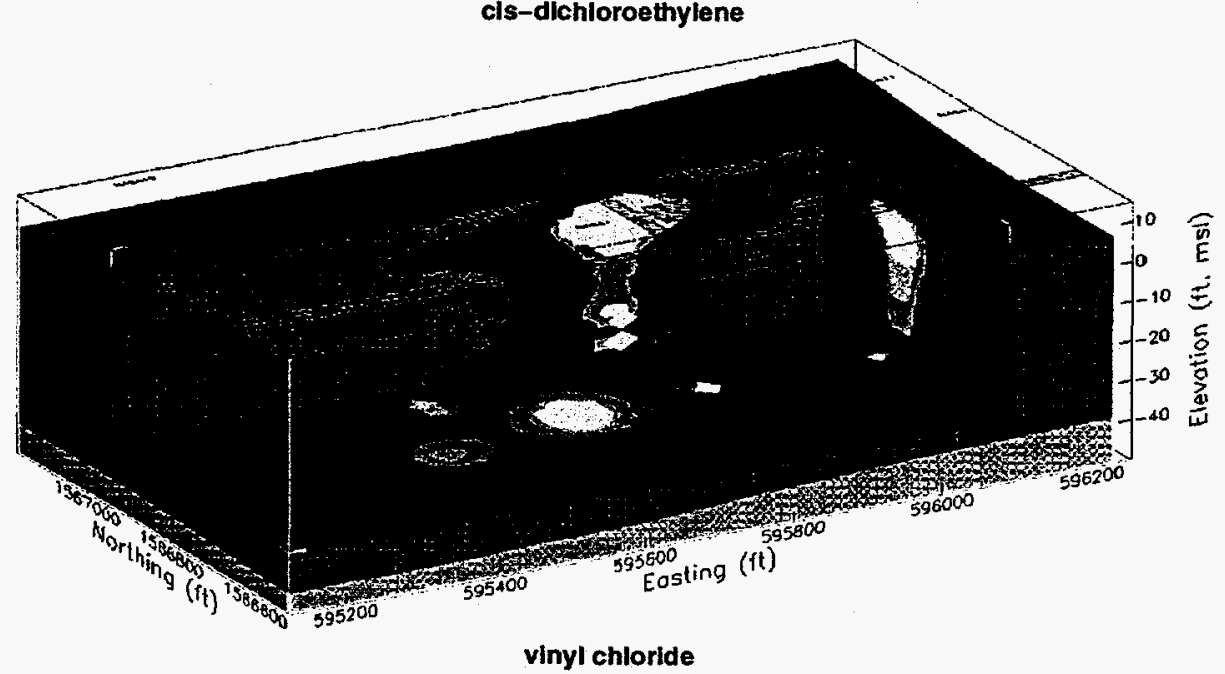

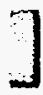

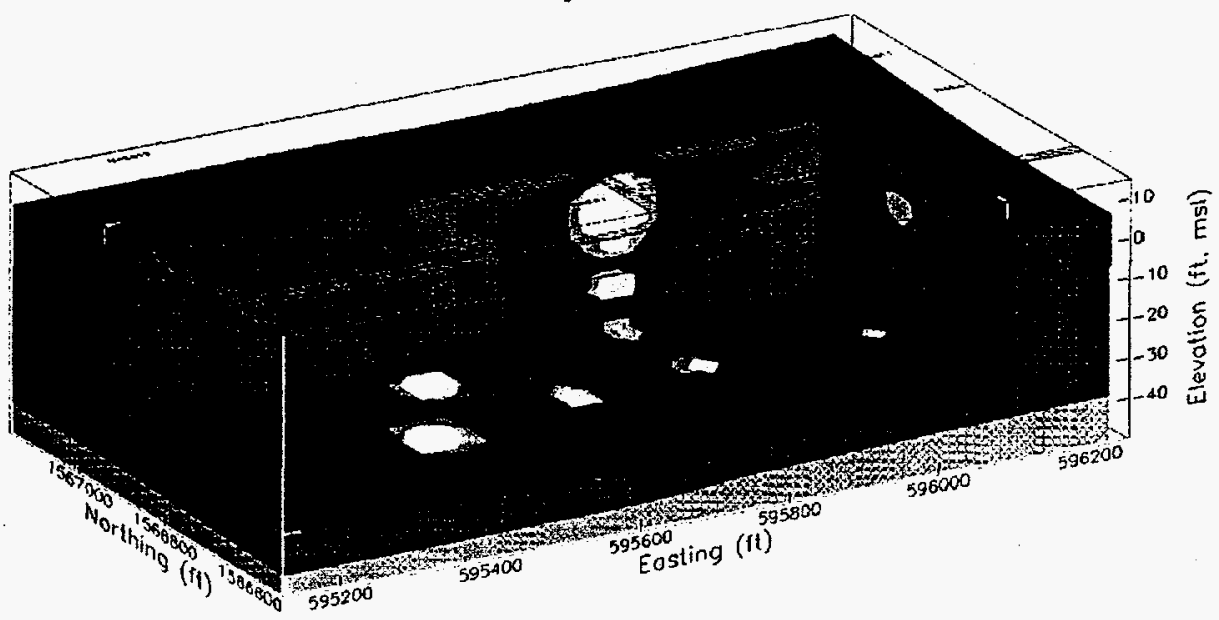

Figure 9 Three dimensional models of trichloroethylene, cis-dichloroethylene, and vinyl chloride results from headspace analysis of sediment samples showing the contaminant sources and migration along the top of the confining unit 
Appendix A

Soil Boring Logs 


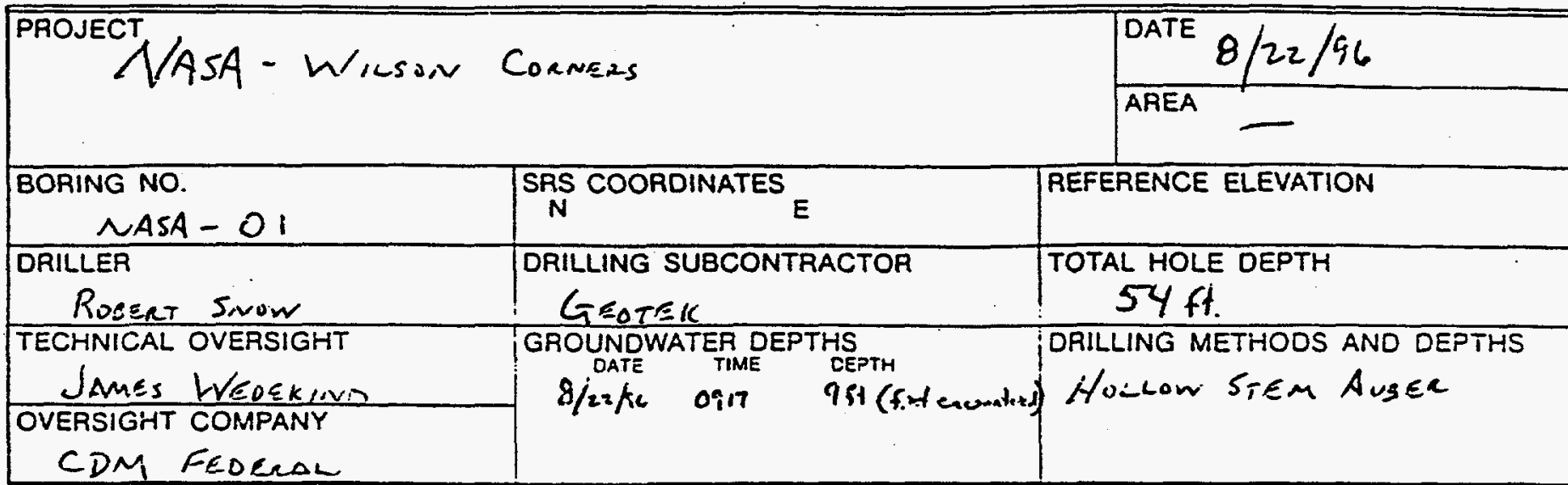

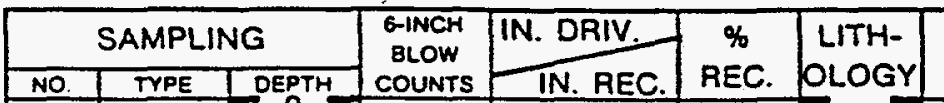

DESCRIPTION

GEASS AND TOPSOR with CRUSNEO stone
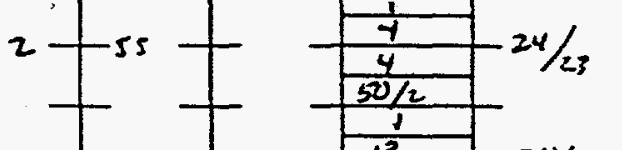

gecencs

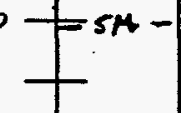

$3 \rightarrow$ ss 5

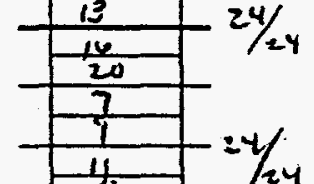

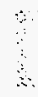

.

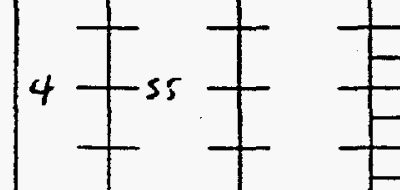

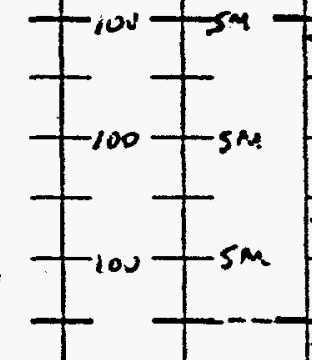

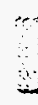

$\alpha$

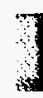

$$
5
$$

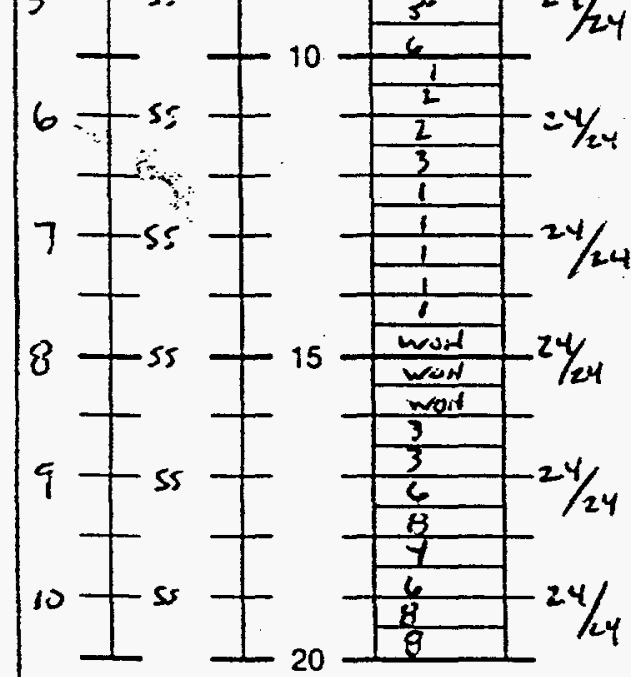

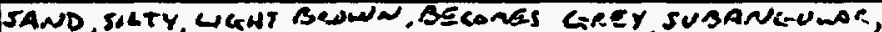

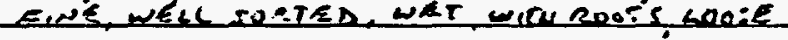

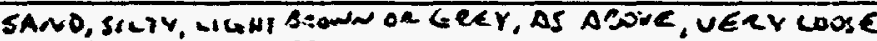
WET-Shevisid?

SANS SALY, BCOWN. BECONES CREY ar Devive

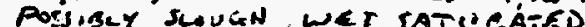

DER.NeTE rLOUGH

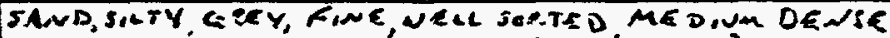
wird sufics, wNele, canse, Benkein

- Jcocati (sve oi sare)

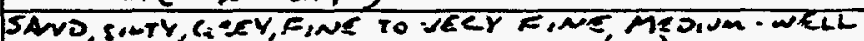

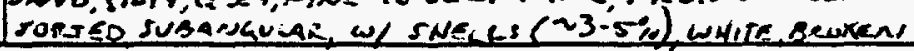




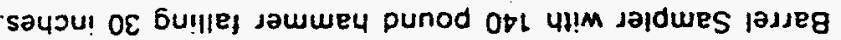
IIds *0.0. ट "0. jof smolg to wns s! ajueis!say vo!lenauad pjepueis : $: 10 \mathrm{~N}$

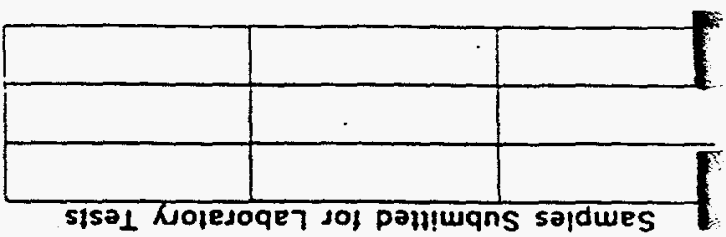

sisal Kolesoqe 101 pan!wqns sojdwes

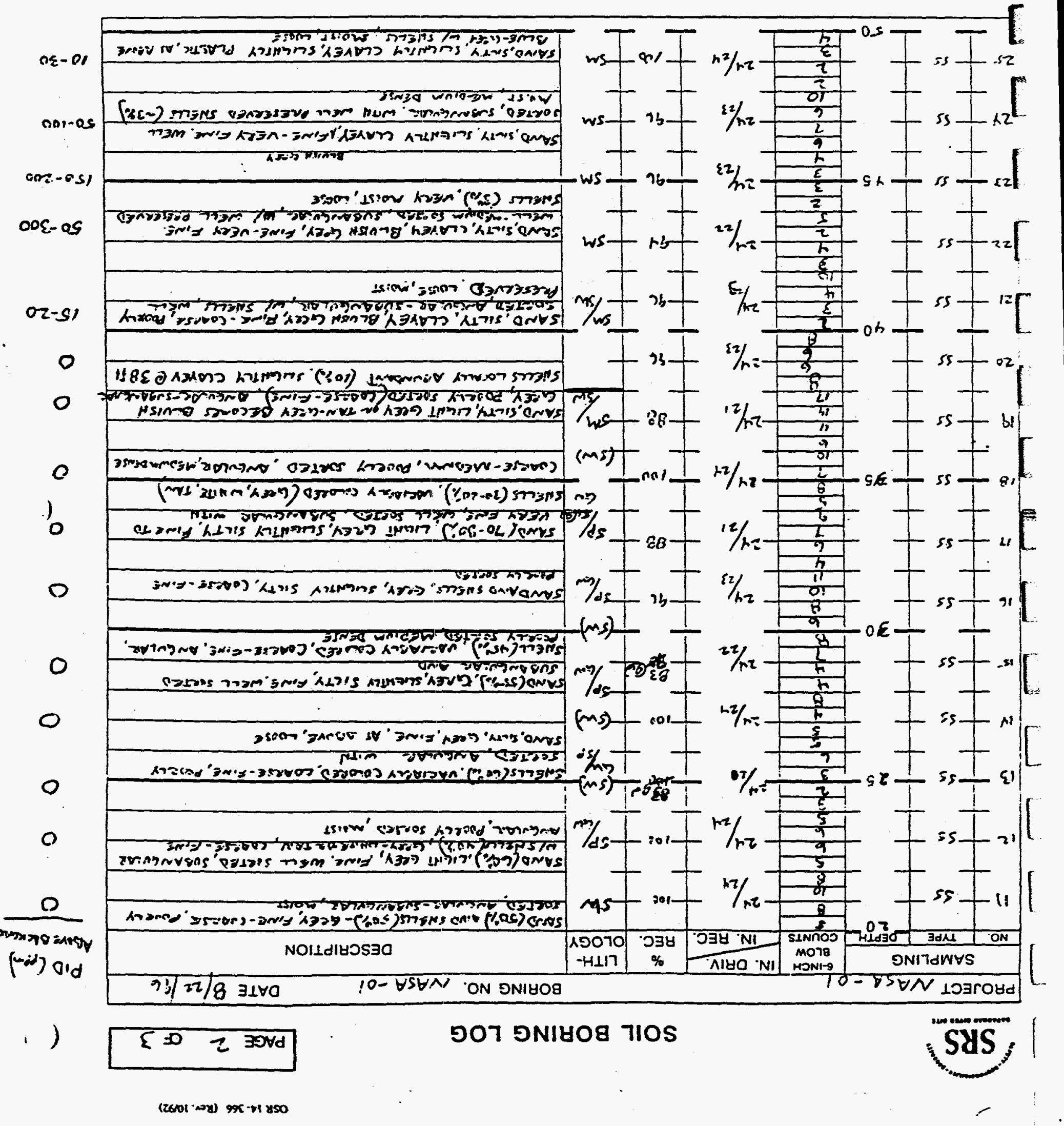




\section{SRS}

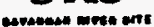

SOIL BORING LOG

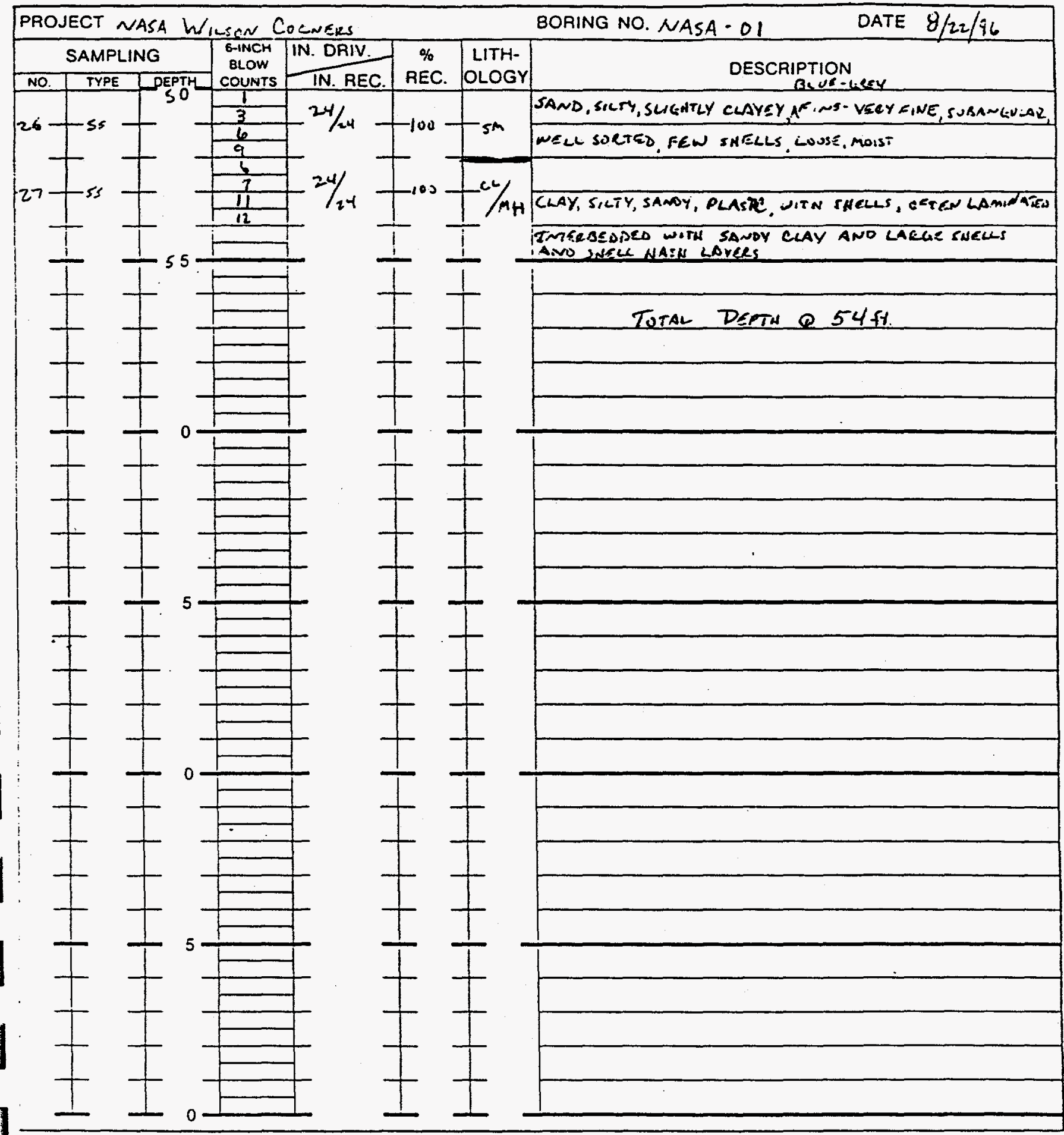

Samples Submitted for Laboratory Tesis
NOTE: Standard Penetration Resistance is Sum of Blows for 2nd - 6" and 3rd - 6" to Drive 1-3/8" 1.D., 2" O.D. Split Barrel Sampler with 140 pound hammer falling 30 inches 
PROJECT

$$
\begin{aligned}
& \text { NASA } \\
& \text { WILSONS COMNER fFL }
\end{aligned}
$$

$\begin{aligned} & \text { DATE } \\ & \text { AREA }\end{aligned} / 13 / 76$

BORING NO.

DRILLER

$$
\text { NASA O2 }
$$

Robert Snow

TECHNICAL OVERSIGHT

lames Wedekind

OVERSIGHT COMPANY

$C D M$ Federal

PRTS COORDINATES

DRILLING SUBCONTRACTOR

Gestek

GROUNDWATER DEPTHS

DATE TIME TERTH

$8 / 13 / 96 \quad 1425 \quad 28 \mathrm{ft}$
REFERENCE ELEVATION

TOTAL HOLE DEPTH

$54 \mathrm{ft}:$

DRILLING METHODS AND DEPTHS HSA\% CONzivusus SPLT SPOONS

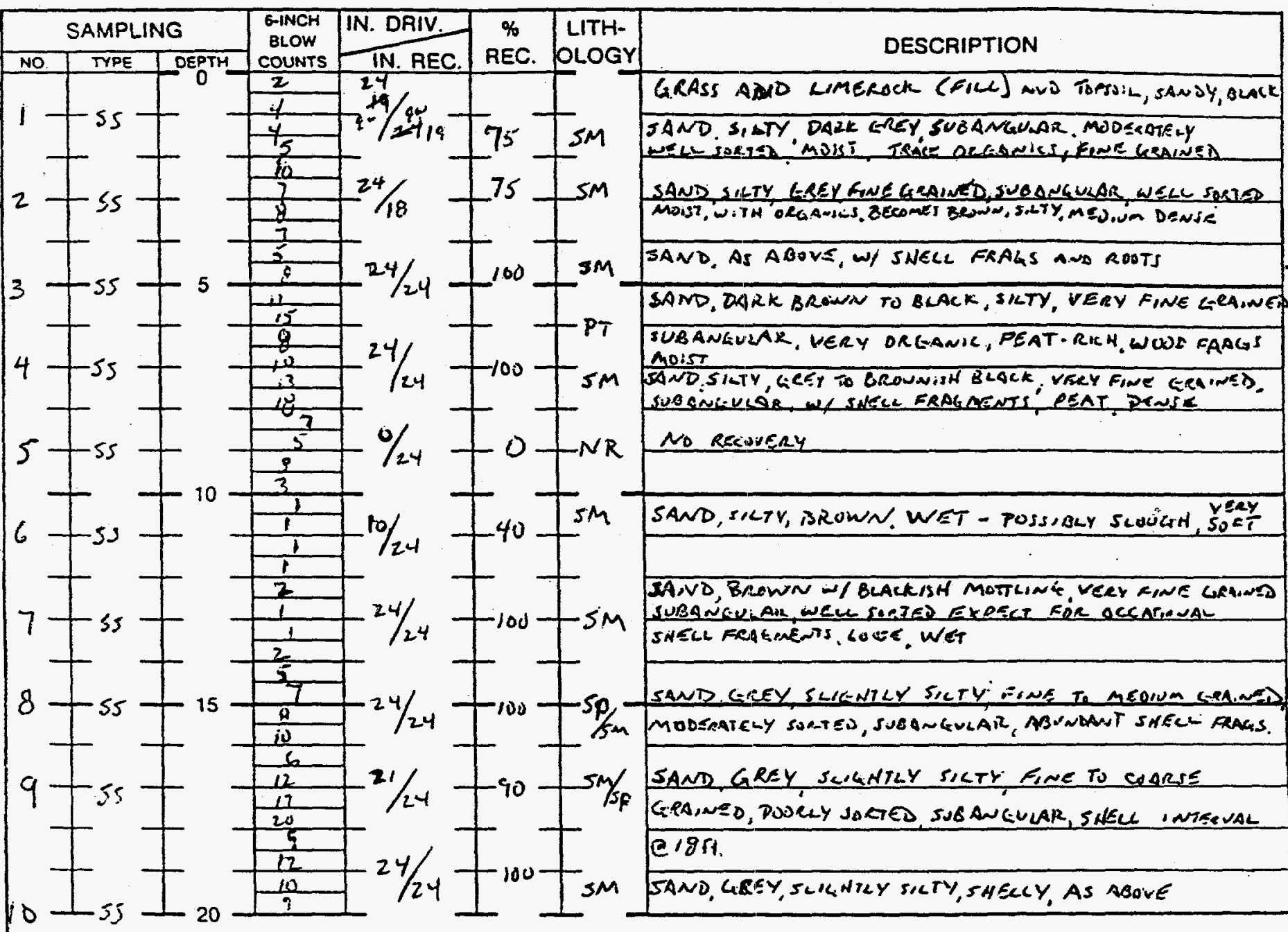

Samples Submitted for Laboratory Tests

\begin{tabular}{|l|l|l|}
\hline & & \\
\hline & & \\
\hline & & \\
\hline
\end{tabular}

NOTE: Standard Penelration Resistance is Sum of Blows for 2nd $-6^{\prime \prime}$ and 3ro - 6" to Drive 1-3/8" I.D., 2" O.D. Split Barrel Sampler with 140 pound hammer falling 30 inches 


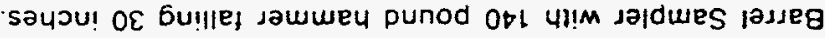

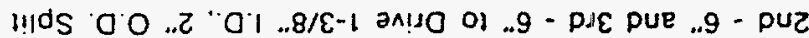

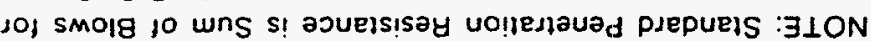

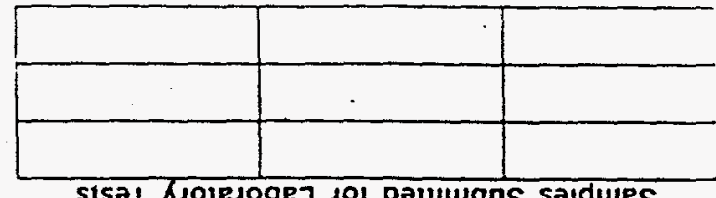

sisal hojesoge? 201 pantuqns saidues

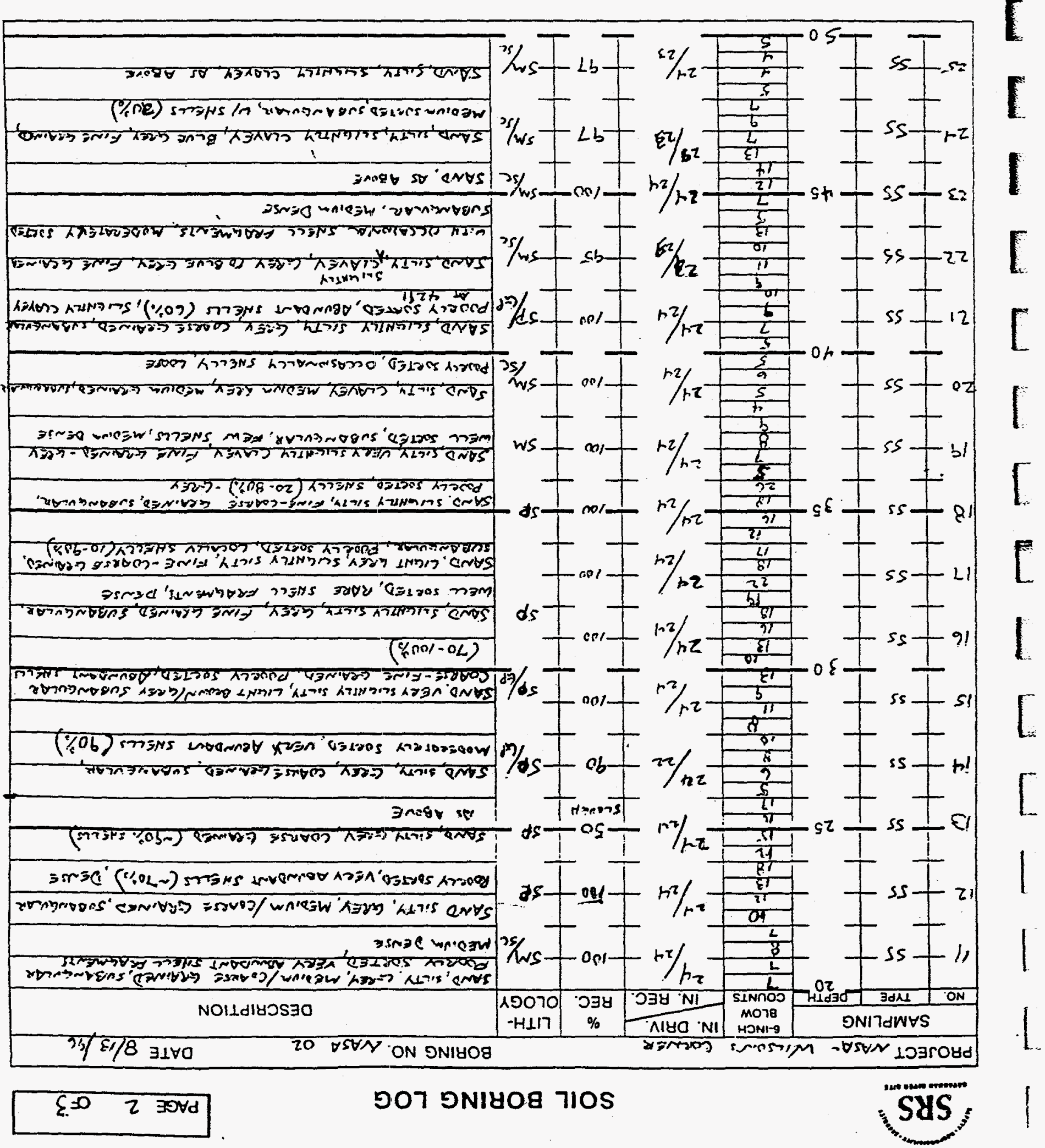

$(2601 \cdot \times 9) \quad 2 x \cdot \rightarrow 1$ yso 
BORING NO. NASA-DZ

DATE $8 / 1+1 / 96$

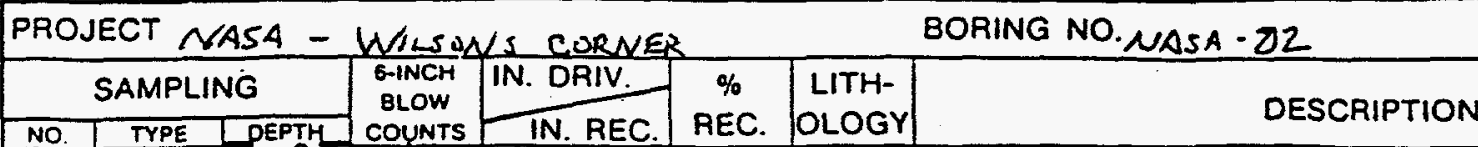

STM. SAND, SICTY, SWI4ATLY CLAYEY, BLCE GREY, SUSANGUUAR,
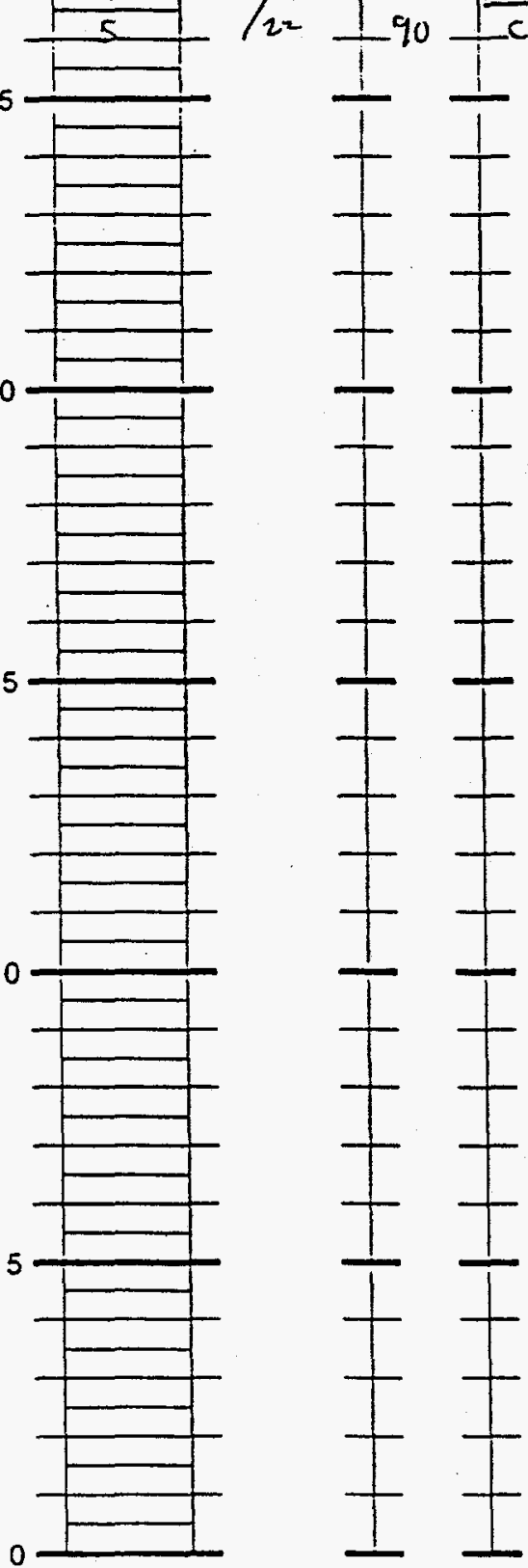

NOTE: Standard Penetration Resistance is Sum of Blows for 2nd $-6^{\prime \prime}$ and 3rd $-6{ }^{*}$ to Drive 1-3/8" 1.D.. 2" O.D. Split Barrel Sampler with 140 pound hammer falling 30 inches. 
sausu! of 6u!lle; sawmey punod opl y!!m sajdures passeg

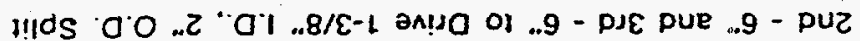
sof SMolg to wns s! asuejs!say vo!neıəuad psepuejs : $\exists 10 \mathrm{~N}$

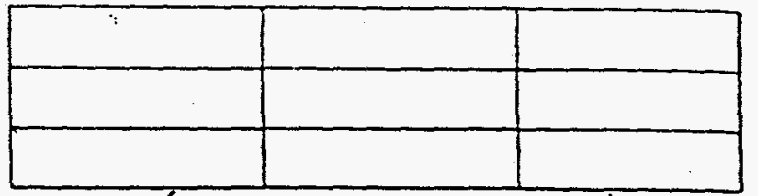

sisol kiojesoqe7 dol paltugns soldues

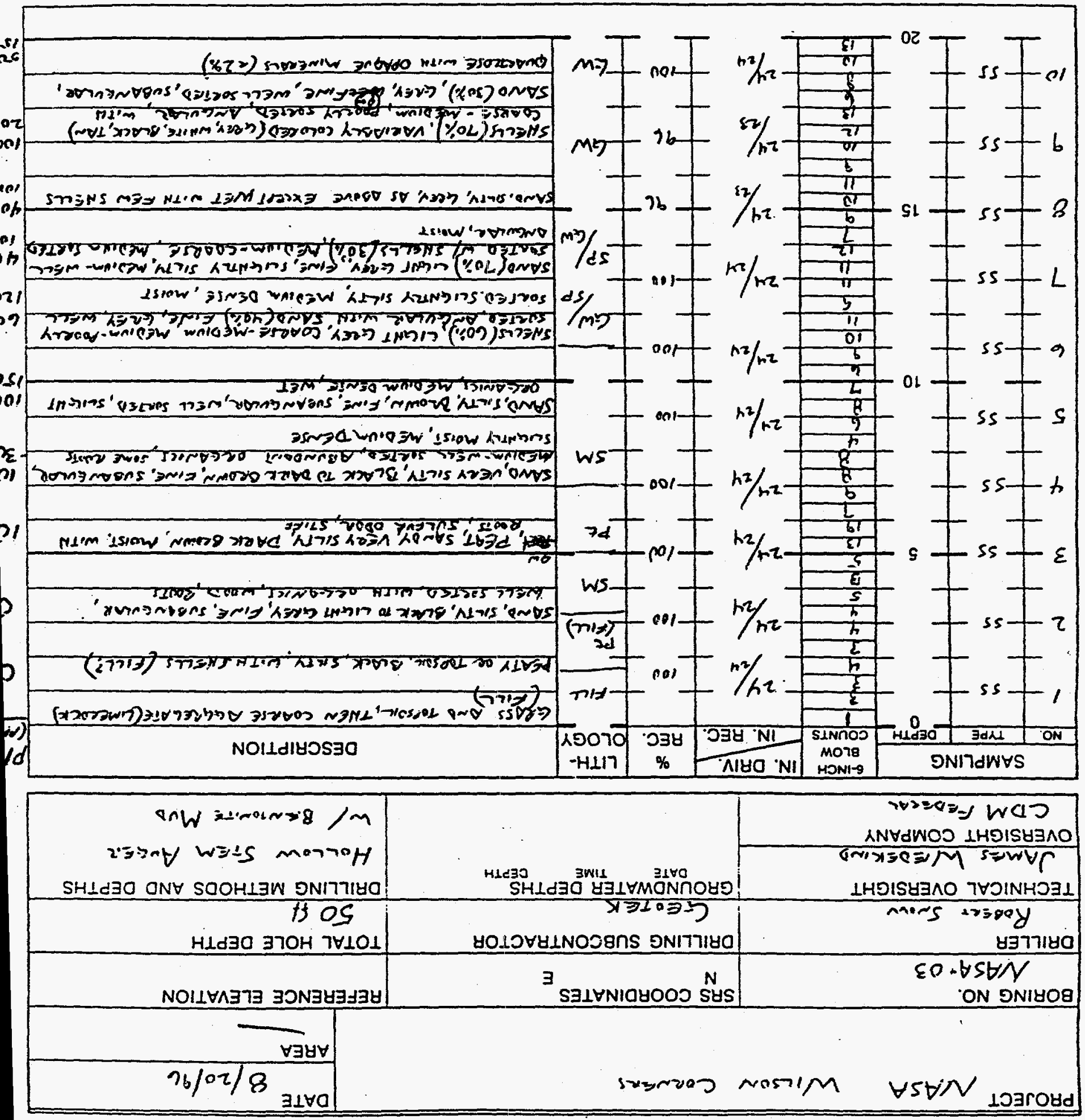


'sayou! of 6u!lle; samwey punod ODL 4l!M dajdwes paseg

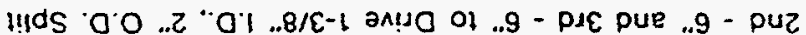
so) smols to uns s! asue!s!say uo!jejlauad puepueis : $\exists 10 \mathrm{~N}$

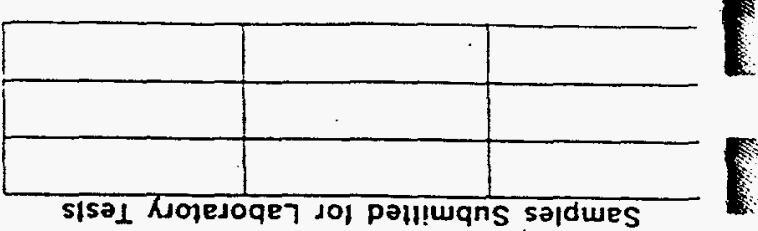

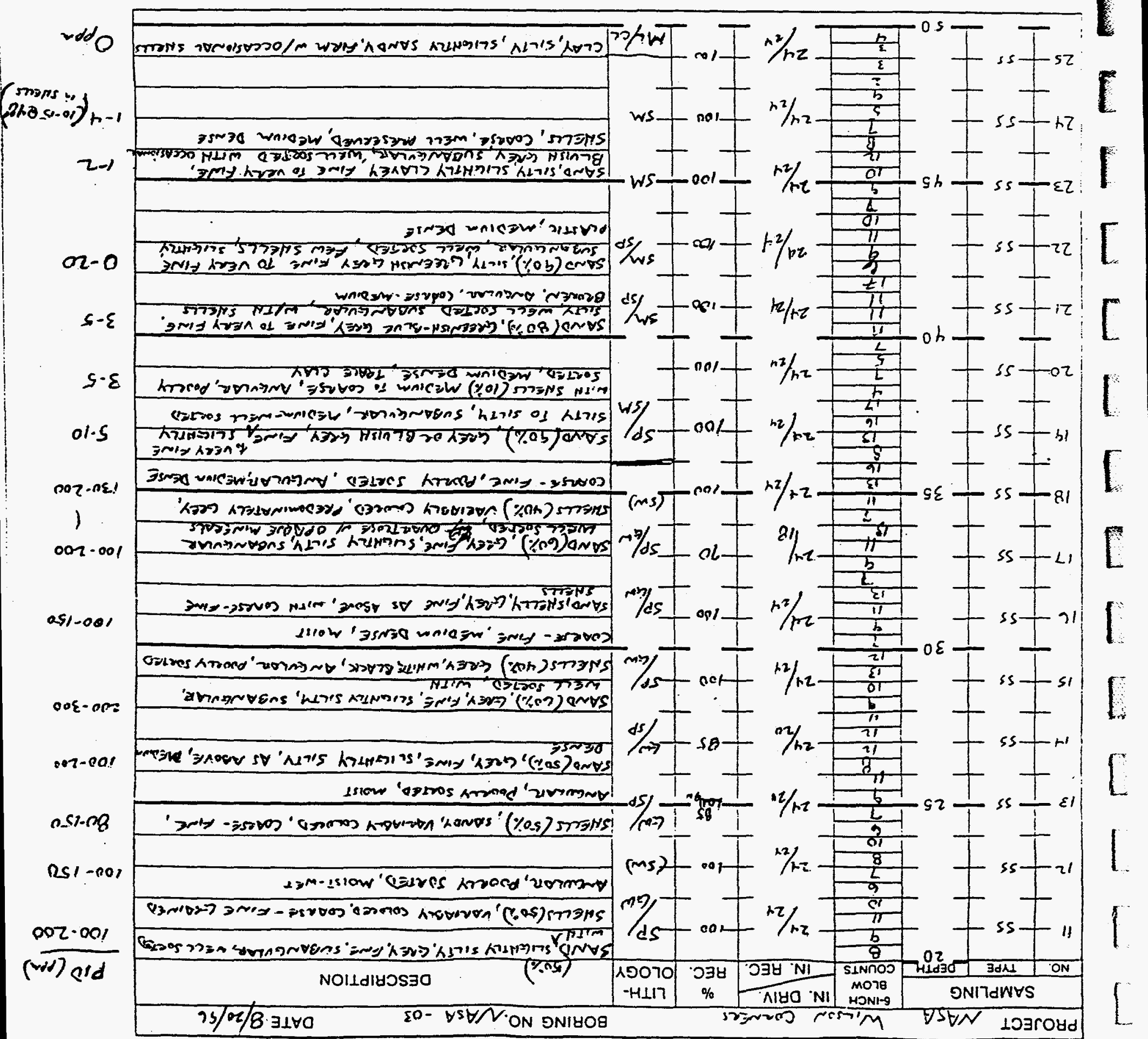




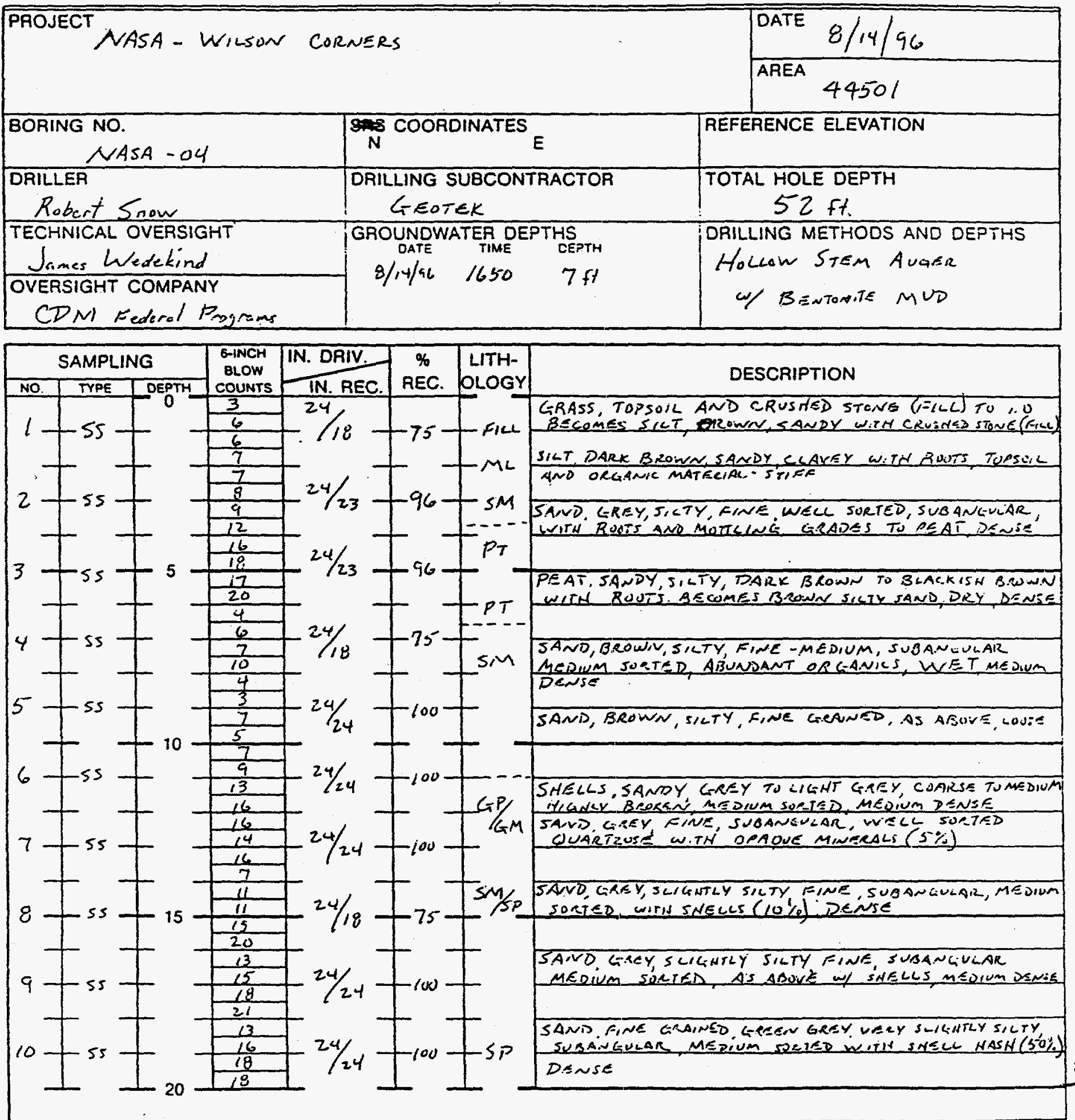

Samples Submitled tor Laboratory Tests

\begin{tabular}{|l|l|l|}
\hline & & \\
\hline & & \\
\hline & & \\
\hline
\end{tabular}

NOTE: Slandard Penetration Resistance is Sum of Blows for 2nd $-6 "$ and 3rd - 6" to Drive 1-3/8" 1.D. 2" O.D. Split Barrel Sampler with 140 pound hammer falling 30 inches. 
sayวu! of Gu!lle! saumey punod otl ul!m saldwes lasseg

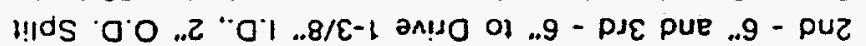

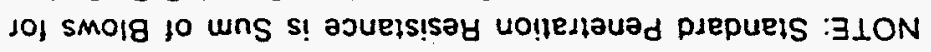

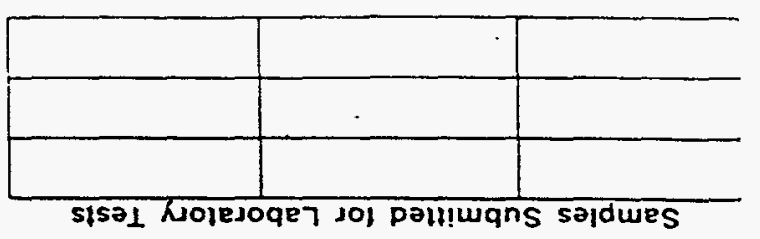

sisal kulejogeר 101 poll!wans sejdues

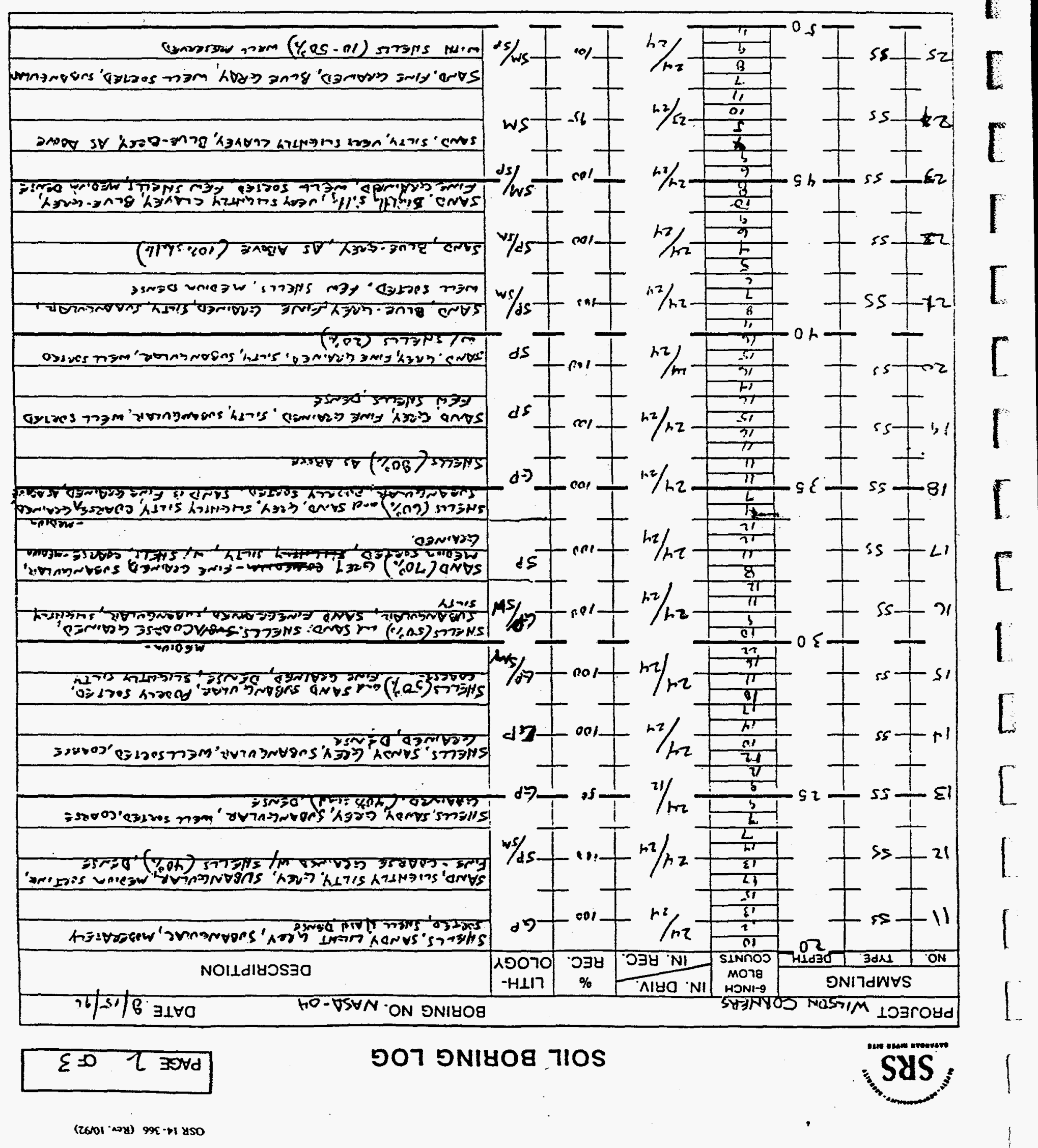


sauoul of bullef sawwey punod opl ul! sajdwes lasseg

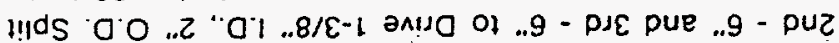

دo) SMOIG 10 uns s! әJueis!sar uo!lestauad psepuels : $310 \mathrm{~N}$

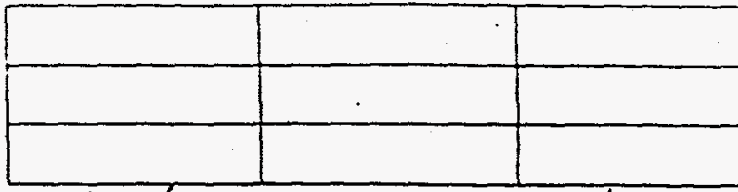

sisal KJojejoqe7 dol poll!wans soldues

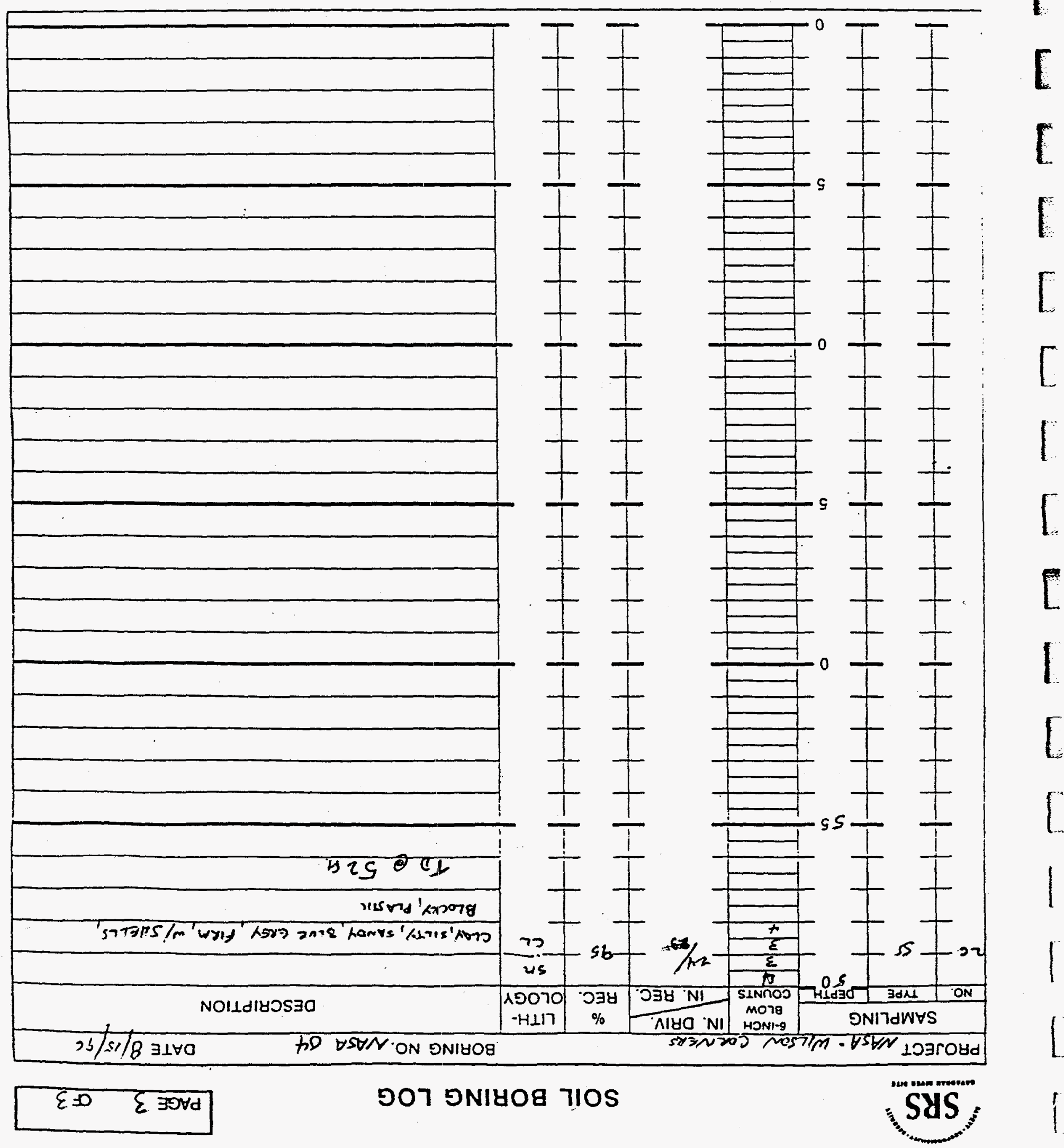




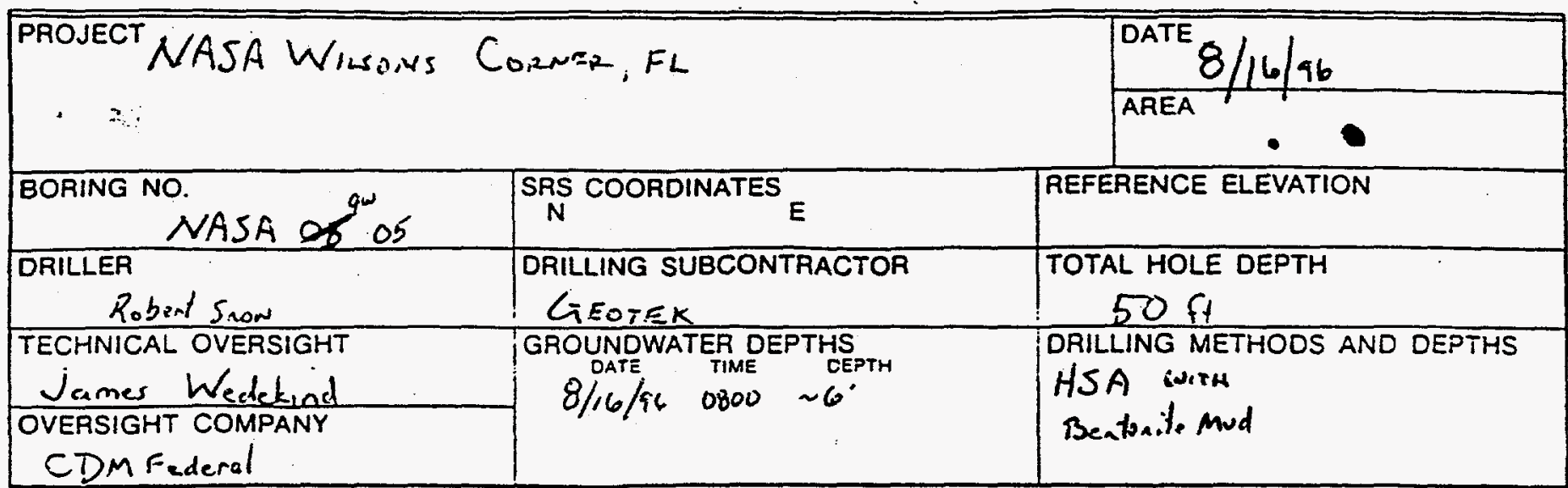

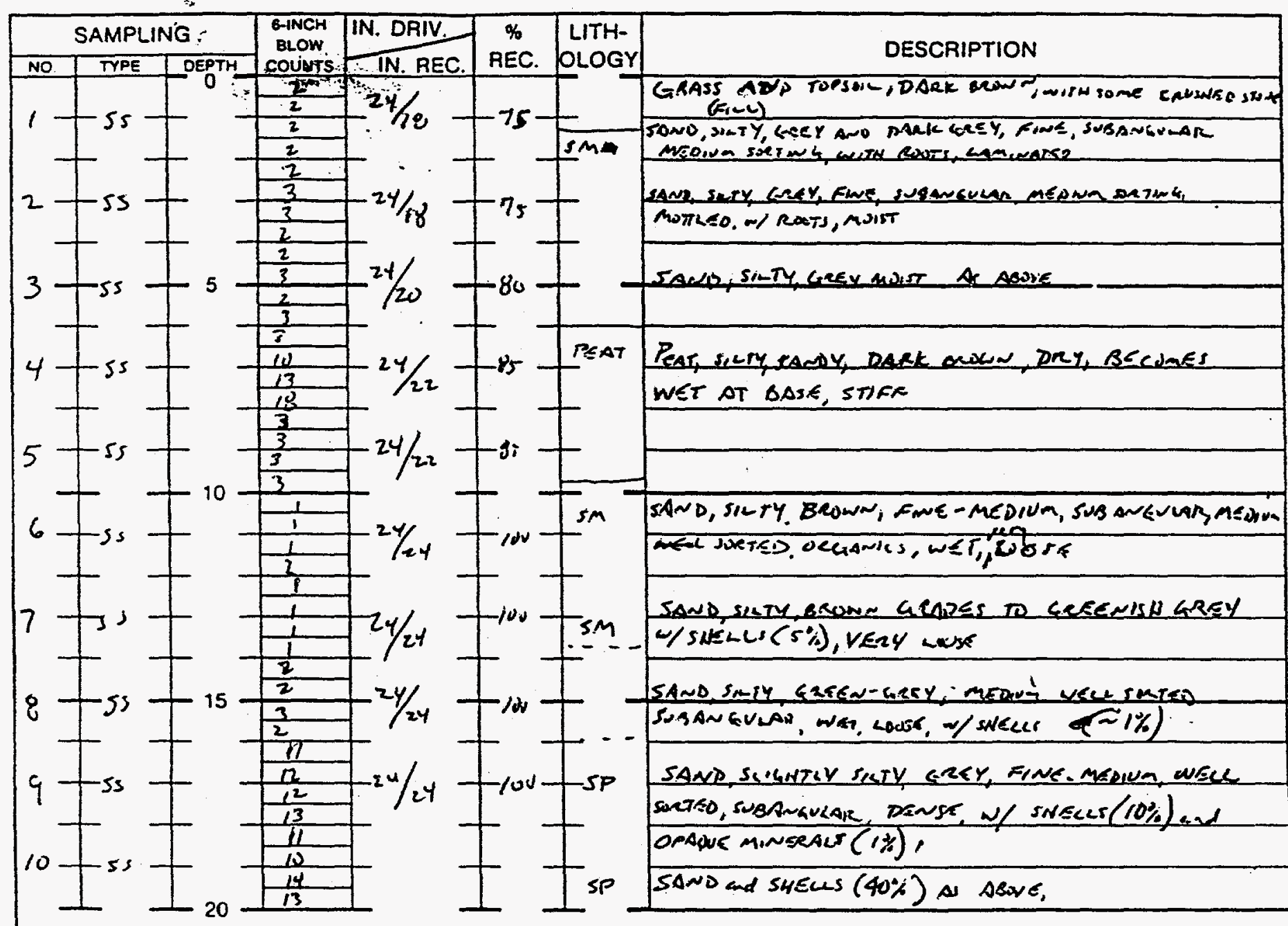

Samples Submitted for Laboratory Tesis

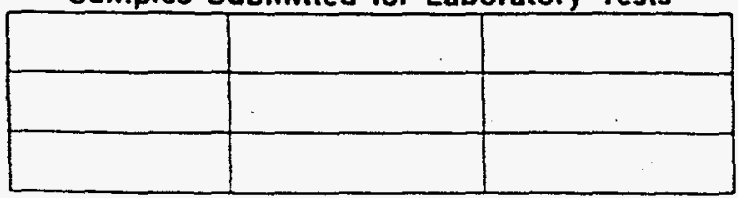

NOTE: Standard Penetration Resistance is Sum of Blows for 2nd - 6" and 3rd - 6" to Drive 1-3/8" 1.D. 2" O.D. Split Barrel Sampler with 140 pound hammer falling 30 inches 


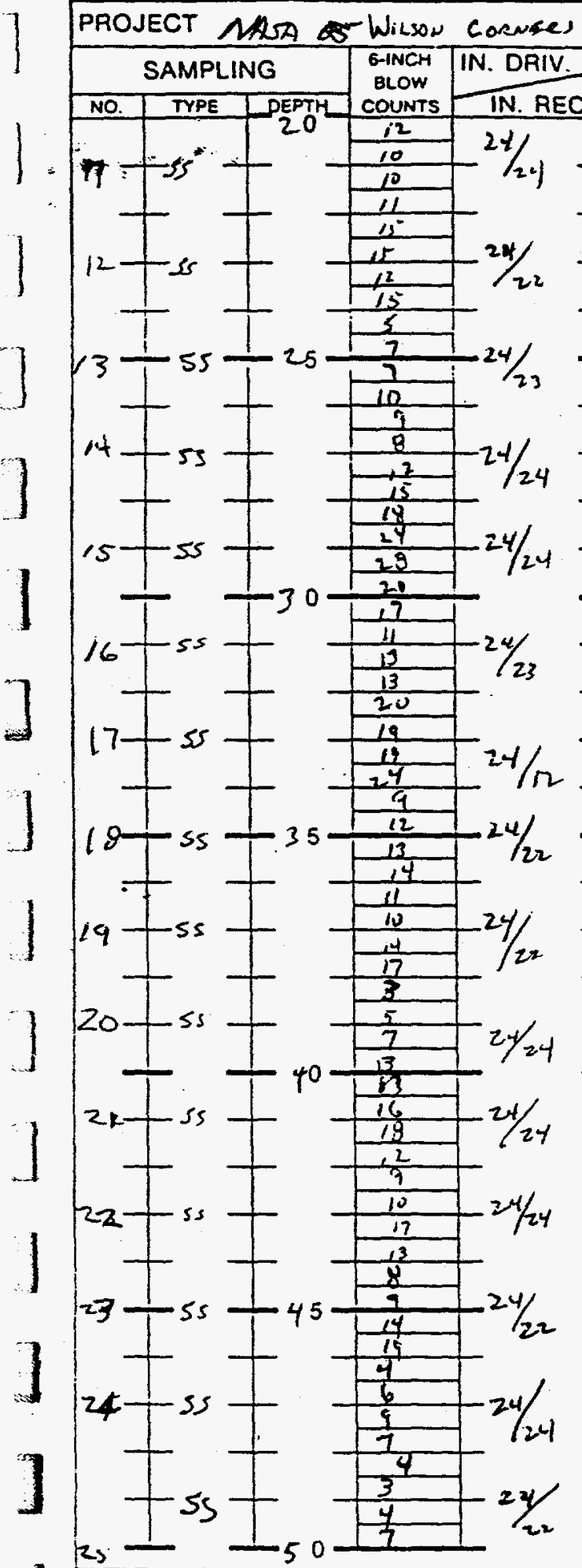

1

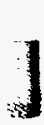

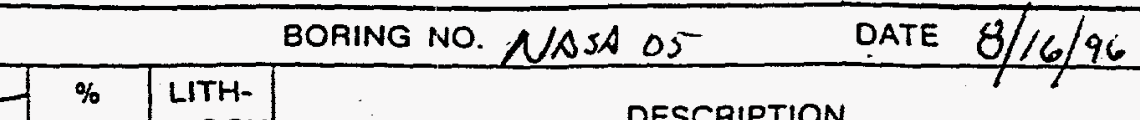

DESCRIPTION

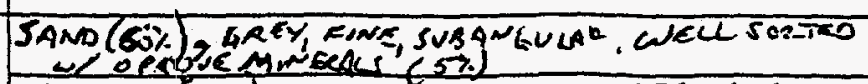

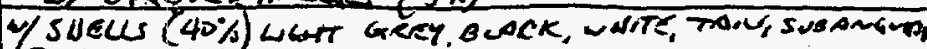
coucse-mediva

SAND (70

SUSE (30) AS ABOY

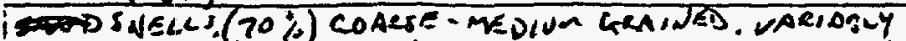

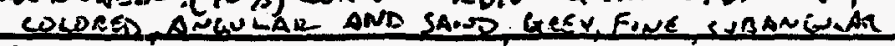
Domarese Qunetzose

SNELCS $(90 \%)$ AS. ASOVE

SANA ( $10 \%$ ) AS $A B$ BVE

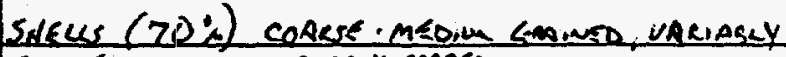

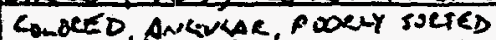

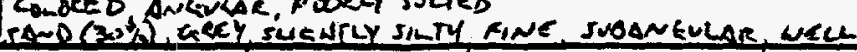

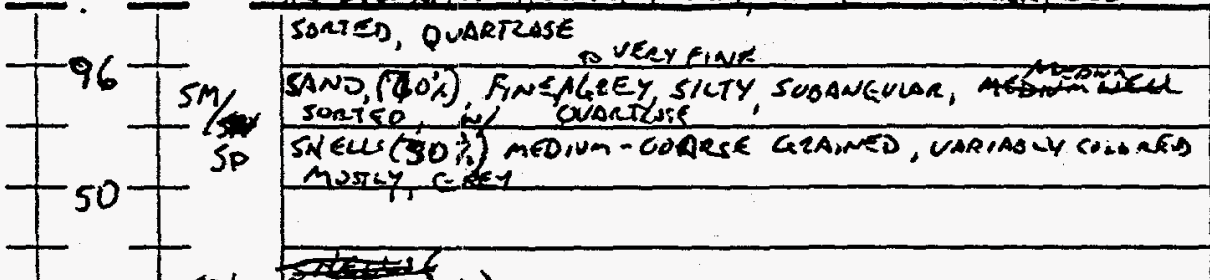

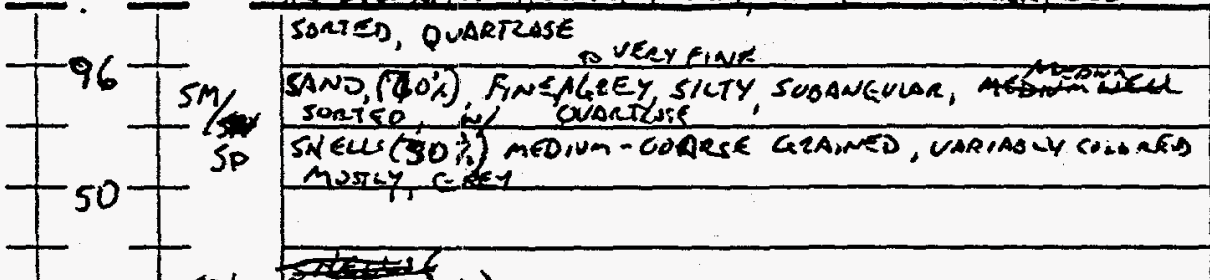

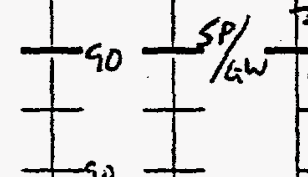

5AND (60\%). Fine ciand ceay Gine susankuiar

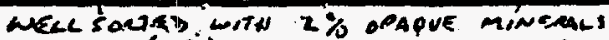

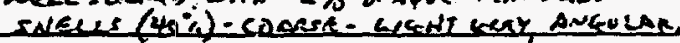

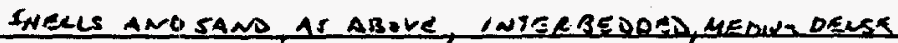

SAND, SWLWTHY SILTY GREY, TO BLUE GLEY, FINE, SUBANENLAR,

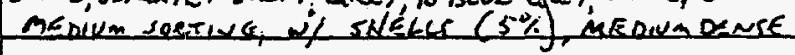

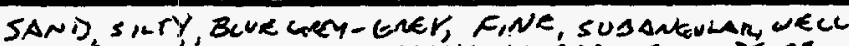

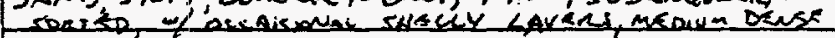

SAND SNTY WERY SWANTLY CLAVEY FINE - VERY FIYE

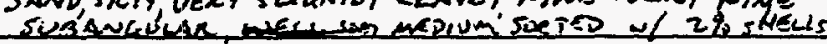
BLwe-are:

SAMO, SILTY, BLUE GREY, AS ABOHE

SANO SILTY SWGHTLY CLAYEY, FINE TO VERY FINE,

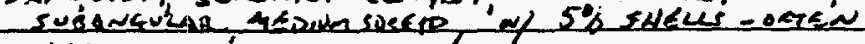

CLAY, JMTY, SANDY, FVITN SANDY LAMNADE unaroken

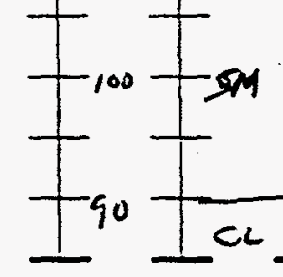

\section{TD@50Ft.}

Samples Submitted for Laboratory Tests
NOTE: Standard Penetration Resistance is Sum of Blows for 2nd - 6" and 3rd - 6" to Drive 1-3/8" 1.D., 2" O.D. Split Barrel Sampler with 140 pound hammer falling 30 inches. 


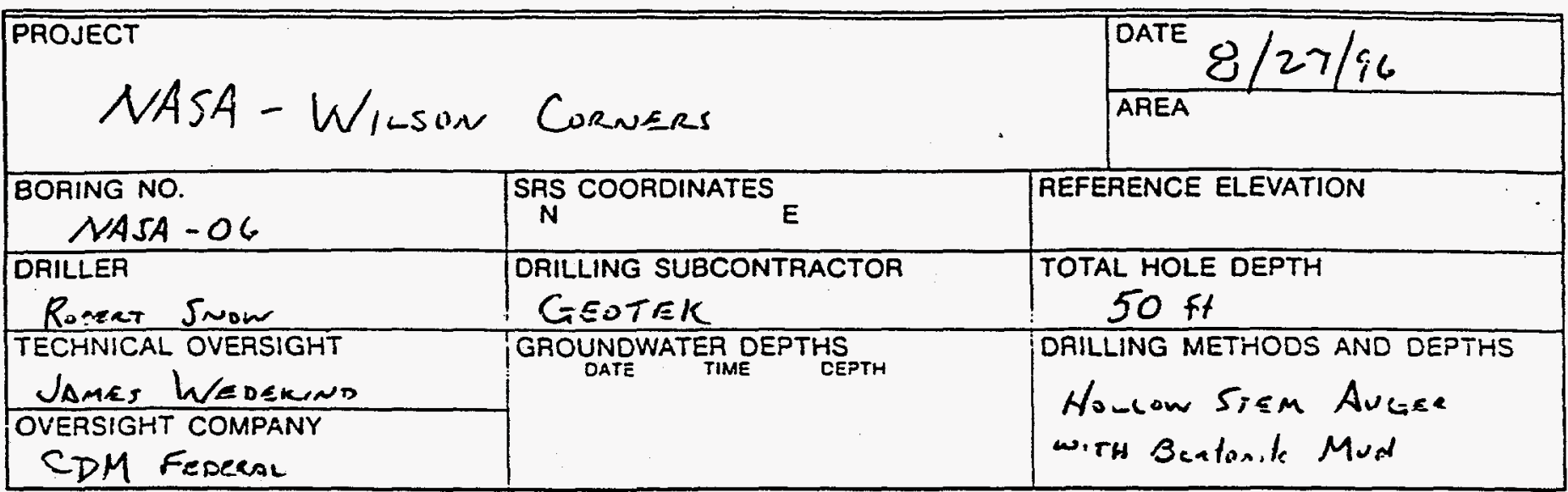

LITHE
OLOGY DESCRIPTION

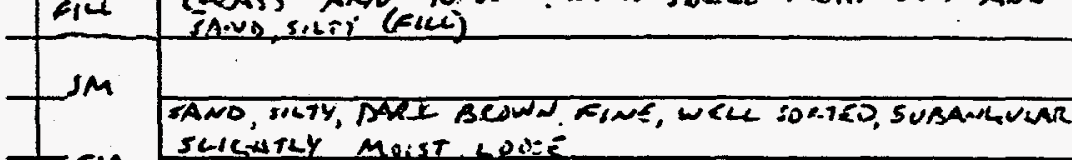

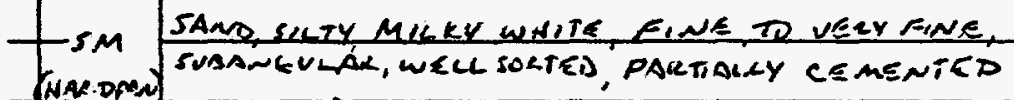
(WAR:DMN) (HADDRAN), MUIST, MED,JM DENSE 


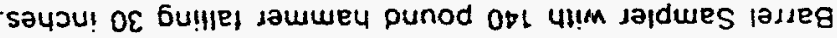
unds $0.0 .2 \cdot 0 . .8 / \varepsilon-1$ anilo ol ... - p/c pue ..9- puz sof smolg to uns s! әJuels!say uollenauad psepueis : $\exists 10 \mathrm{~N}$

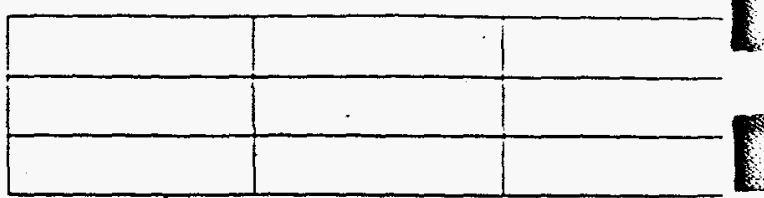

sisal Kolesoqe7 jol palt!wqnS səjdues

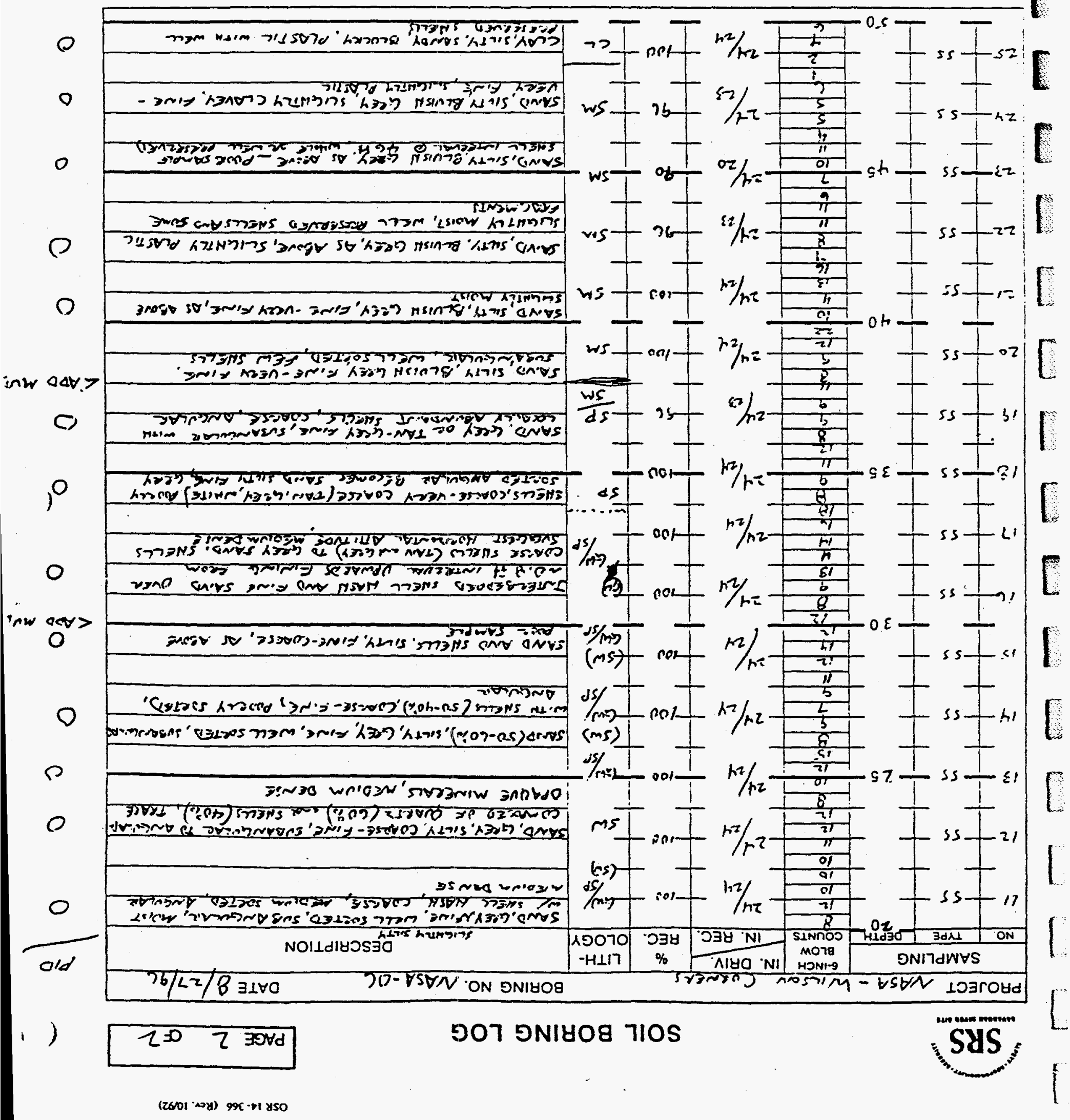




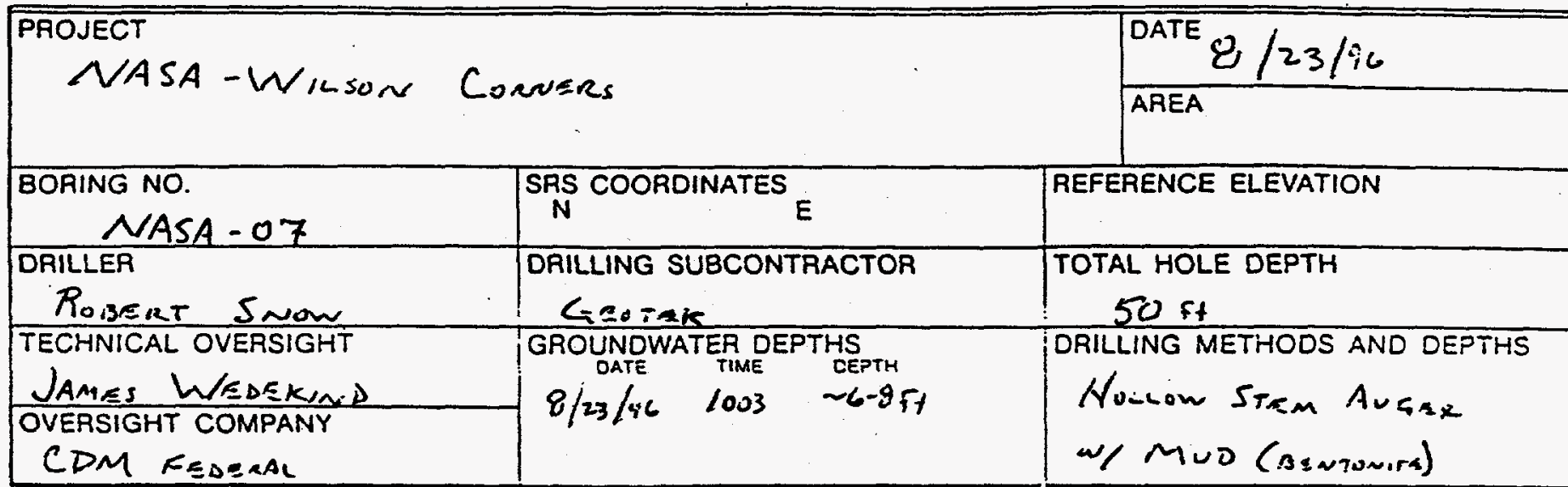

1

1

1

3

3

3

3

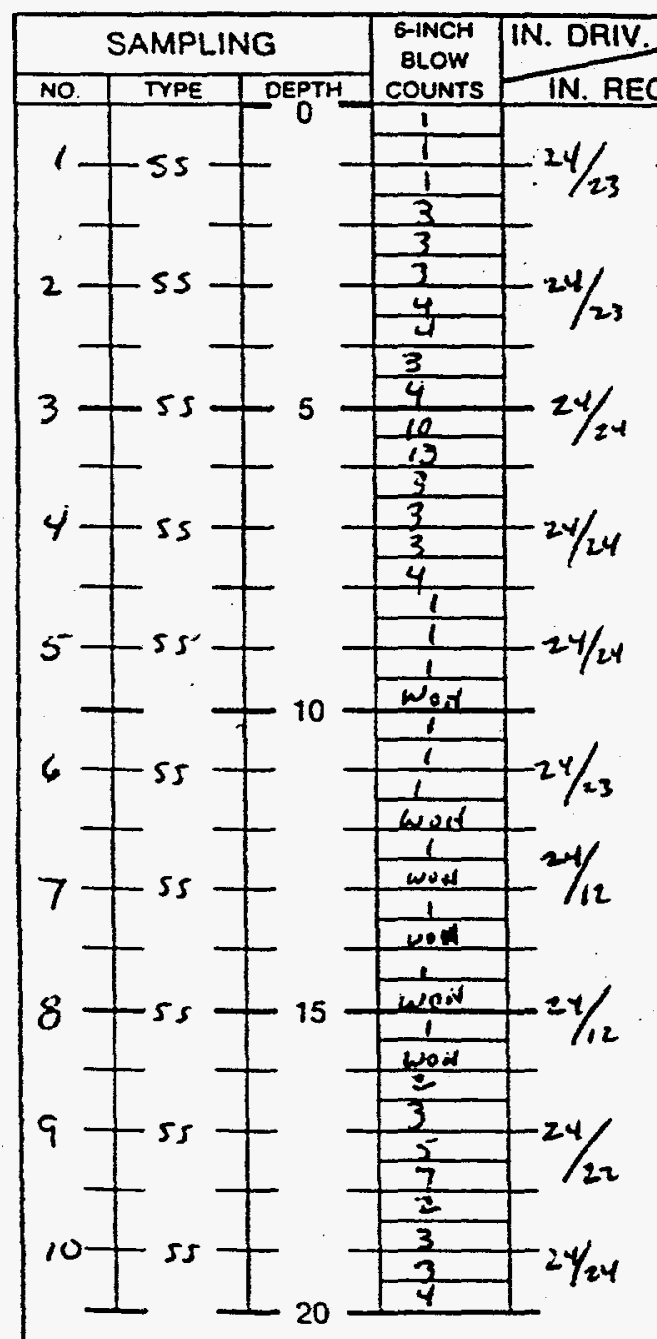

DESCRIPTION

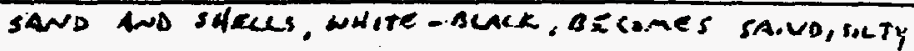

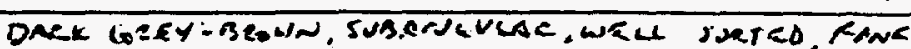

SANO LGNGREY SULTY EINE THENWA-wKLL SURTED. MoIst, w/ ROOTI, TEACE DEL QNMS

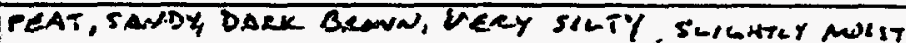

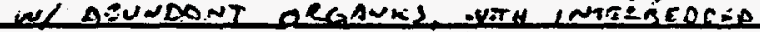

SAIVD, ILTY, LCWT GAEY, W/ ROCIS, STIFF

SA.ND BEUWN, VECY SALY FING, IUS ANGWWX, WELL roveras, Moist, wouse swafve osce

SAND, WGWT BROWN, SOLTY, FINE, SUBANGULAR, WELL

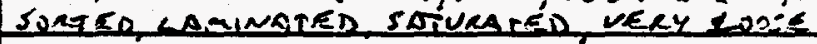

SANB, CILAT BROWN, JWTV, FINA, AS AONE, SWLANT Decienk, SATVRATED, VECY LATCK

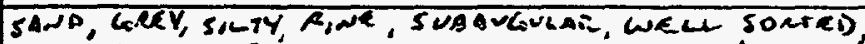

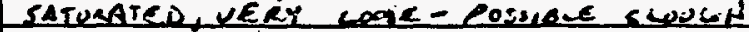

SARO,A: A.3OVE - POOR SAMOLE

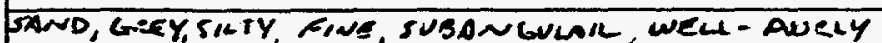

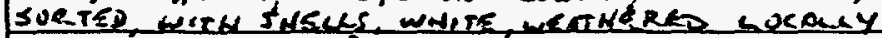
ASUnBA $(2-25 \%)$

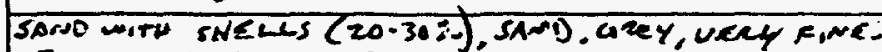
Fene, sices cuoere-enkbum
Samples Submitted lor Laboratory Tests

\begin{tabular}{|l|l|l|}
\hline & & \\
\hline & & \\
\hline & & \\
\hline
\end{tabular}

NOTE: Standard Penetration Resistance is Sum of Blows for 2nd - 6" and 3rd - 6" to Drive 1-3/8" 1.D. 2" O.D. Split Barrel Sampler with 140 pound hammer falling 30 inches. 
-sayou! of bu!lle sowmey punod orl 4!!M soldwes jasseg

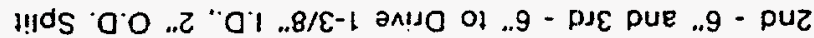

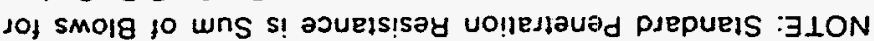

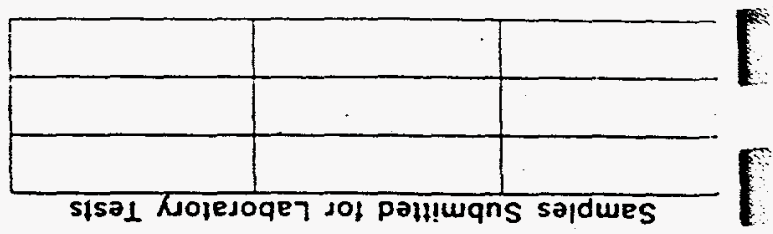

inimas

prow aid

$0 z>$

$a z-0)$

or

)

0

O

0

0

O

Q1/d

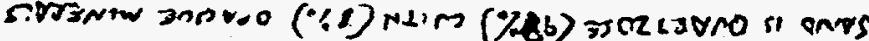

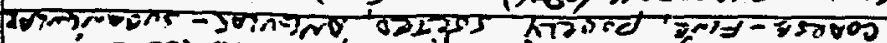

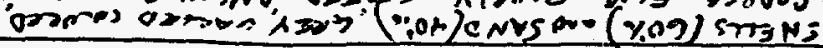

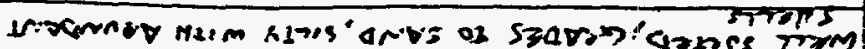

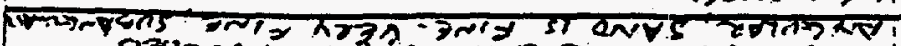

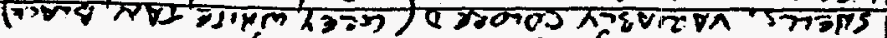

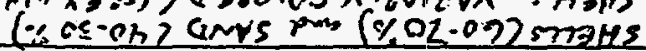

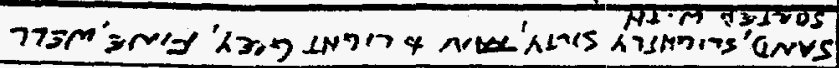

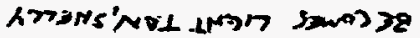

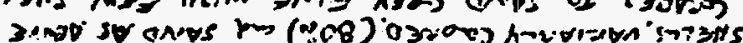

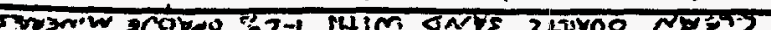

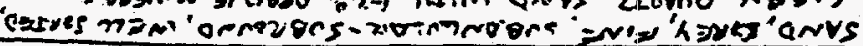

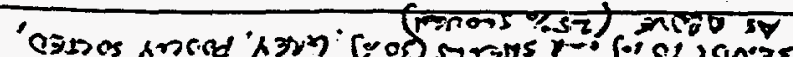

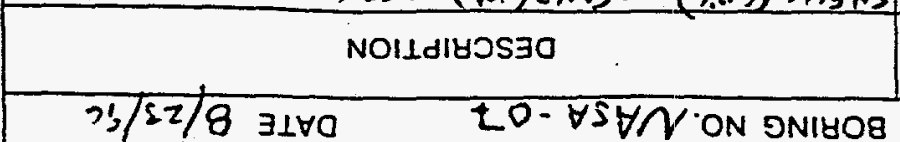

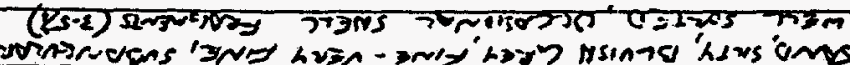

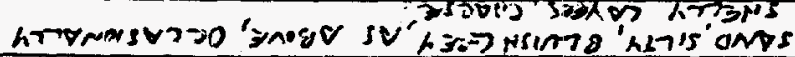

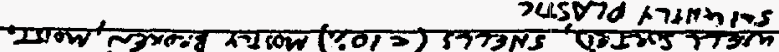

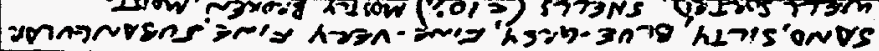

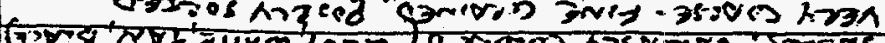
35 ines $\% 13$ stoms zwos.

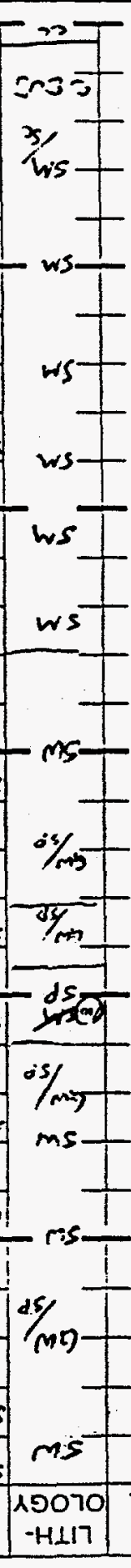

$n o 1+m / m_{2}$ $\left[\begin{array}{l}0 \\ 0_{b}\end{array}\right]$

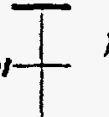

$\mathrm{m} / \mathrm{m}$

\begin{tabular}{|r|r|}
\hline \multicolumn{1}{|c|}{} \\
\hline \multicolumn{1}{|c|}{} \\
\hline
\end{tabular}

$22 / n=$

$r^{2} / \mathrm{re}$

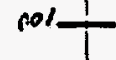

$22 / 2$

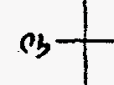

\section{0
$-H 117$}

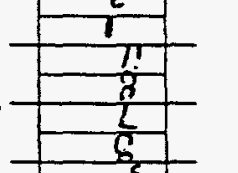

$f_{s s} T_{s=}$

$\pi / n$

L

$\frac{\xi}{\varepsilon}$

st

$+5 s+t_{2}$

$22 / 4=$

है।

$\frac{2}{\frac{2}{2}}$

$\frac{9}{\frac{9}{9}}$

$+1$ (0)

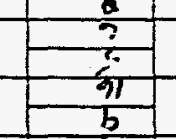

$t_{55}+:=$

$\varepsilon 2$

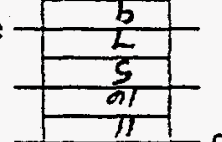

$n+\frac{1}{1} \pi$

$\varepsilon 2 / 42$

$\frac{-5}{\frac{b}{5}}$

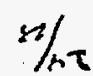

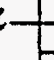

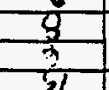

is

ood $t \mathrm{hz} / \mathrm{h}=$

$h / h=\frac{\frac{\delta}{5}}{\frac{2}{2}}$

$r 2 /$

$/=$

$\frac{2}{21}$<smiles>[H]</smiles>

जो

$\mathrm{h} / \mathrm{ne}$

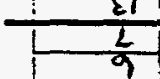

$\mathrm{kr} / \mathrm{n}$

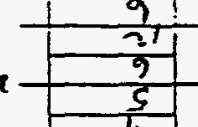

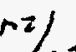

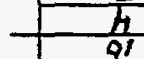

क्

$t_{s s+1}$

$\frac{f}{T}{ }^{1}={ }^{2}$

1

$\Gamma$

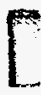<smiles>C1CCCCC1</smiles>

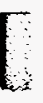

I

I

1

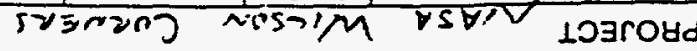

DO7 DNIYOA 7IOS

SyS
$2=0 \quad z$ 3ord

(z6501-ay) $99 x-1$ yso 
PROJECT

$$
\text { NASA-Qg Wilson coeners }
$$

$$
\text { DATE } 8 / 29 / 96
$$

BORING NO.

\section{DRILLER}

$$
\text { N/9SA - } 09
$$

Robiot Snow TECHNICAL OVERSIGHT Jame, Widelind OVERSIGHT COMPANY CDM Feder 1

SRS COORDINATES

DRILLING SUBCONTRACTOR

GEOTEK

GROUNDWATER DEPTHS

CEPTH

\section{TOTAL HOLE DEPTH}

50 A

DRILLING METHODS AND DEPTHS Hollow 5TEM AviER

GCArs and SANO, Beawn, SeLTY

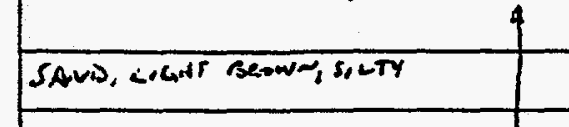

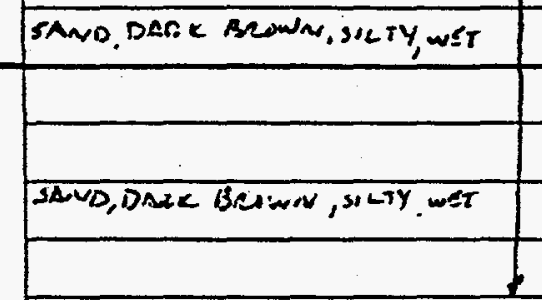

SANM, SILTY GEEY 3ECJMES LIGATER GESY FINE, WELL

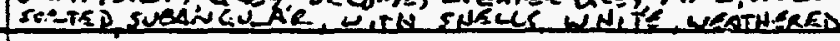
a 2 - in INTERUAL $Q \sim 1551$.

Samples Submitted for Laboratory Tests

\begin{tabular}{|l|l|l|}
\hline & & \\
\hline & & \\
\hline & & \\
\hline
\end{tabular}

NOTE: Standard Penetration Resistance is Sum of Blows for 2nd - 6" and 3rd - 6" to Drive 1-3/8" 1.D., 2" O.D. Split Barrel Sampler with 140 pound hammer falling 30 inches. 
sayou! of 6u!lle samwey punod ot Ll!m japdwes lasseg

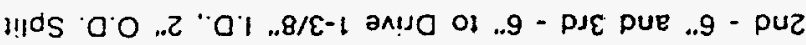

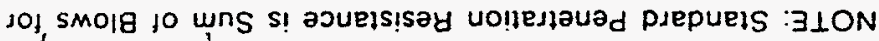

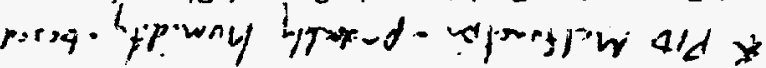

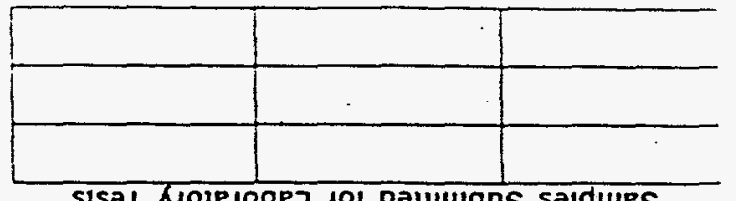

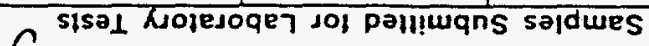

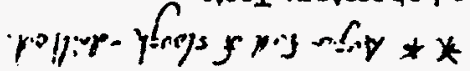

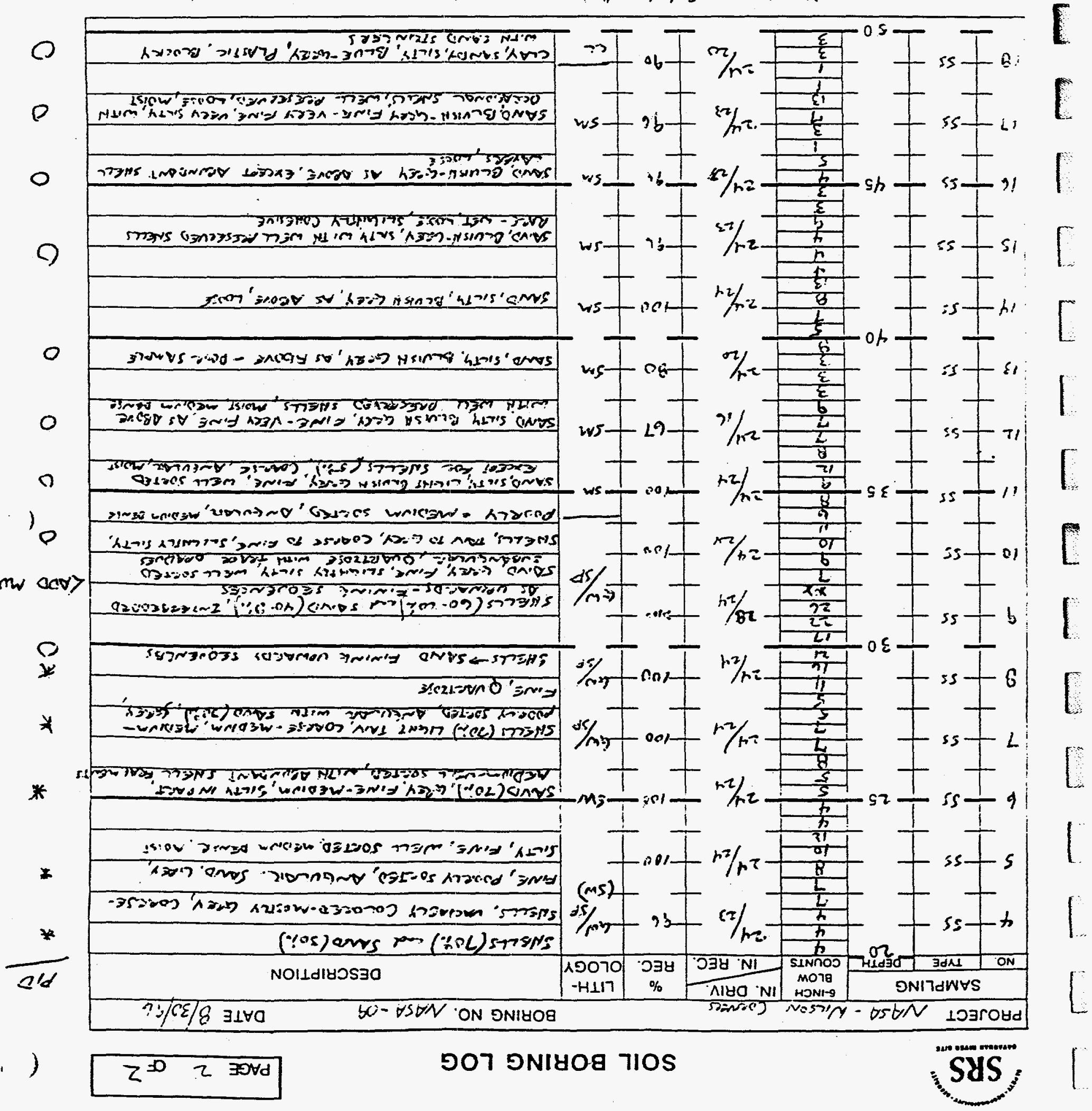




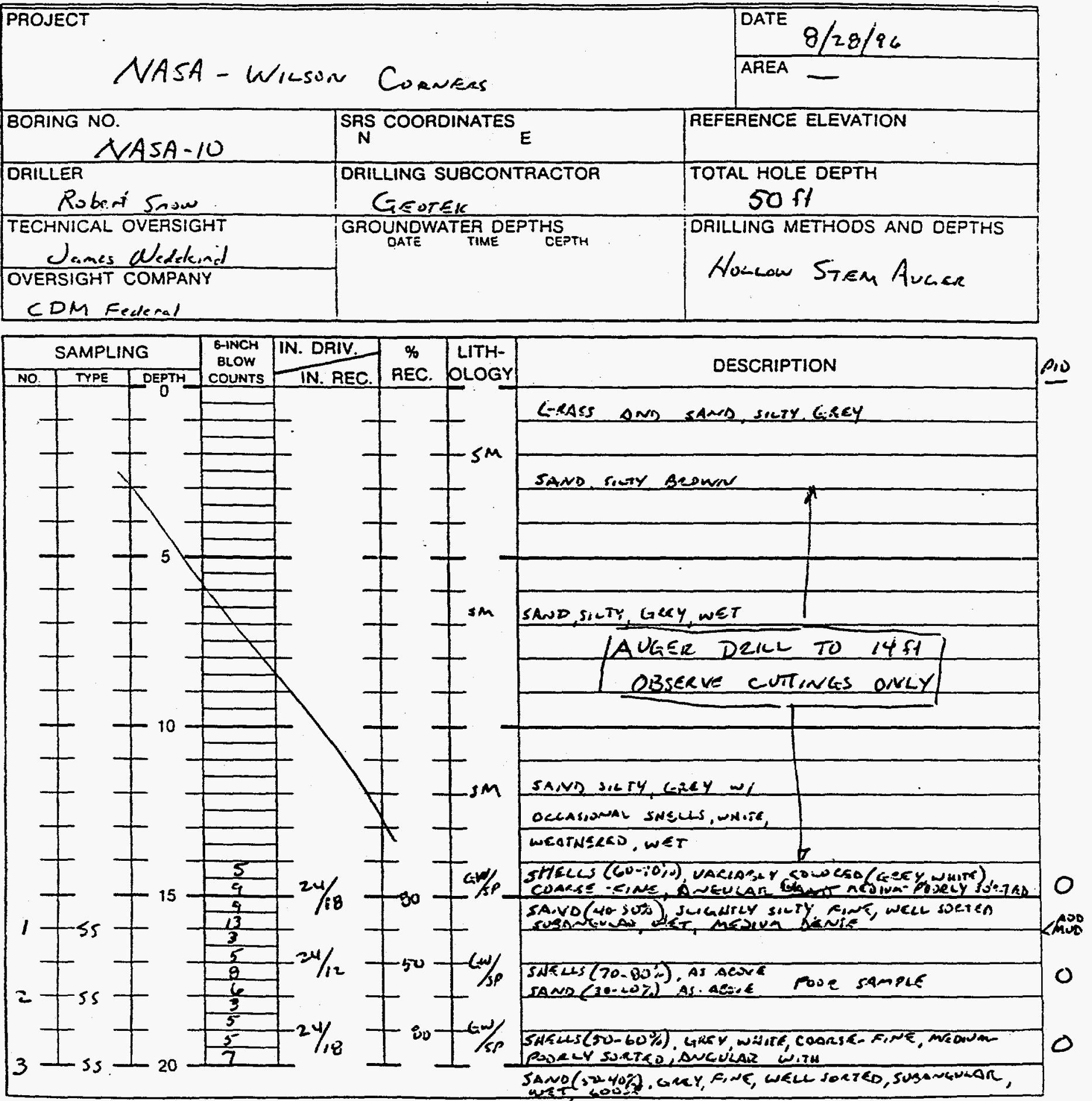

Samples Submitted for Laboratory Tests

\begin{tabular}{|l|l|l|}
\hline & & \\
\hline & & \\
\hline & & \\
\hline
\end{tabular}

NOTE: Standard Penetration Resistance is Sum of Blows for 2nd - 6" and 3rd - 6" to Drive 1-3/8" I.D. 2" O.0. Split Barrel Sampler with 140 pound hammer falting 30 inches 


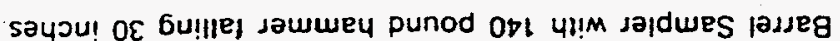

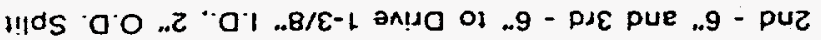

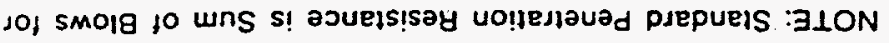

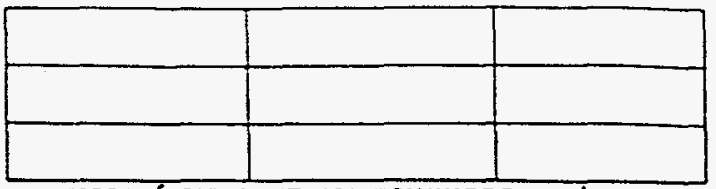

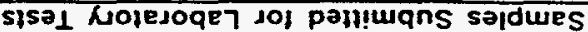

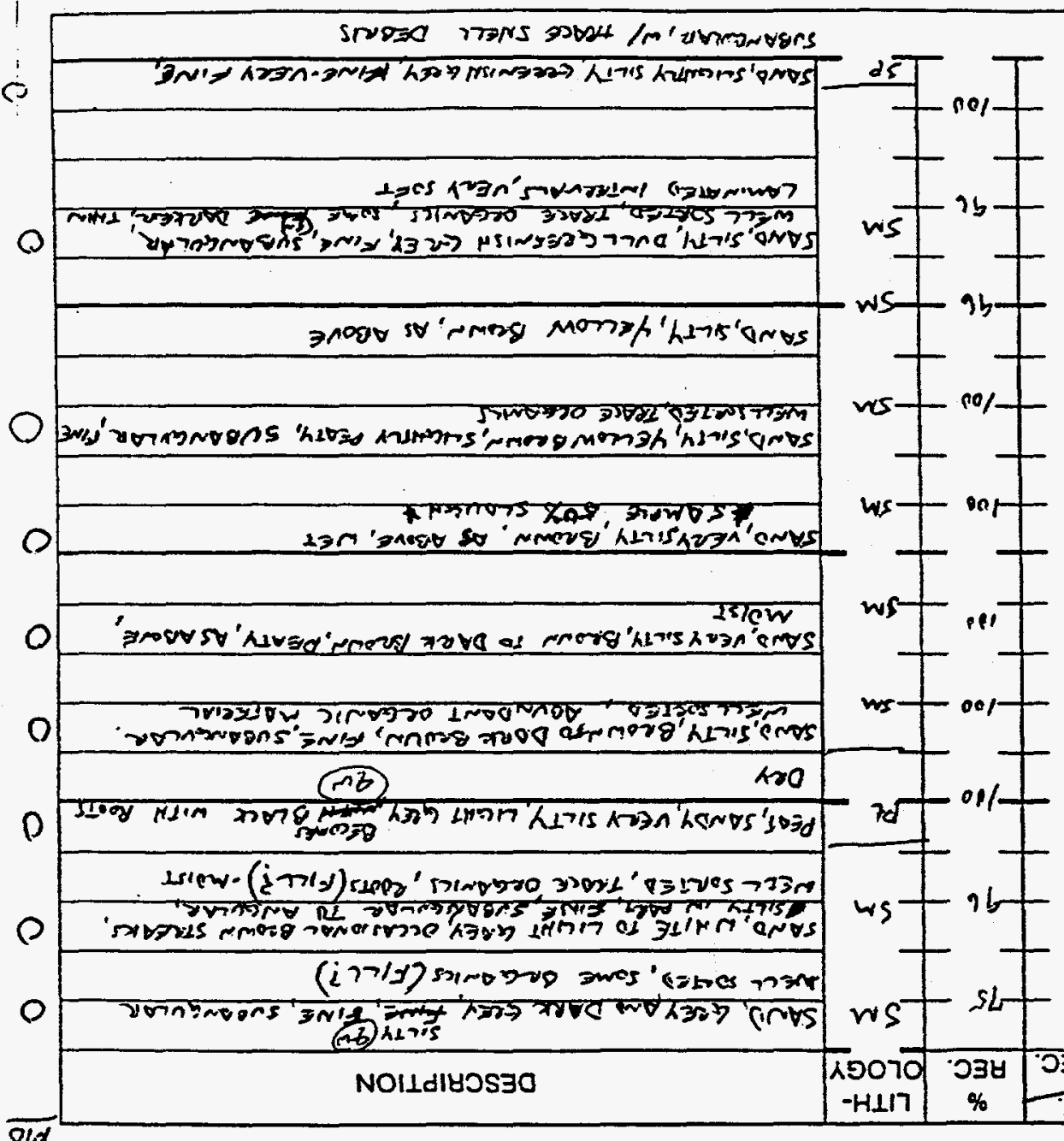

$\nabla /$

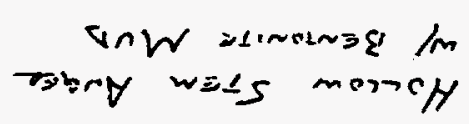

SHIdヨO ONY SOOHIBW ONIרาIYO

$+525$

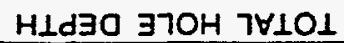

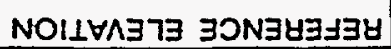

$$
9 b / b 1 / \beta_{\exists \perp \forall 0}^{\forall \ni y t}
$$

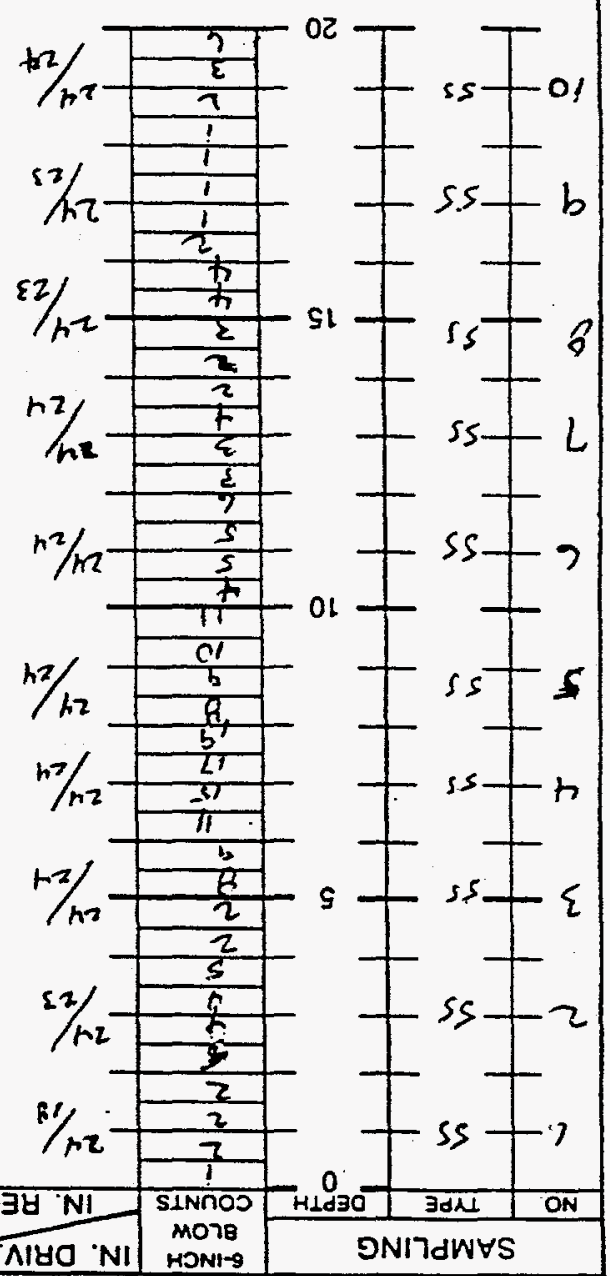

rosegyy woJ ANYdWOO 1 HSISH3^O

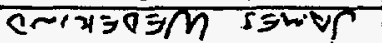

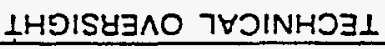
mor's $10 \mathrm{~s}$. ㅂำเช8

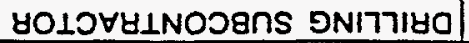
$\exists$ SILVNIOYOOD $\stackrel{N}{\text { SUS }}$ $11-\theta \Delta \theta /$ ON ONIBOA saznzog nosaim - HSHN 103 rovd

\section{$\sum 50 \cdot 1$ 3DAd


PROJECT NASA WILSJN CuEver,

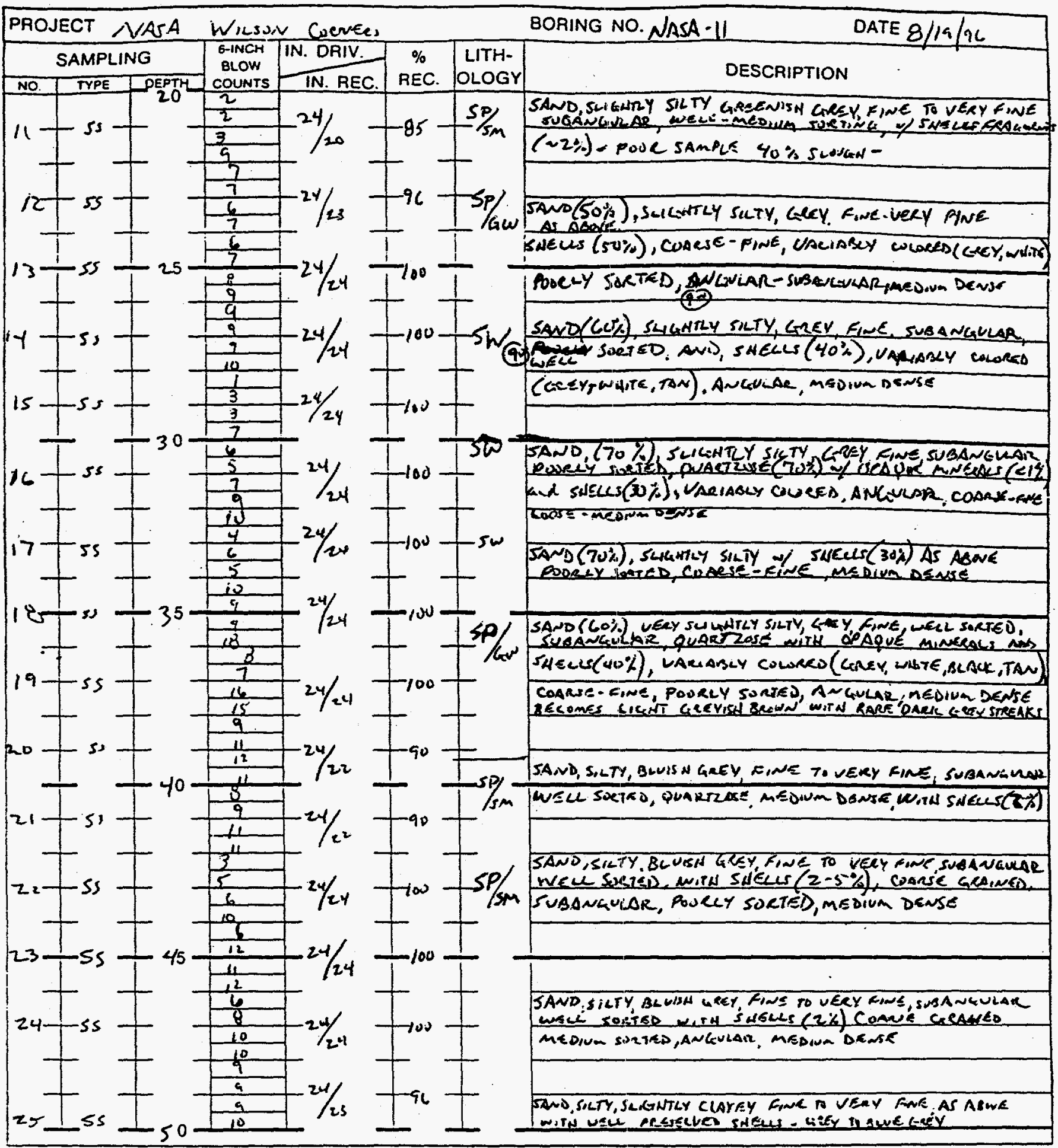


SRS anposest enve uns
SOIL BORING LOG

PROJECT NASA W. LSON CucNeLs \begin{tabular}{|c|c|c|c|c|}
\hline \multicolumn{3}{|c|}{ SAMPLING } & $\begin{array}{c}\text { 6-INCH } \\
\text { BLOW } \\
\text { CLOUNTS }\end{array}$ & IN. DRIV. REC. \\
\hline NO. & TYPE & DPEPTH \\
\hline & & &
\end{tabular}

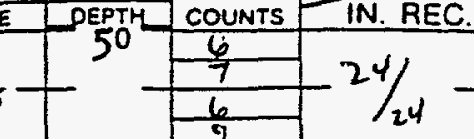

1
1
7
7
7

9

\.

3

3

3

3

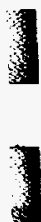

BORING NO. NASA-11 DATE $8 / 19 / 96$ DESCRIPTION

REC. OLOGY

CLAY, SILTY, SLILATLY SANDY, BLUISN GESYY STIFF

WITN TNW BEDS of SNELLS or CCAYEY SAND.

Total Deith e $52 \mathrm{fr}$ 


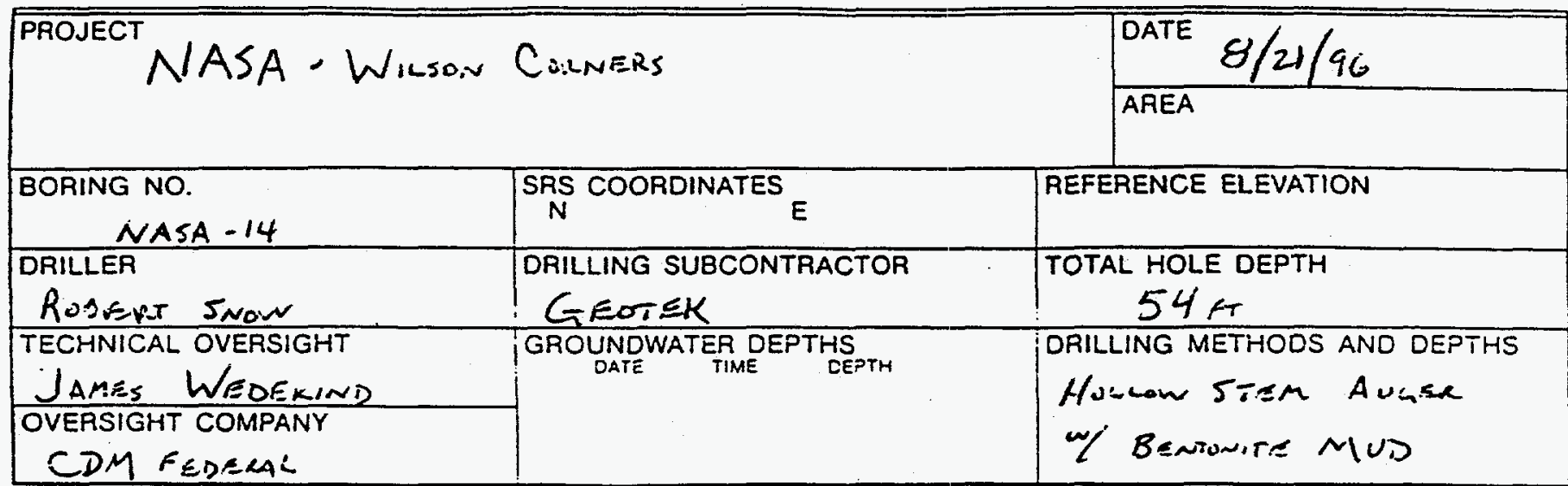

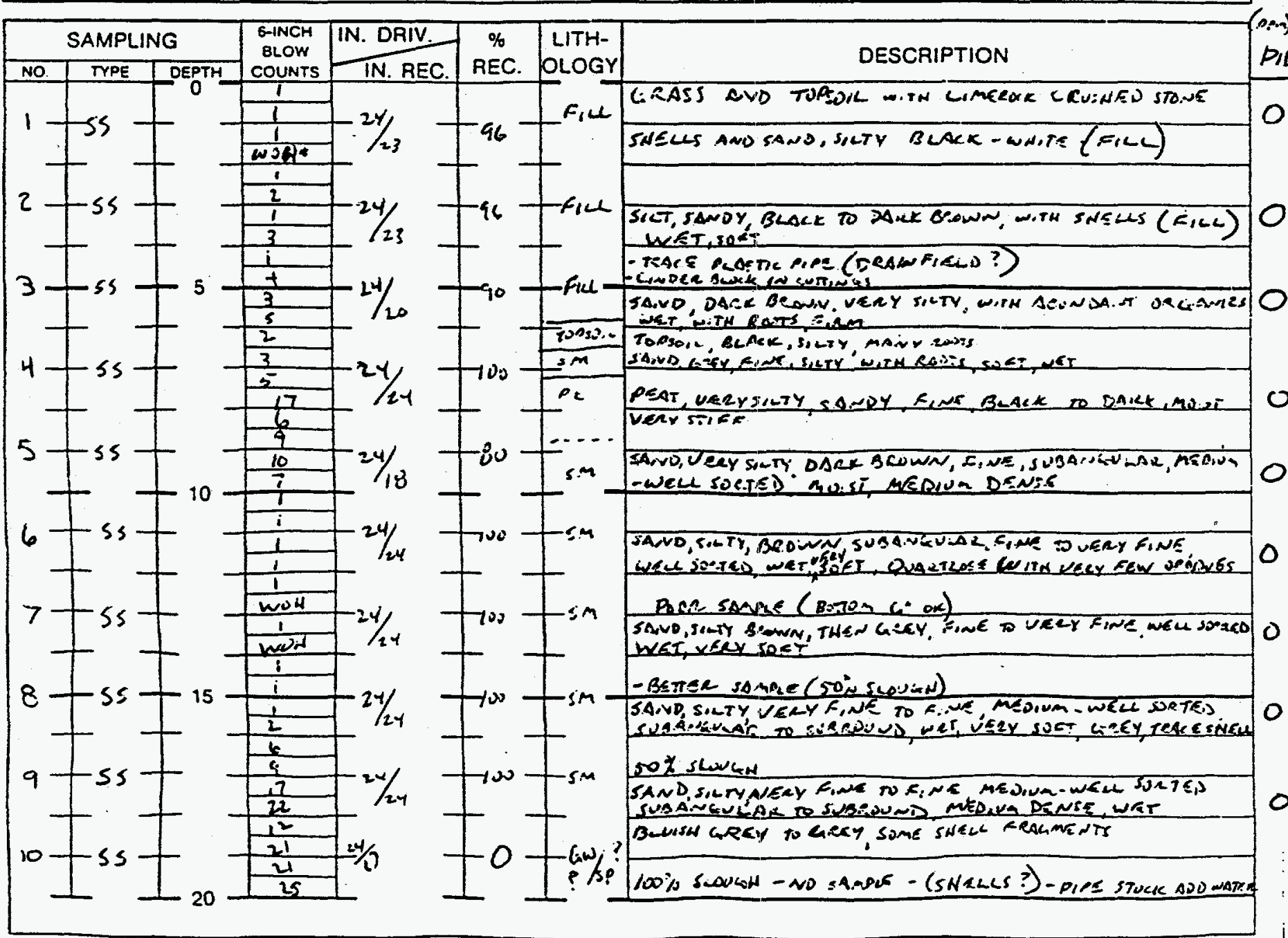

$$
\text { * won- weight on hamea }
$$

Samples Submitted for Laboratory Tests
NOTE: Standard Penetration Resistance is Sum of Blows for 2nd $-6^{*}$ and 3rd - 6" to Drive 1-3/8" 1.D., 2" O.D. Split Barrel Sampler with 140 pound hammer falling 30 inches. 
PID abua $(P+m)$ butyon 0

$6-13(9)$ 4.8

3.5

$7 \cdot 12$

1.2

0

0

$20-30$

iv-20

$15-20$

$10.200 \mathrm{sm}$

30-3NSLLS

$3 \cdot \operatorname{sm}$

0

0

0
Samples Submilled for Laboratory Tests

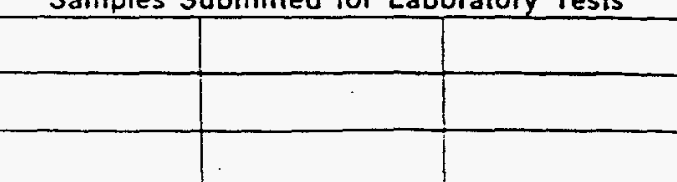

NOTE: Standard Penetration Resistance is Sum of Blows for 2nd $-6^{\prime \prime}$ and 3rd - 6" to Drive 1-3/8" I.D. 2" O.D. Split Barrel Sampler with 140 pound hammer falling 30 inches. ralues $(<0.5$ p. thenely 
STSS

SOIL BORING LOG

PAGE $3 \quad$ OF

\begin{tabular}{|c|c|c|c|c|c|c|}
\hline PROJECT NASA & WILso, & $N \operatorname{Cos} N$ & & & BORING NO. N & DATE $8 / 24 / 96$ \\
\hline SAMPLING & $\begin{array}{l}\text { 6-INCH } \\
\text { BLOW }\end{array}$ & IN. DRIV. & $\%$ & LITH- & DES & \\
\hline
\end{tabular}

$\int$ $26-55+\frac{1}{3}+24 / 23$

$-56+5 M$

SANO, SUTY, CLAYGY BLUE-LESY, ELNE-VERY F,NE

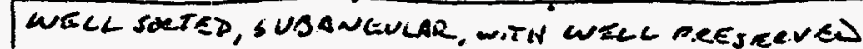

SNECLS, OCCAEIONALGY MAMUNATEO

O

1

?

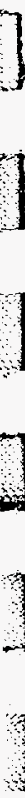

3
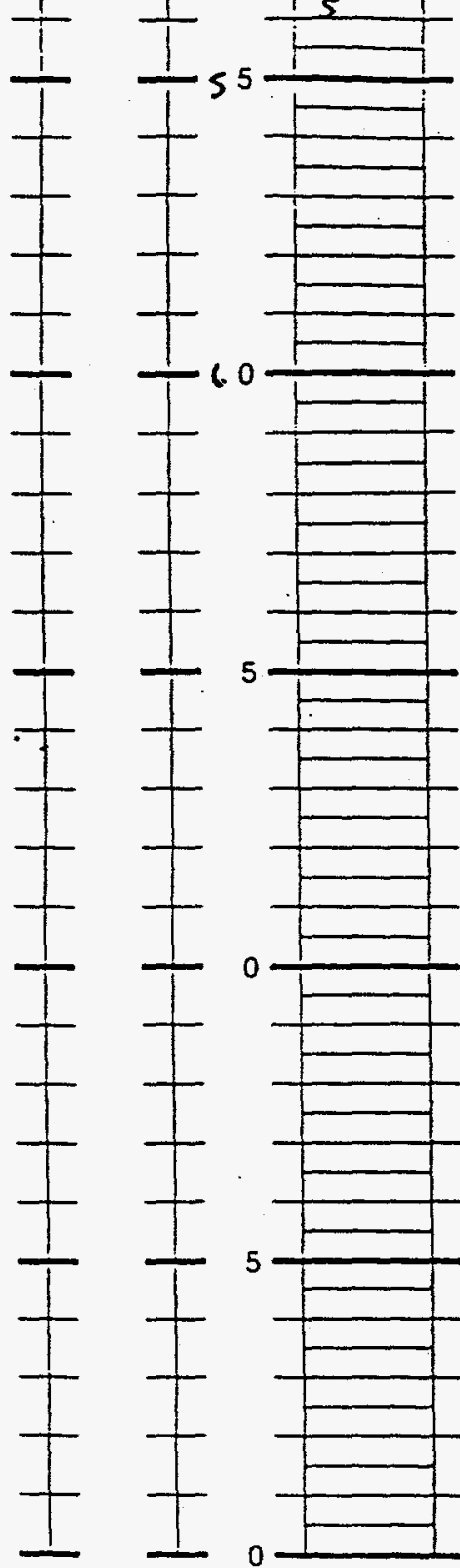

$\frac{a}{5}+$

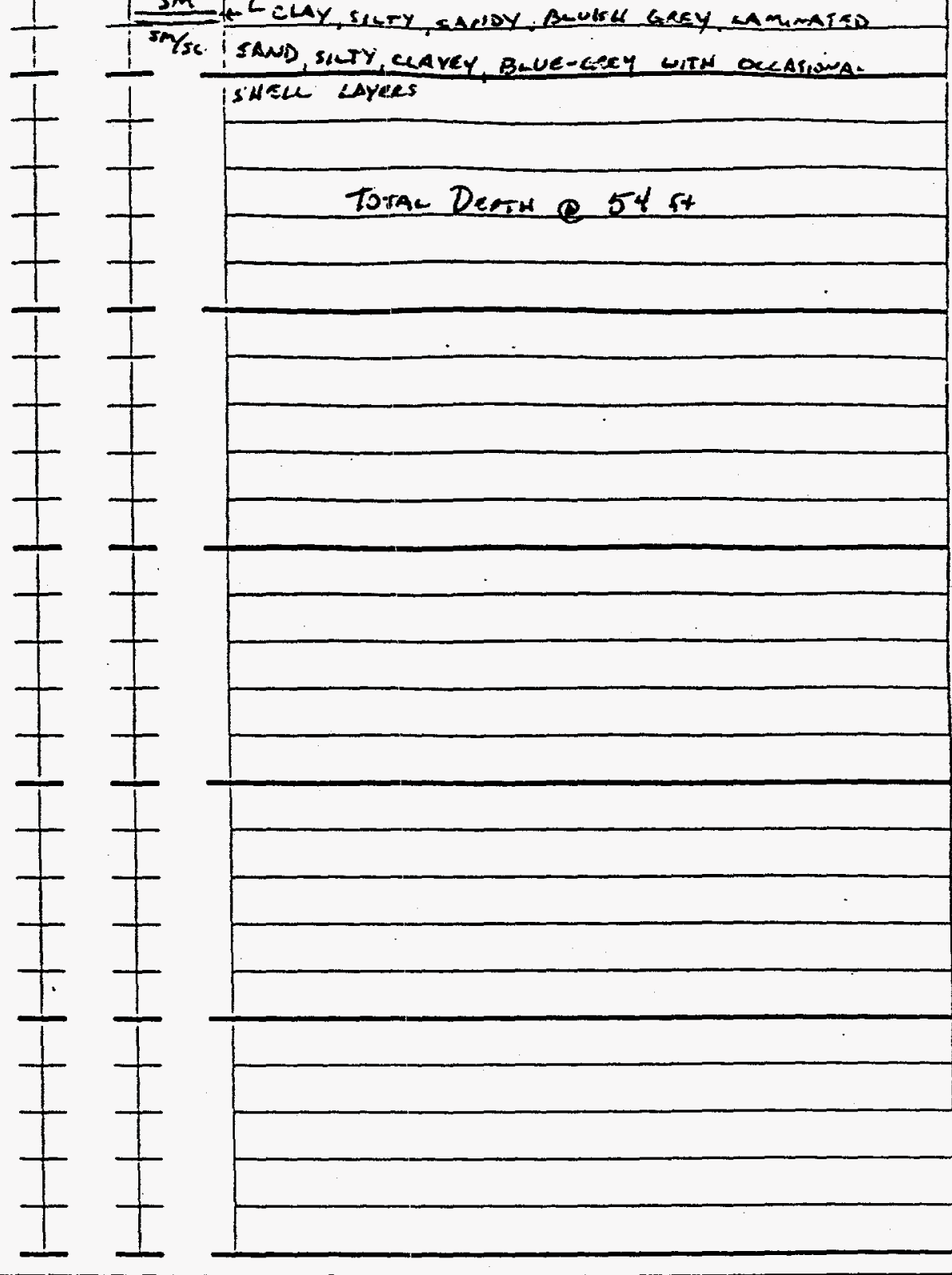

s.

NOTE: Standard Penetration Resistance is Sum of Blows lo 2nd - 6" and 3rd - 6" to Drive 1-3/8" 1.D., 2" O.D. Split Barrel Sampler with 140 pound hammer falling 30 inches. 


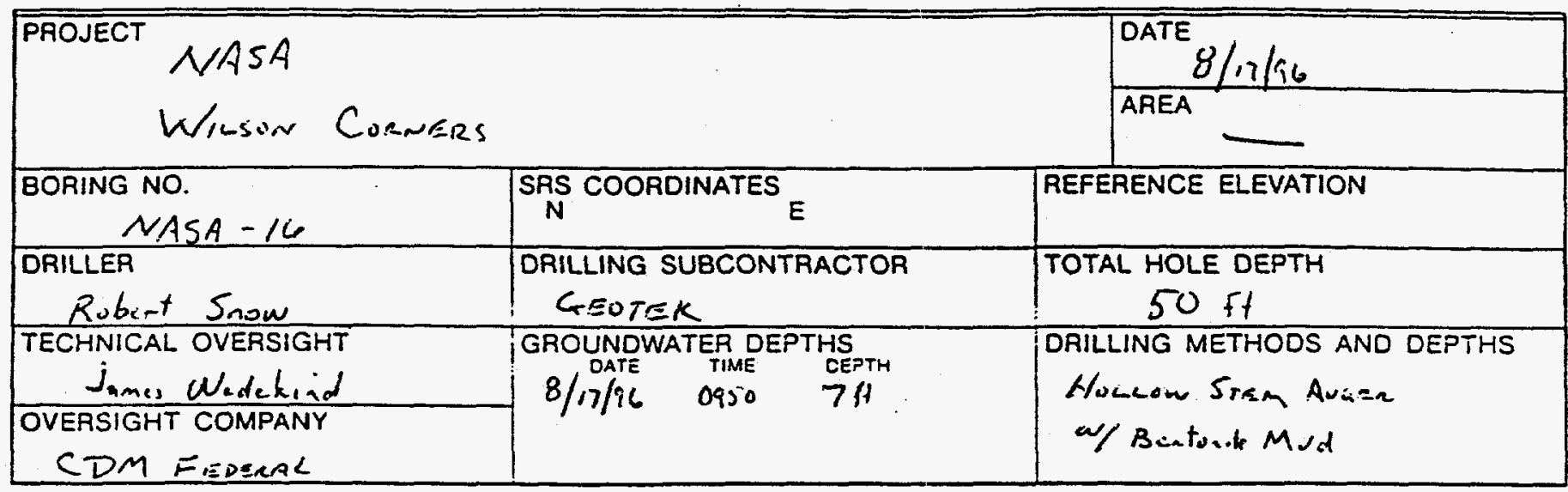

\section{DESCRIPTION}
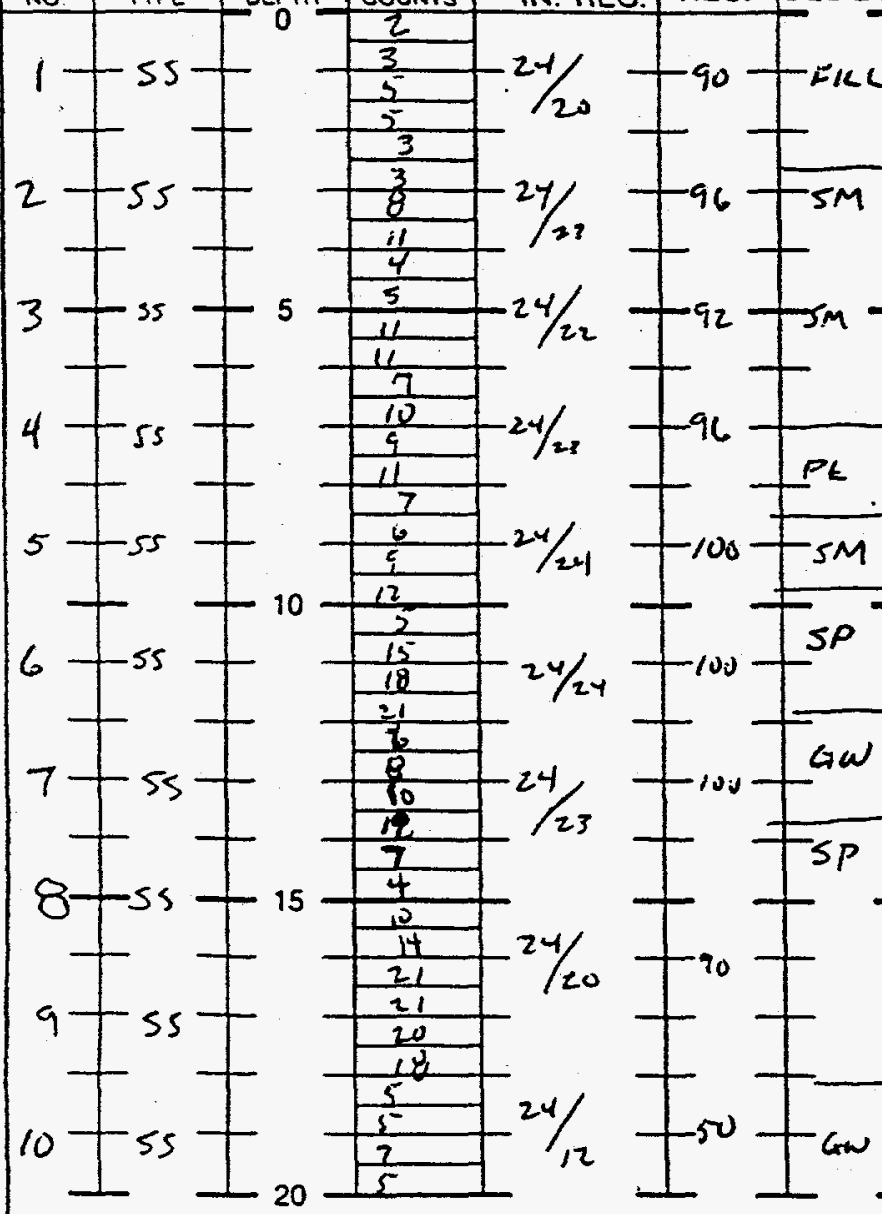

ToRsoin AND LIMECOCK, DARK SEONN SILTY SANDY

LeODES TO PEAT, sANAY, at Base (FILL)

SANO SWIY DAKL RENM FINE SUBONGWIC

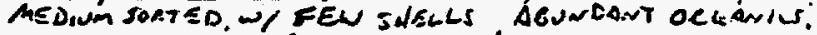

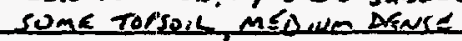


SRS

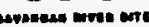

SOIL BORING LOG

PROJECT NASA WILSON COCNAAS

SAMPLING

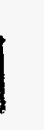

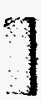

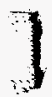

7

1

1

3

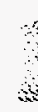

?

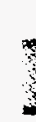

\begin{tabular}{l|c|c|}
$\%$ & LITH- \\
REC. & OLOGY \\
\hline & 100 & $5 P /$ \\
\hline & 100 & \\
\hline & & \\
\hline
\end{tabular}
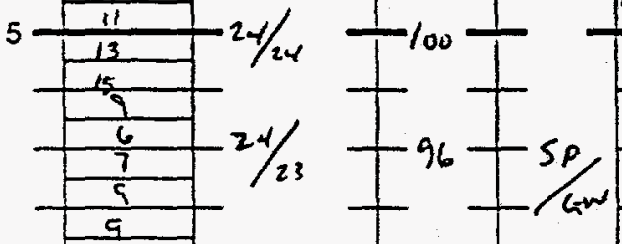

$24 / 24$

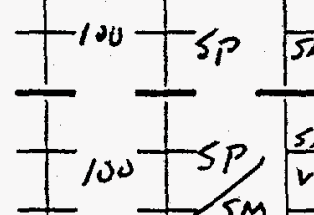
SAnid

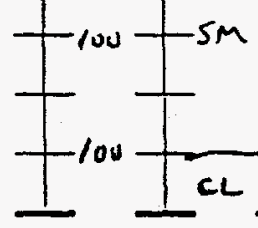

BORING NO.NASA-16 DATE $8 / 17 / 5 C$

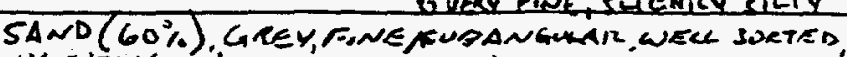

SHELCS $(40 \%)$ VARIBBCY CODEED, GEEY, WHITE, BLACK, TAN

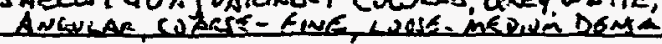

SAND AND SHELS AE AQUVE

15NEUS $(70 \%)$, UARIABLY COWRED. WARE - FINE, AS ABOVE

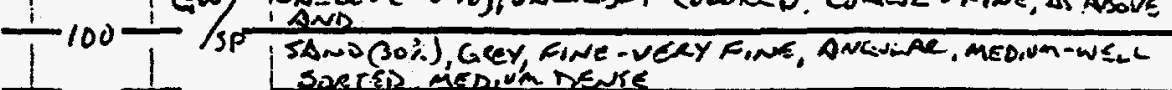

SASELS At AOUVE

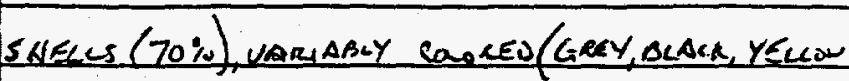

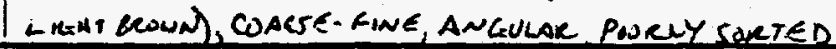

WW/ SAND (30\%), GREY, SLI ENTLY SILTY, SUBANEULAR,

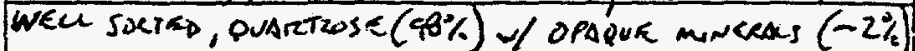

GRADES TO SAND, SAECCY, MEDIUM DKNSE

SAND (70\%), GRE, SULATLY SILTY FINE TO VERY FINE.

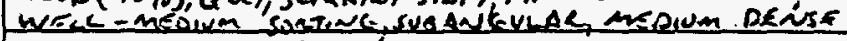

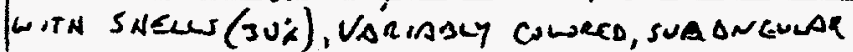

BEOMES WUATEC GREY, TAN C $36 \mathrm{fH}$

SAND (80\%) LCANT CRESY FINE, wECL-MED,UM SORTINE, U/SNELLS ( $20 \%$ ), LRGNT SLOWN, GRCY, CUARE, BRNKEN, ANEVARE

SAND DS ABDVE EXCEPT SWGHTLY BLUISH-GeKSN rugintry jLTY

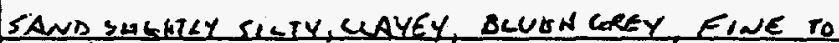

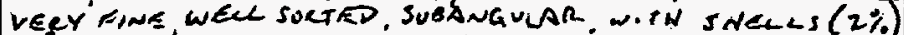
CONCSE- T, INA DETEV WHALR

SAND, SRTY, CCAYEY. BLUISU G CEY, E, INE. TD VEYY FINE

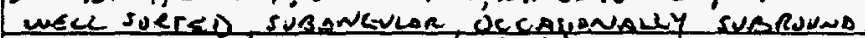

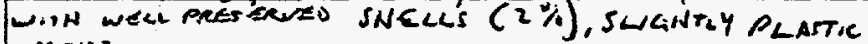

SAND, AI ABUVE, SNGHTLY MORE SAELL FeALmGNTI

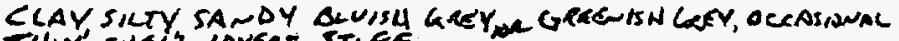

DESCRIPTION

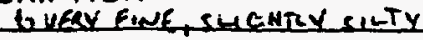

Maist

Ope.

\section{$P I D$ \\ Iren \\ osp \\ Oppa \\ Opm \\ $O_{p / m}$}

Opph

Ophept

Optapet

Opp HAIN SHELL LAver ST, fR
Samples Submitted for Laboratory Tests

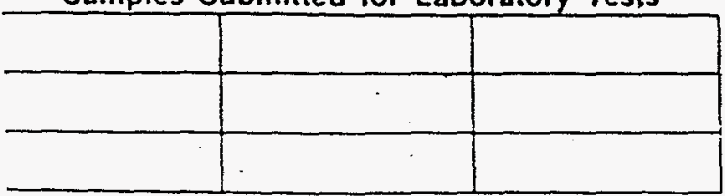

NOTE: Standard Penetration Resistance is Sum of Blows for 2nd - 6" and 3rd - 6" to Drive 1-3/8" 1.D.. 2" O.D. Split Barrel Sampler with 140 pound hammer falling 30 inches. 
PROJECT

NASA - Wilson Cerners

BORING NO.

\section{DRILLER}

NASA - 17

Rubert Snes

TECHNICAL OVERSIGHT

James Wodekind

1

OVERSIGHT COMPANY

(a) Federal
${ }_{N}^{S R S ~ C O O R D I N A T E S ~}$

DRILLING SUBCONTRACTOR

Geotek

GROUNDWATER DEPTHS
DATE $8 / 29 / 96$

REFERENCE ELEVATION

TOTAL HOLE DEPTH

$50 \mathrm{ft}$

DRILLING METHODS AND DEPTHS

Honcow STEM Avsees

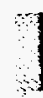

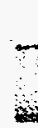

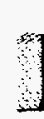

1

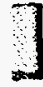

1

3

I

\begin{tabular}{|c|c|c|c|c|c|c|}
\hline \multicolumn{3}{|c|}{ SAMPLING } & \multirow{2}{*}{$\begin{array}{c}\text { 6-INCH } \\
\text { BLOW } \\
\text { COUNTS }\end{array}$} & \multirow{2}{*}{\begin{tabular}{|c|} 
IN. DRIV. \\
IN. REC.
\end{tabular}} & \multirow{2}{*}{$\begin{array}{c}\% \\
\text { REC. }\end{array}$} & \multirow{2}{*}{$\begin{array}{l}\text { LITH- } \\
\text { OLOGY }\end{array}$} \\
\hline NO & TYPE & & & & & \\
\hline
\end{tabular}

DESCRIPTION
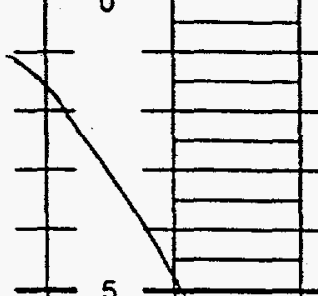

$+1$

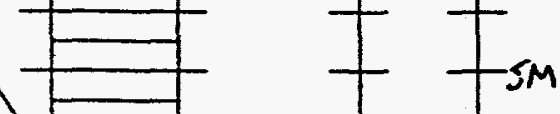

5

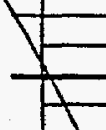

$-$
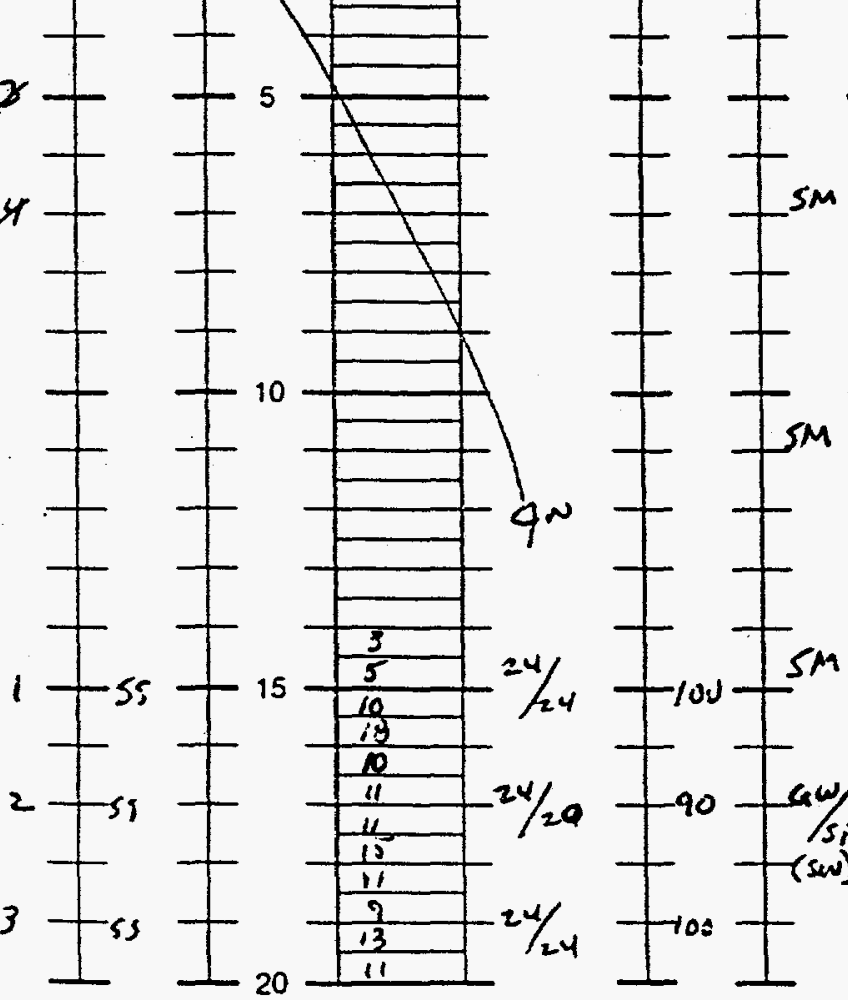

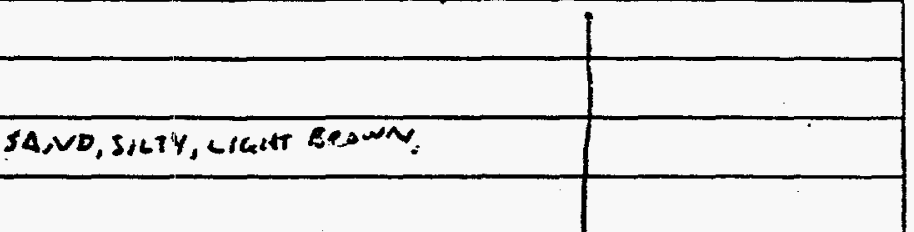

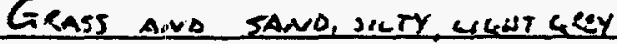

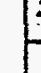

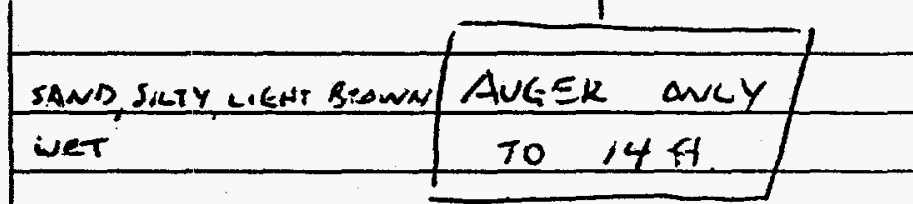

\begin{tabular}{|l|l|}
\hline & \\
\hline & \\
\hline & \\
\hline & \\
\hline & \\
\hline & \\
\hline & \\
\hline & \\
\hline & \\
\hline & \\
\hline & \\
\hline
\end{tabular}

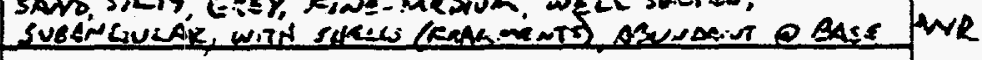

$+100+5$

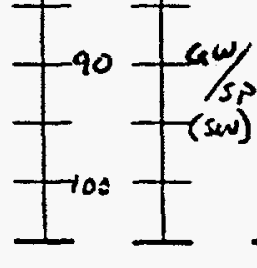

SAND (50\%), ILIENTLY SRLTY, GECY, FINE, WEUL SNAED, SUSANGULAR with

SWECLS $(50-20 \%)$, MOSR Y \&R CY, MEUINM SUCTED

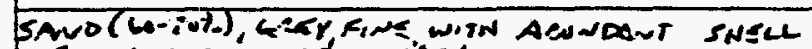

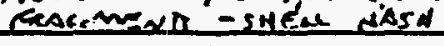

Samples Submitted for Laboratory Tests

\begin{tabular}{|l|l|l|}
\hline & & \\
\hline & & \\
\hline & & \\
\hline
\end{tabular}

\section{* PID Malfundin. - humatit?}

NOTE: Standard Penetration Resistance is Sum of Blows for 2nd - 6" and 3rd - 6" 10 Drive 1-3/8" 1.D. 2" O.D. Split Barrel Sampler with 140 pound hammer falling 30 inches 


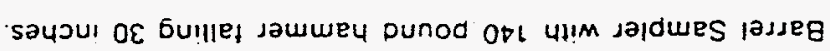

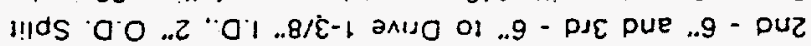
sol smolg to wns s! asueıs!say uollestauad prepuels : $310 \mathrm{~N}$

Hims wery hass apeask

)

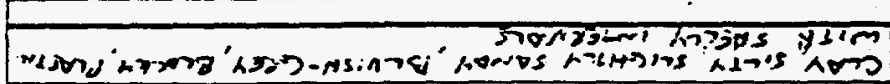

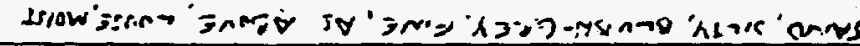

O

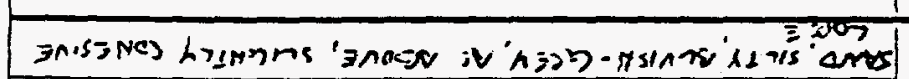

○

C

10

\section{$\bigcirc$}

$\rightarrow$ -Sanivaco

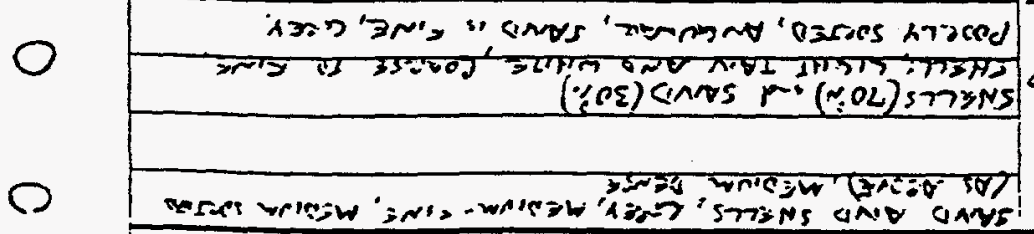

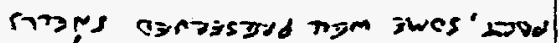

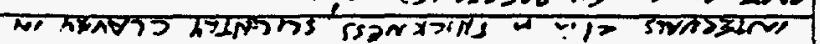

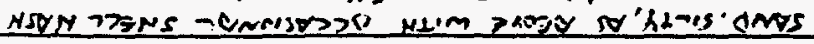

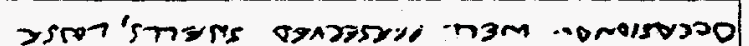

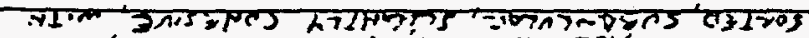

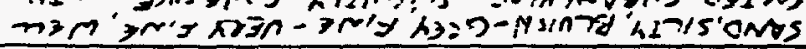

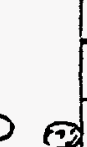

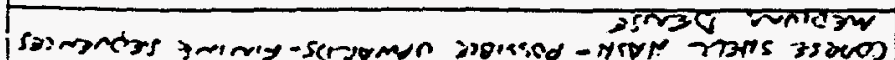

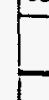

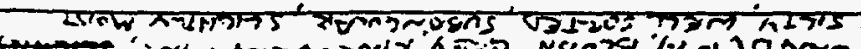

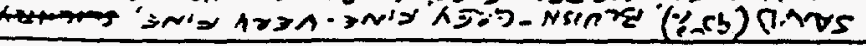

0

an 1

and

S'd

zinsos nnesw

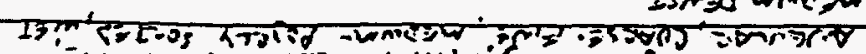

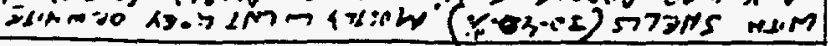

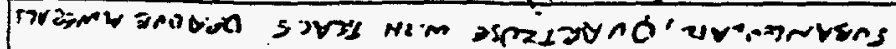

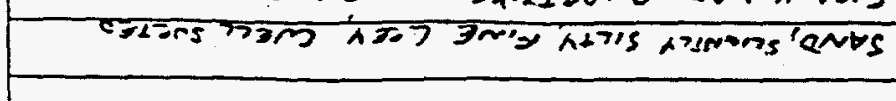

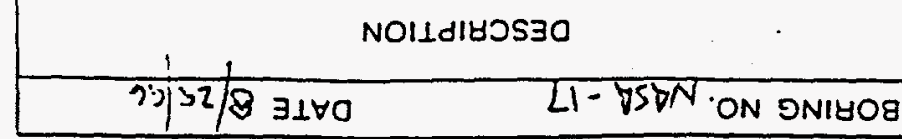

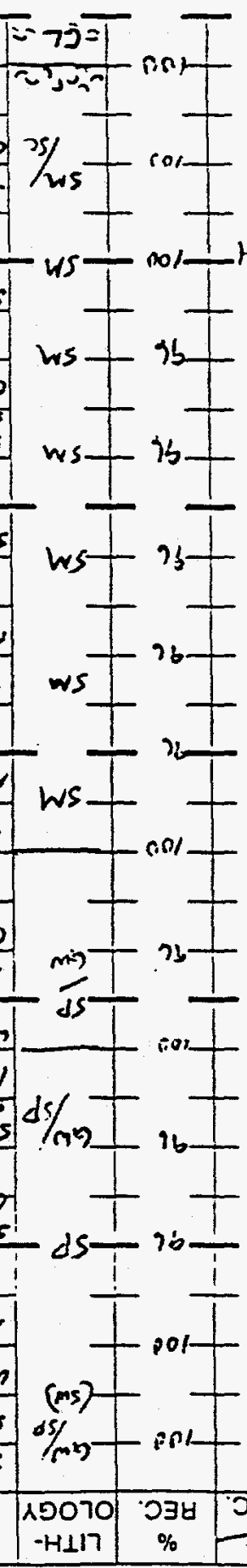

$r^{2}$

in F

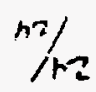
$r^{2} / r^{2}$ $22 /$ $[2 / 1+2$

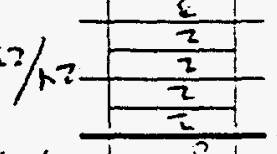

(2) he

$22 / \mathrm{r}=$ $5 / 2+$ $h=$ sim $m / r_{2}$ $\varepsilon=1$ $/ r=$ $E=1 / T_{2}$ \begin{tabular}{|c|}
$\frac{9}{2}$ \\
\hline की \\
\hline
\end{tabular} $n^{2}$ $2 / n=$ $\frac{s}{2}$

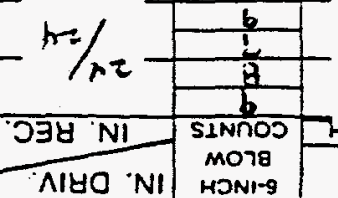

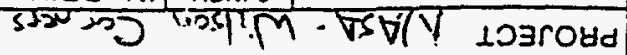


sauju! of 6u!!lej sawwey punod opl 4l!m sajduses lasseg

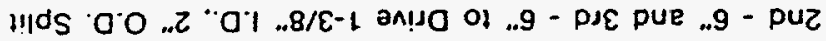
sol smola to uns s! asuelsisay uolnejlauad pjepueis : $310 \mathrm{~N}$

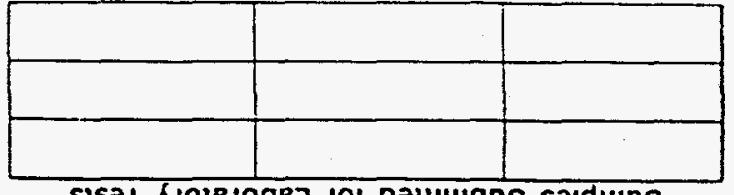

s\}sal kojesoqe7 sol pal!uquS sajdwes

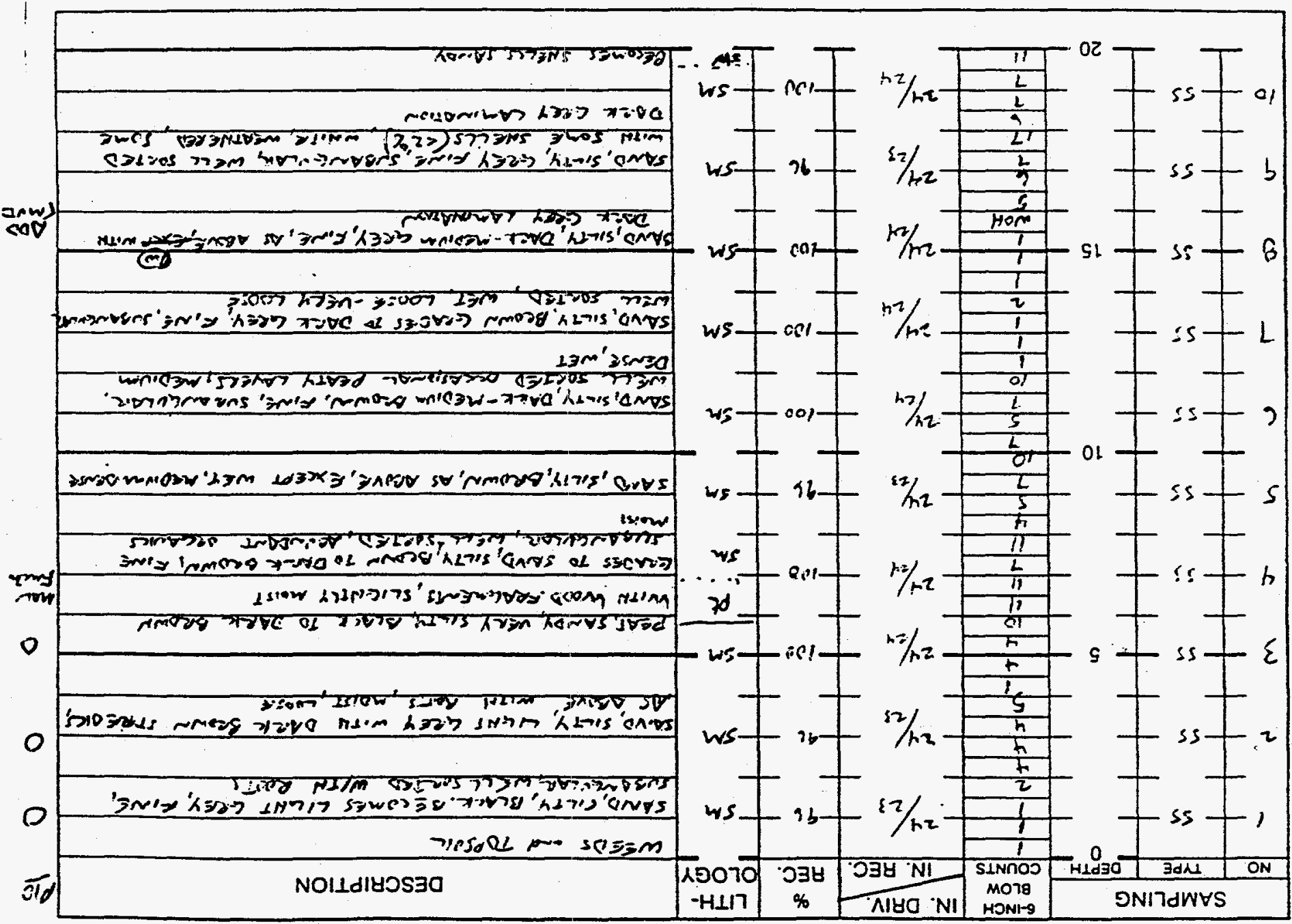

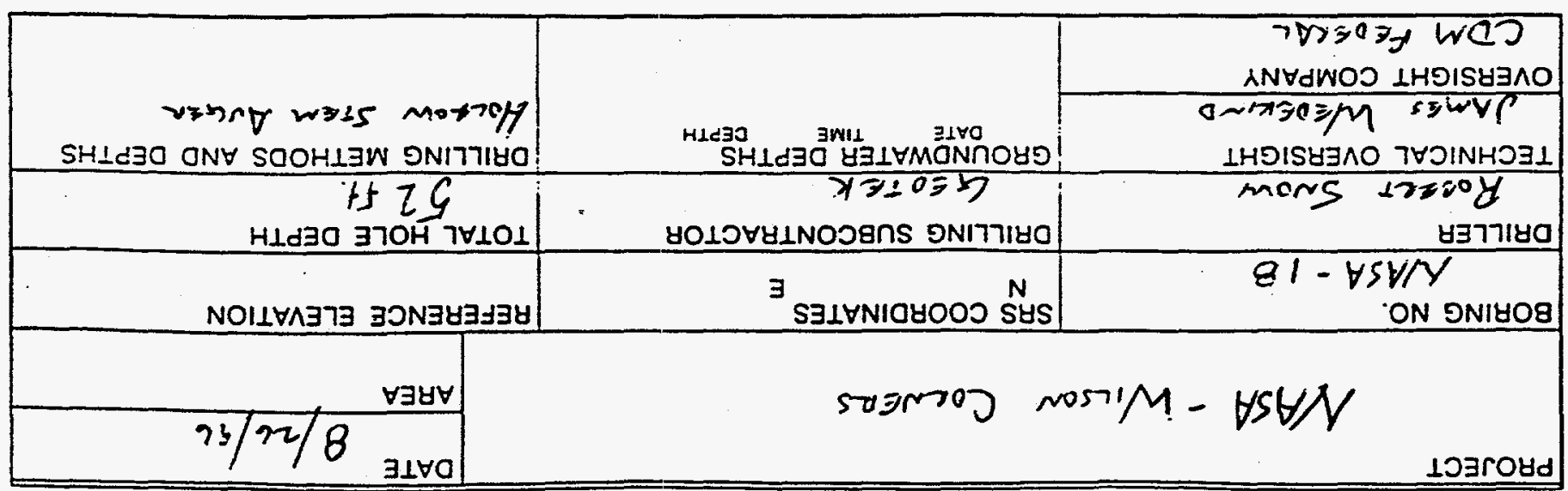

$\varepsilon=0 \quad I$ INWd

פט פ
SIS 
sayou! of 6u!lle! sawuey punod otl 4!!M sajdures lasseg

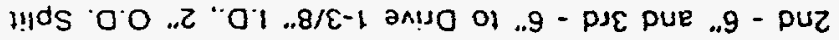

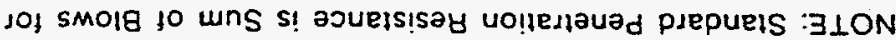

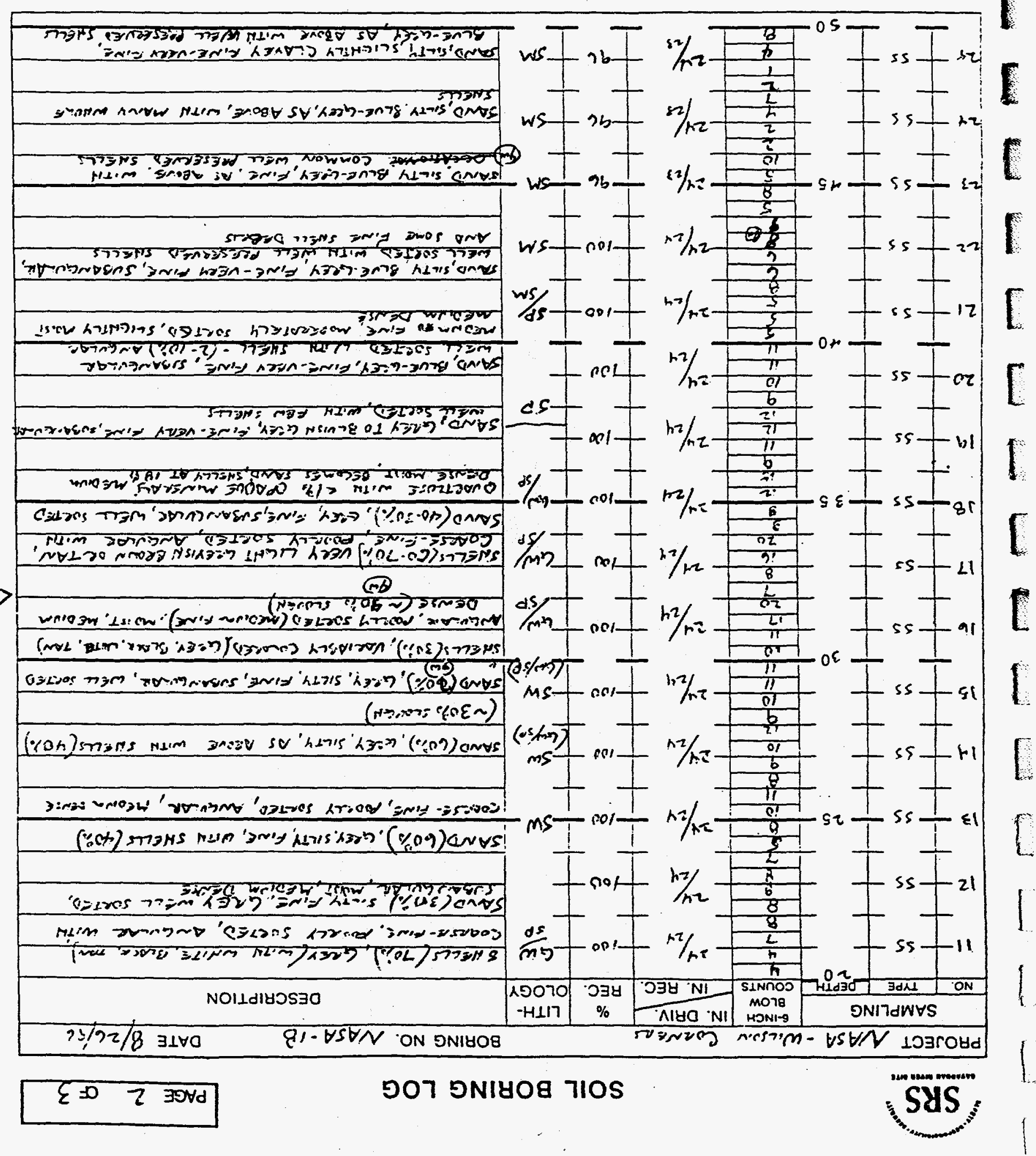




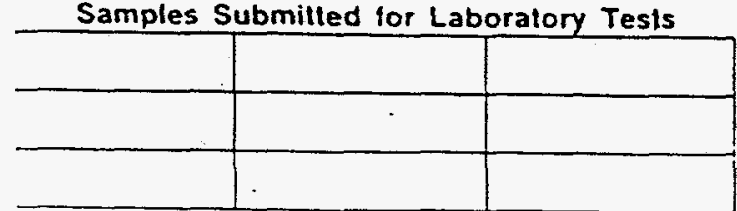

SHELLS wEL PEISECSED

on LAYßRS - $1 / 4+x .46$ se

DISEMUNATED in MATR.X

$$
\text { Tota DETNe } 52 \mathrm{fl} \text {. }
$$

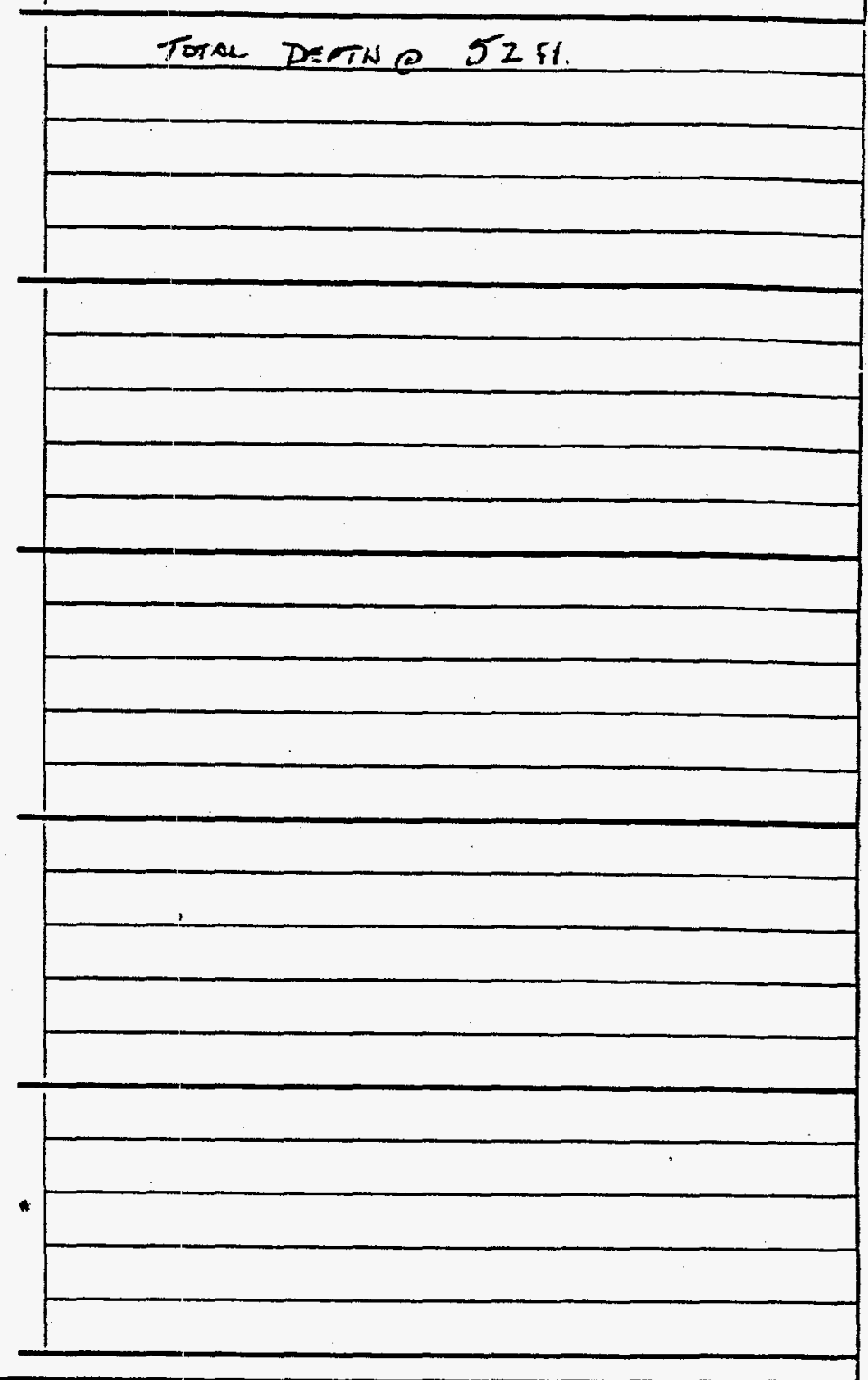

NOTE: Standard Penetration Resistance is Sum of Blows for 2nd - 6" and 3rd - 6" to Drive 1-3/8" 1.D., 2" O.D. Split Barrel Sarnpler with 140 pound hammer falting 30 inches. 


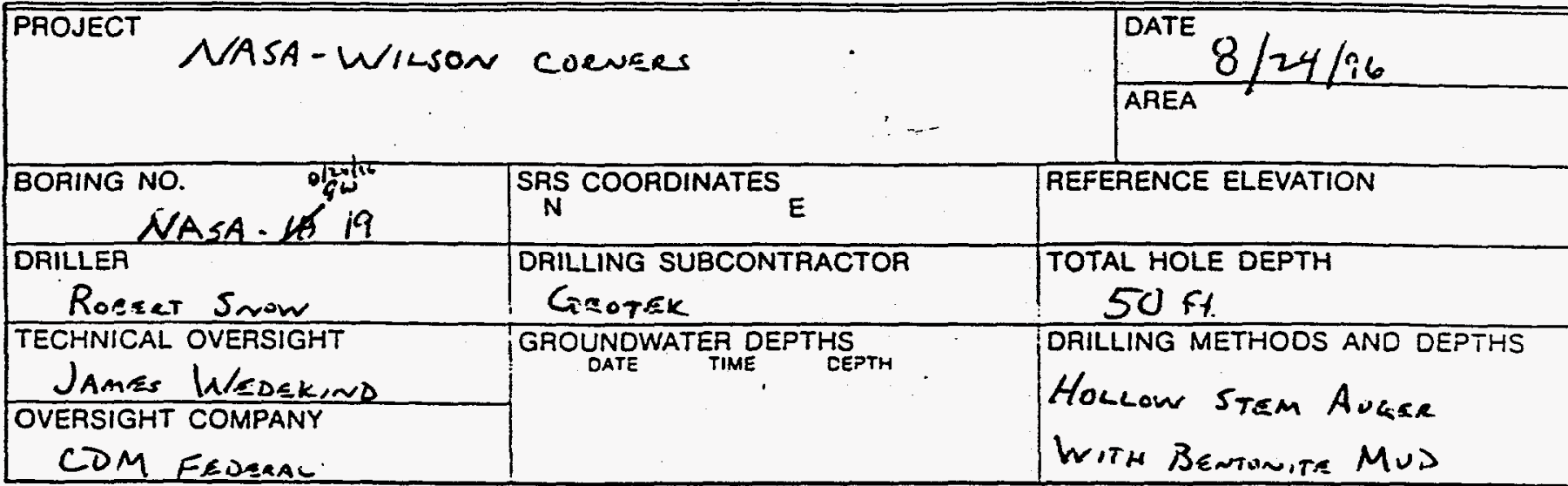
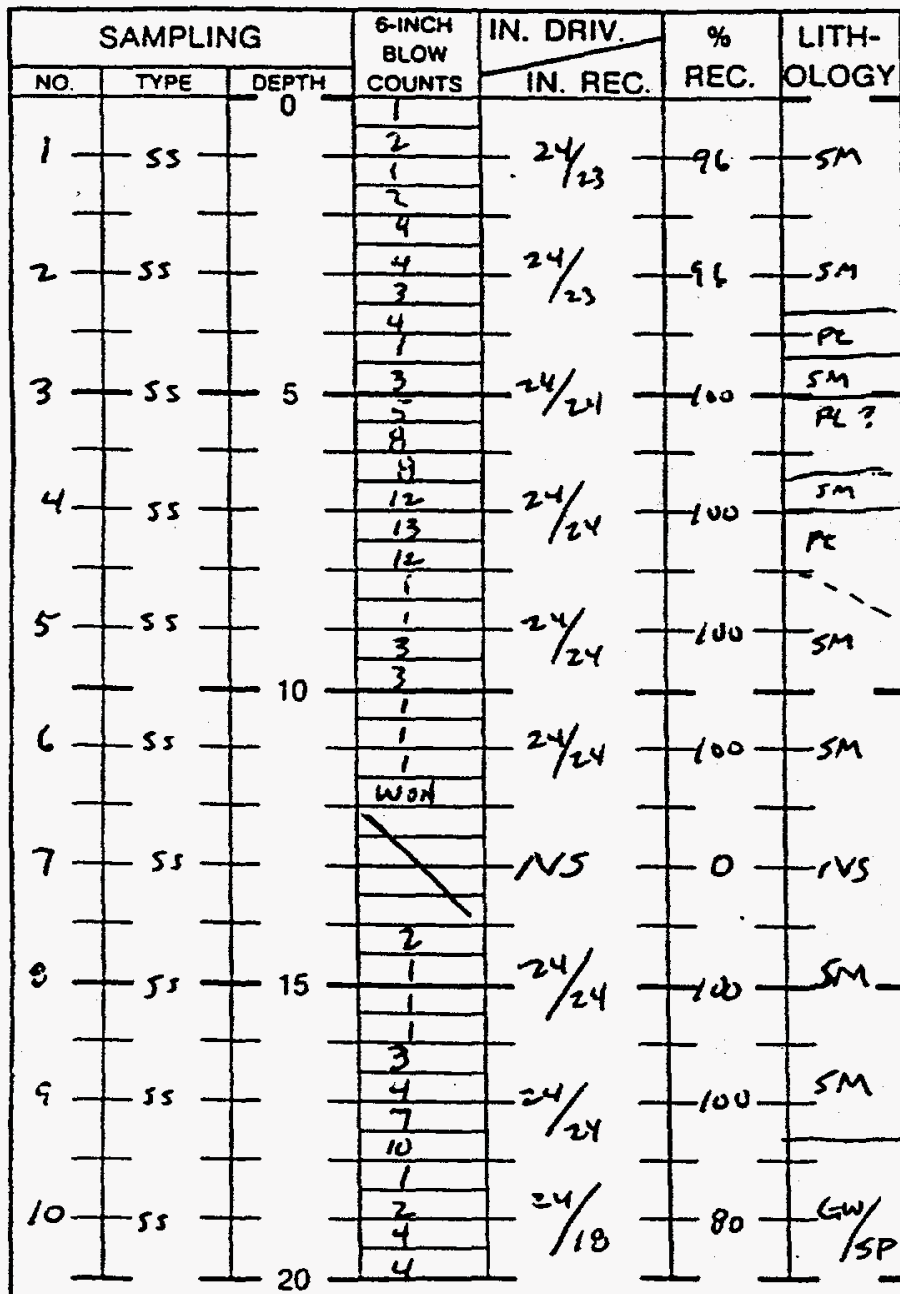

DESCRIPTION

GRASS

SAND, SETY, EIGNT LREY WITN BLAK STREAKS

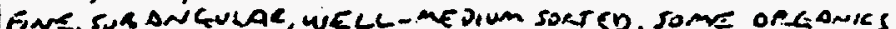
Coof's, Dey, Loere

SAVIS, SLLTY LIGWT CNEY ndAT

DEAT VEeY SILTY, SANOY, ABUNDONT DEGANILS, RuJis CRY

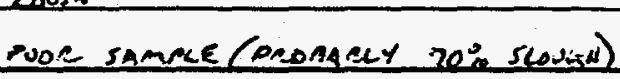

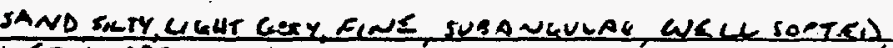
WT, EPACES CECY-ALALR

Ear, as above, meivm oenca

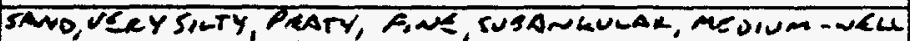

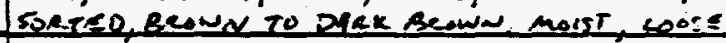

SANO, DEEY SMTY, DARK BNOWN - BEOND, SATUEOTEA os sevike

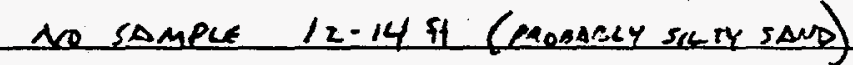

SAND, SIRY BCoWW/SH - GEAY FINE, SUBANGULAR,

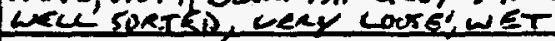

SAIVS, S/LTY, GREY AND MO TLED O.ROWN, FINE, SUSANGULAR, WELL SARTED MED.UM DCNSE, WET

SHELCS (6O-70\%) VACIASLY COLORED (C.TCY, w WITC)

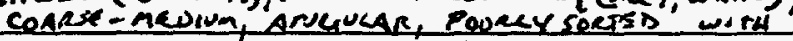

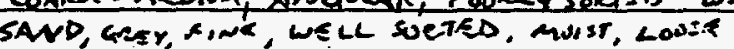

Samples Submitted for Laboratory Tests

\begin{tabular}{|l|l|l|}
\hline & & \\
\hline & & \\
\hline & & \\
\hline
\end{tabular}

NOTE: Standard Penetration Resistance is Sum of Blows for 2nd - 6" and 3rd - 6" to Drive 1-3/8" 1.0.. 2" O.D. Split Barrel Sampler with 140 pound hammer falling 30 inches. 


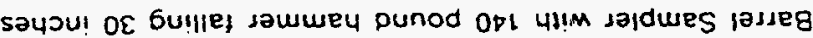

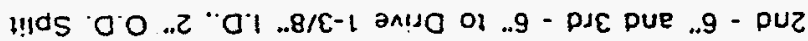

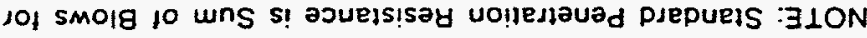

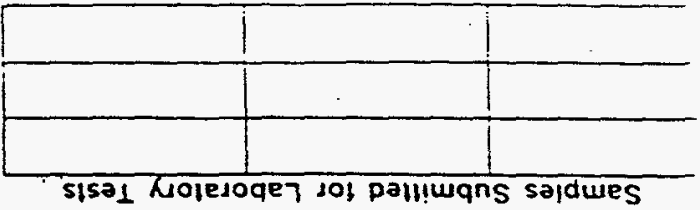

$.0-50.4$

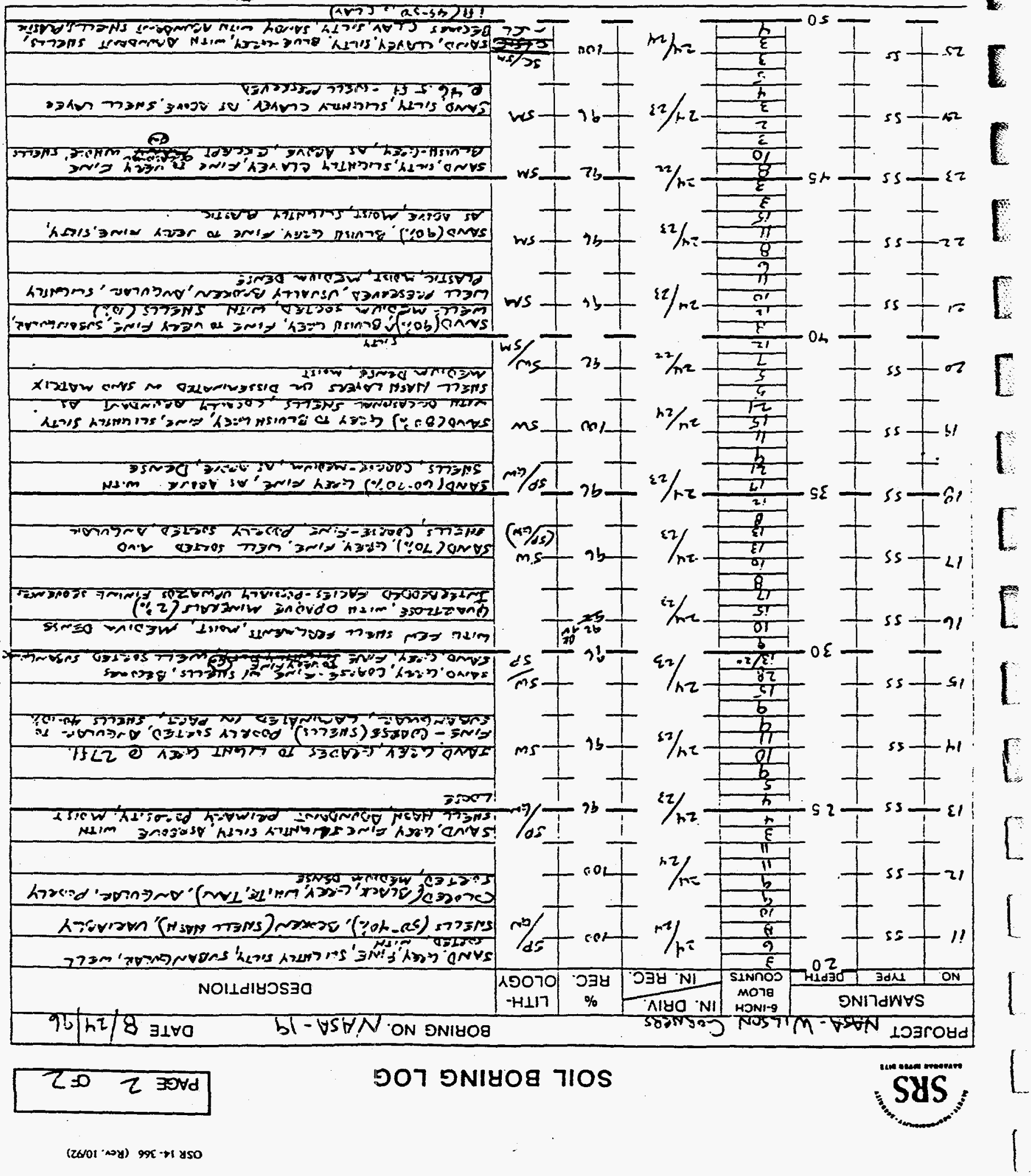

$(\ln 2)$ Crw ady $>$ $(13)$ Hingens anw sory

enw ser

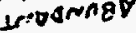


Appendix B

\author{
Results from Headspace Analysis \\ of Sediment Samples Collected \\ at the Wilson's Corner Site
}




\begin{tabular}{|c|c|c|c|c|c|c|c|}
\hline \multirow[b]{2}{*}{ Loc. Id } & \multicolumn{2}{|c|}{ feet } & \multicolumn{4}{|c|}{$\mathrm{ug} / \mathrm{kg}$} & \multirow[b]{2}{*}{ Samp. Id } \\
\hline & depth & elevation & $\mathrm{VCl}$ & $F-113$ & cis-DCE & TCE & \\
\hline NASA-1 & 1 & 6.25 & $<0.001$ & $<0.001$ & $<0.001$ & $<0.001$ & NASA0100 \\
\hline NASA-1 & 3 & 4.25 & $<0.001$ & $<0.001$ & $<0.001$ & $<0.001$ & NASA0101 \\
\hline NASA-1 & 5 & 2.25 & $<0.001$ & $<0.001$ & $<0.001$ & $<0.001$ & NASA0102 \\
\hline NASA-1 & 7 & 0.25 & $<0.001$ & $<0.001$ & 0.005 & $<0.001$ & NASA0103 \\
\hline NASA-1 & 9 & -1.75 & $<0.001$ & $<0.001$ & 0.003 & $<0.001$ & NASA0104 \\
\hline NASA-1 & 11 & -3.75 & $<0.001$ & $<0.001$ & $<0.001$ & $<0.001$ & NASA0105 \\
\hline NASA-1 & 13 & -5.75 & $<0.001$ & $<0.001$ & $<0.001$ & $<0.001$ & NASA0106 \\
\hline NASA-1 & 15 & -7.75 & $<0.001$ & $<0.001$ & $<0.001$ & $<0.001$ & NASA0107 \\
\hline NASA-1 & 17 & -9.75 & $<0.001$ & $<0.001$ & $<0.001$ & $<0.001$ & NASA0108 \\
\hline NASA-1 & 19 & -11.75 & $<0.001$ & $<0.001$ & $<0.001$ & $<0.001$ & NASA0109 \\
\hline NASA-1 & 21 & -13.75 & $<0.001$ & $<0.001$ & $<0.001$ & $<0.001$ & NASA0110 \\
\hline NASA-1 & 23 & -15.75 & $<0.001$ & $<0.001$ & $<0.001$ & $<0.001$ & NASA0111 \\
\hline NASA-1 & 25 & -17.75 & $<0.001$ & $<0.001$ & 0.005 & $<0.001$ & NASA0112 \\
\hline NASA-1 & 27 & -19.75 & $<0.001$ & $<0.001$ & $<0.001$ & $<0.001$ & NASA0113 \\
\hline NASA-1 & 29 & -21.75 & $<0.001$ & $<0.001$ & $<0.001$ & $<0.001$ & NASA0114 \\
\hline NASA-1 & 31 & -23.75 & $<0.001$ & $<0.001$ & $<0.001$ & 0.002 & NASA0115 \\
\hline NASA-1 & 33 & -25.75 & $<0.001$ & $<0.001$ & 0.002 & 0.004 & NASA0116 \\
\hline NASA-1 & 35 & -27.75 & $<0.001$ & $<0.001$ & 0.005 & 0.003 & NASA0117 \\
\hline NASA-1 & 37 & -29.75 & $<0.001$ & $<0.001$ & 0.007 & 0.020 & NASA0118 \\
\hline NASA-1 & 39 & -31.75 & $<0.001$ & $<0.001$ & 0.019 & 0.042 & NASA0119 \\
\hline NASA-1 & 41 & -33.75 & 0.294 & 0.002 & 4.756 & 2.739 & NASA0120 \\
\hline NASA-1 & 43 & -35.75 & 1.016 & 0.008 & 18.661 & 9.805 & NASA0121 \\
\hline NASA-1 & 45 & -37.75 & 0.256 & 0.001 & 3.873 & 2.022 & NASA0122 \\
\hline NASA-1 & 47 & -39.75 & 0.883 & 0.003 & 7.349 & 2.174 & NASA0123 \\
\hline NASA-1 & 49 & -41.75 & 0.727 & 0.002 & 3.425 & 1.011 & NASA0124 \\
\hline NASA-1 & 51 & -43.75 & 0.913 & 0.002 & 2.986 & 0.481 & NASA0125 \\
\hline NASA-1 & 52.5 & -45.25 & $<0.001$ & $<0.001$ & $<0.001$ & $<0.001$ & NASA0126 \\
\hline NASA-1 & 53.5 & -46.25 & 0.206 & $<0.001$ & $<0.001$ & $<0.001$ & NASA0127 \\
\hline NASA-2 & 1 & 7.70 & 0.896 & $<0.001$ & $<0.001$ & $<0.001$ & NASA0200 \\
\hline NASA-2 & 3 & 5.70 & $<0.001$ & $<0.001$ & $<0.001$ & $<0.001$ & NASA0201 \\
\hline NASA-2 & 4.5 & 4.20 & 0.766 & $<0.001$ & $<0.001$ & $<0.001$ & NASA0202 \\
\hline NASA-2 & 7.5 & 1.20 & 1.470 & $<0.001$ & 0.075 & $<0.001$ & NASA0204 \\
\hline NASA-2 & 12.5 & -3.80 & $<0.001$ & $<0.001$ & 0.013 & $<0.001$ & NASA0206 \\
\hline NASA-2 & 14 & -5.30 & 0.242 & $<0.001$ & 0.009 & $<0.001$ & NASA0207 \\
\hline NASA-2 & 22.5 & -13.80 & 1.053 & $<0.001$ & $<0.001$ & 0.002 & NASA0212 \\
\hline NASA-2 & 29.5 & -20.80 & 0.398 & $<0.001$ & $<0.001$ & $<0.001$ & NASA0215 \\
\hline NASA-2 & 35 & -26.30 & $<0.001$ & $<0.001$ & $<0.001$ & $<0.001$ & NASA0218 \\
\hline NASA-2 & 39.5 & -30.80 & $<0.001$ & $<0.001$ & 0.033 & 0.009 & NASA0220 \\
\hline NASA-2 & 41 & -32.30 & 0.225 & $<0.001$ & $<0.001$ & $<0.001$ & NASA0221 \\
\hline NASA-2 & 43 & -34.30 & $<0.001$ & $<0.001$ & 0.036 & 0.002 & NASA0222 \\
\hline NASA-2 & 45 & -36.30 & $<0.001$ & $<0.001$ & $<0.001$ & 0.001 & NASA0223 \\
\hline NASA-2 & 47 & -38.30 & $<0.001$ & $<0.001$ & $<0.001$ & $<0.001$ & NASA0224 \\
\hline NASA-2 & 49 & -40.30 & $<0.001$ & $<0.001$ & $<0.001$ & $<0.001$ & NASA0225 \\
\hline NASA-2 & 51 & -42.30 & 0.013 & $<0.001$ & $<0.001$ & $<0.001$ & NASA0226 \\
\hline NASA-2 & 52 & -43.30 & $<0.001$ & 0.001 & $<0.001$ & $<0.001$ & NASA0227 \\
\hline NASA-2 & 53.5 & -44.80 & 0.526 & $<0.001$ & $<0.001$ & $<0.001$ & NASA0229 \\
\hline NASA-3 & 1 & 7.14 & $<0.001$ & $<0.001$ & $<0.001$ & $<0.001$ & NASA0300 \\
\hline
\end{tabular}




\begin{tabular}{|c|c|c|c|c|c|c|c|}
\hline \multirow[b]{2}{*}{ Loc. Id } & \multicolumn{2}{|c|}{ feet } & \multicolumn{4}{|c|}{$\mathrm{ug} / \mathrm{kg}$} & \multirow[b]{2}{*}{ Samp. Id } \\
\hline & depth & elevation & $\mathrm{VCl}$ & $\mathrm{F}-113$ & cis-DCE & TCE & \\
\hline NASA-3 & 3 & 5.14 & 0.781 & $<0.001$ & 0.006 & $<0.001$ & NASA0301 \\
\hline NASA-3 & 5 & 3.14 & 0.784 & 0.012 & 0.130 & 0.054 & NASA0302 \\
\hline NASA-3 & 7 & 1.14 & 0.739 & 1.016 & 3.210 & 1.110 & NASA0303 \\
\hline NASA-3 & 9 & -0.86 & 3.194 & 13.742 & 10.586 & 19.871 & NASA0304 \\
\hline NASA-3 & 11 & -2.86 & 2.954 & 13.390 & 10.191 & 15.005 & NASA0305 \\
\hline NASA-3 & 13 & -4.86 & 0.915 & 5.598 & 7.797 & 5.579 & NASA0306 \\
\hline NASA-3 & 15 & -6.86 & 2.820 & 3.718 & 13.812 & 0.808 & NASA0307 \\
\hline NASA-3 & 17 & -8.86 & 0.906 & 2.972 & 6.844 & 2.137 & NASA0308 \\
\hline NASA-3 & 19 & -10.86 & 0.866 & 3.306 & 5.779 & 1.253 & NASA0309 \\
\hline NASA-3 & 21 & -12.86 & 1.082 & 2.286 & 6.484 & 1.038 & NASA0310 \\
\hline NASA-3 & 23 & -14.86 & 1.027 & 2.906 & 6.886 & 0.810 & NASA0311 \\
\hline NASA-3 & 25 & -16.86 & 0.679 & 2.297 & 5.475 & 0.808 & NASA0312 \\
\hline NASA-3 & 27 & -18.86 & 0.714 & 4.648 & 6.016 & 6.557 & NASA0313 \\
\hline NASA-3 & 29 & -20.86 & 0.288 & 2.988 & 2.160 & 7.478 & NASA0314 \\
\hline NASA-3 & 31 & -22.86 & 0.555 & 2.622 & 4.235 & 4.793 & NASA0315 \\
\hline NASA-3 & 33 & -24.86 & 0.336 & 1.476 & 2.882 & 3.695 & NASA0316 \\
\hline NASA-3 & 35 & -26.86 & 0.436 & 2.074 & 3.253 & 3.745 & NASA0317 \\
\hline NASA-3 & 37 & -28.86 & 0.180 & 16.233 & 0.581 & 3.475 & NASA0318 \\
\hline NASA-3 & 39 & -30.86 & $<0.001$ & 10.193 & 0.092 & 0.345 & NASA0319 \\
\hline NASA-3 & 41 & -32.86 & $<0.001$ & 5.263 & 0.156 & 0.573 & NASA0320 \\
\hline NASA-3 & 43 & -34.86 & 0.124 & 7.819 & 0.370 & 1.067 & NASA0321 \\
\hline NASA-3 & 45 & -36.86 & $<0.001$ & 11.899 & 0.146 & 0.229 & NASA0322 \\
\hline NASA-3 & 47 & -38.86 & $<0.001$ & 5.311 & 0.084 & 0.126 & NASA0323 \\
\hline NASA-3 & 48.5 & -40.36 & 0.331 & 0.325 & 0.956 & 1.569 & NASA0324 \\
\hline NASA-3 & 49.5 & -41.36 & 0.741 & 0.103 & 0.041 & 0.013 & NASA0325 \\
\hline NASA-4 & 1.5 & 6.66 & 0.651 & 0.022 & $<0.001$ & 0.001 & NASA0400 \\
\hline NASA-4 & 3 & 5.16 & $<0.001$ & $<0.001$ & $<0.001$ & $<0.001$ & NASA0401 \\
\hline NASA-4 & 4 & 4.16 & $<0.001$ & 0.010 & 0.225 & 2.300 & NASA0402 \\
\hline NASA-4 & 5.5 & 2.66 & 0.968 & 0.001 & 0.034 & 0.200 & NASA0403 \\
\hline NASA-4 & 8 & 0.16 & $<0.001$ & $<0.001$ & 0.007 & 0.001 & NASA0404 \\
\hline NASA-4 & 11 & -2.84 & $<0.001$ & $<0.001$ & $<0.001$ & $<0.001$ & NASA0406 \\
\hline NASA -4 & 19 & -10.84 & 0.540 & $<0.001$ & $<0.001$ & 0.001 & NASA0410 \\
\hline NASA-4 & 21 & -12.84 & 0.777 & $<0.001$ & $<0.001$ & $<0.001$ & NASA0411 \\
\hline NASA-4 & 23 & -14.84 & $<0.001$ & $<0.001$ & $<0.001$ & $<0.001$ & NASA0412 \\
\hline NASA-4 & 25 & -16.84 & $<0.001$ & $<0.001$ & $<0.001$ & 0.001 & NASA0413 \\
\hline NASA-4 & 27 & -18.84 & $<0.001$ & 0.023 & $<0.001$ & $<0.001$ & NASA0414 \\
\hline NASA-4 & 29 & -20.84 & $<0.001$ & 0.023 & $<0.001$ & 0.002 & NASA0415 \\
\hline NASA-4 & 33 & -24.84 & $<0.001$ & 0.046 & $<0.001$ & 0.003 & NASA0417 \\
\hline NASA-4 & 35 & -26.84 & $<0.001$ & $<0.001$ & $<0.001$ & $<0.001$ & NASA0418 \\
\hline NASA-4 & 37 & -28.84 & $<0.001$ & $<0.001$ & $<0.001$ & $<0.001$ & NASA0419 \\
\hline NASA-4 & 39 & -30.84 & $<0.001$ & $<0.001$ & $<0.001$ & 0.002 & NASA0420 \\
\hline NASA-4 & 41 & -32.84 & $<0.001$ & $<0.001$ & $<0.001$ & $<0.001$ & NASA0421 \\
\hline NASA-4 & 43 & -34.84 & $<0.001$ & $<0.001$ & $<0.001$ & $<0.001$ & NASA0422 \\
\hline NASA-4 & 45 & -36.84 & $<0.001$ & $<0.001$ & $<0.001$ & $<0.001$ & NASA0423 \\
\hline NASA-4 & 47 & -38.84 & $<0.001$ & $<0.001$ & $<0.001$ & $<0.001$ & NASA0424 \\
\hline NASA-4 & 49 & -40.84 & $<0.001$ & 0.023 & $<0.001$ & 0.002 & NASA0425 \\
\hline NASA-4 & 51.5 & -43.34 & 0.743 & 0.014 & $<0.001$ & $<0.001$ & NASA0426 \\
\hline
\end{tabular}




\begin{tabular}{|c|c|c|c|c|c|c|c|}
\hline \multirow[b]{2}{*}{ Loc. Id } & \multicolumn{2}{|c|}{ feet } & \multicolumn{4}{|c|}{ ug/kg } & \multirow[b]{2}{*}{ Samp. Id } \\
\hline & depth & elevation & $\mathrm{VCl}$ & $F-113$ & cis-DCE & TCE & \\
\hline NASA-5 & $\overline{1}$ & 7 & $<0.001$ & 0.191 & 0.004 & 0.002 & NASA0500 \\
\hline NASA-5 & 3 & 5 & 0.188 & 7.204 & 0.585 & 0.171 & NASA0501 \\
\hline NASA-5 & 5 & 3 & 0.463 & 15.767 & 2.711 & 0.481 & NASA0502 \\
\hline NASA-5 & $\overline{7}$ & 1 & 6.461 & 42.107 & 87.357 & 4.143 & NASA0503 \\
\hline NASA-5 & 9 & -1 & 4.251 & 21.816 & 45.315 & 2.108 & NASA0504 \\
\hline NASA-5 & 11 & -3 & 1.397 & 11.006 & 7.468 & 0.381 & NASA0505 \\
\hline NASA-5 & 13 & -5 & 2.774 & 0.541 & 1.463 & 0.021 & NASA0506 \\
\hline NASA-5 & 15 & -7 & 3.049 & 0.816 & 1.346 & 0.030 & NASA0507 \\
\hline NASA-5 & 17 & -9 & 3.493 & 0.099 & 0.936 & 0.005 & NASA0508 \\
\hline NASA-5 & 19 & -11 & 0.753 & 0.015 & 0.358 & $<0.001$ & NASA0509 \\
\hline NASA-5 & 21 & -13 & 1.522 & 1.267 & 1.081 & 0.054 & NASA0510 \\
\hline NASA-5 & 23 & -15 & 0.606 & 0.856 & 0.570 & 0.030 & NASA0511 \\
\hline NASA-5 & 25 & -17 & 0.285 & 1.309 & 0.750 & 0.161 & NASA0512 \\
\hline NASA-5 & 27 & -19 & 0.282 & 1.101 & 0.752 & 0.149 & NASA0513 \\
\hline NASA-5 & 29 & -21 & 0.473 & 1.314 & 0.857 & 0.792 & NASA0514 \\
\hline NASA-5 & 31 & -23 & 1.585 & 5.454 & 2.756 & 0.546 & NASA0515 \\
\hline NASA-5 & 34 & -26 & 0.222 & 1.069 & 0.721 & 0.310 & NASA0516 \\
\hline NASA-5 & 35 & -27 & $<0.001$ & 1.178 & 0.771 & 0.907 & NASA0517 \\
\hline NASA-5 & 37 & -29 & 0.076 & 1.270 & 0.998 & 1.698 & NASA0518 \\
\hline NASA-5 & 39 & -31 & 0.323 & 1.861 & 2.657 & 4.724 & NASA0519 \\
\hline NASA-5 & 41 & -33 & 0.191 & 0.787 & 0.711 & 0.574 & NASA0520 \\
\hline NASA-5 & 43 & -35 & 0.182 & $<0.001$ & $<0.001$ & $<0.001$ & NASA0521 \\
\hline NASA-5 & 45 & -37 & $<0.001$ & $<0.001$ & $<0.001$ & $<0.001$ & NASA0522 \\
\hline NASA-5 & 47 & -39 & $<0.001$ & $<0.001$ & $<0.001$ & $<0.001$ & NASA0523 \\
\hline NASA-5 & 49 & -41 & 0.586 & 1.572 & 1.584 & 0.908 & NASA0524 \\
\hline NASA-5 & 49.5 & -42 & $<0.001$ & $<0.001$ & $<0.001$ & $<0.001$ & NASA0525 \\
\hline NASA-6 & 1 & 5.32 & $<0.001$ & $<0.001$ & $<0.001$ & $<0.001$ & NASA0600 \\
\hline NASA-6 & 3 & 3.32 & $<0.001$ & $<0.001$ & $<0.001$ & $<0.001$ & NASA0601 \\
\hline NASA-6 & 5 & 1.32 & $<0.001$ & $<0.001$ & $<0.001$ & $<0.001$ & NASA0602 \\
\hline NASA-6 & 7 & -0.68 & $<0.001$ & $<0.001$ & $<0.001$ & $<0.001$ & NASA0603 \\
\hline NASA-6 & 9 & -2.68 & $<0.001$ & $<0.001$ & $<0.001$ & $<0.001$ & NASA0604 \\
\hline NASA-6 & 11 & -4.68 & $<0.001$ & $<0.001$ & $<0.001$ & $<0.001$ & NASA0605 \\
\hline NASA-6 & 13 & -6.68 & $<0.001$ & $<0.001$ & $<0.001$ & $<0.001$ & NASA0606 \\
\hline NASA-6 & 15 & -8.68 & $<0.001$ & $<0.001$ & $<0.001$ & $<0.001$ & NASA0607 \\
\hline NASA-6 & 17 & -10.68 & $<0.001$ & $<0.001$ & $<0.001$ & $<0.001$ & NASA0608 \\
\hline NASA-6 & 19 & -12.68 & $<0.001$ & $<0.001$ & $<0.001$ & $<0.001$ & NASA0609 \\
\hline NASA-6 & 21 & -14.68 & $<0.001$ & $<0.001$ & $<0.001$ & $<0.001$ & NASA0610 \\
\hline NASA-6 & 23 & -16.68 & $<0.001$ & $<0.001$ & $<0.001$ & $<0.001$ & NASA0611 \\
\hline NASA-6 & 25 & -18.68 & $<0.001$ & $<0.001$ & $<0.001$ & $<0.001$ & NASA0612 \\
\hline NASA-6 & 27 & -20.68 & $<0.001$ & $<0.001$ & 0.001 & $<0.001$ & NASA0613 \\
\hline NASA- 6 & 29 & -22.68 & $<0.001$ & $<0.001$ & $<0.001$ & $<0.001$ & NASA0614 \\
\hline NASA-6 & 31 & -24.68 & $<0.001$ & $<0.001$ & 0.003 & 0.002 & NASA0615 \\
\hline NASA-6 & 33 & -26.68 & $<0.001$ & $<0.001$ & 0.033 & $<0.001$ & NASA0616 \\
\hline NASA- 6 & 35 & -28.68 & $<0.001$ & $<0.001$ & $<0.001$ & $<0.001$ & NASA0617 \\
\hline NASA-6 & 37 & -30.68 & $<0.001$ & $<0.001$ & 0.065 & 0.002 & NASA0618 \\
\hline NASA-6 & 39 & -32.68 & 5.384 & $<0.001$ & 0.689 & $<0.001$ & NASA0619 \\
\hline NASA-6 & 41 & -34.68 & 5.832 & $<0.001$ & 1.101 & $<0.001$ & NASA0620 \\
\hline
\end{tabular}




\begin{tabular}{|c|c|c|c|c|c|c|c|}
\hline \multirow[b]{2}{*}{ Loc. Id } & \multicolumn{2}{|c|}{ feet } & \multicolumn{4}{|c|}{$\mathrm{ug} / \mathrm{kg}$} & \multirow[b]{2}{*}{ Samp. Id } \\
\hline & depth & elevation & $\mathrm{VCl}$ & $F-113$ & cis-DCE & TCE & \\
\hline NASA-6 & 43 & -36.68 & 1.982 & $<0.001$ & 0.444 & $<0.001$ & NASA0621 \\
\hline NASA- 6 & 45 & -38.68 & 2.015 & $<0.001$ & 0.412 & $<0.001$ & NASA0622 \\
\hline NASA-6 & 47 & -40.68 & $<0.001$ & $<0.001$ & $<0.001$ & $<0.001$ & NASA0623 \\
\hline NASA-6 & 48.5 & -42.18 & $<0.001$ & $<0.001$ & $<0.001$ & $<0.001$ & NASA0624 \\
\hline NASA-6 & 49.5 & -43.18 & 1.165 & $<0.001$ & $<0.001$ & $<0.001$ & NASA0625 \\
\hline NASA-7 & 1 & 6.24 & $<0.001$ & $<0.001$ & $<0.001$ & $<0.001$ & NASA0700 \\
\hline NASA-7 & 3 & 4.24 & $<0.001$ & $<0.001$ & $<0.001$ & $<0.001$ & NASA0701 \\
\hline NASA-7 & 5 & 2.24 & $<0.001$ & $<0.001$ & $<0.001$ & $<0.001$ & NASA0702 \\
\hline NASA-7 & 7 & 0.24 & $<0.001$ & $<0.001$ & 0.005 & $<0.001$ & NASA0703 \\
\hline NASA-7 & 9 & -1.76 & $<0.001$ & $<0.001$ & 0.014 & $<0.001$ & NASA0704 \\
\hline NASA-7 & 11 & -3.76 & $<0.001$ & $<0.001$ & 0.010 & $<0.001$ & NASA0705 \\
\hline NASA-7 & 13 & -5.76 & $<0.001$ & $<0.001$ & $<0.001$ & $<0.001$ & NASA0706 \\
\hline NASA-7 & 15 & -7.76 & $<0.001$ & $<0.001$ & 0.003 & $<0.001$ & NASA0707 \\
\hline NASA-7 & 17 & -9.76 & $<0.001$ & $<0.001$ & $<0.001$ & $<0.001$ & NASA0708 \\
\hline NASA-7 & 19 & -11.76 & $<0.001$ & $<0.001$ & $<0.001$ & $<0.001$ & NASA0709 \\
\hline NASA-7 & 21 & -13.76 & $<0.001$ & $<0.001$ & $<0.001$ & $<0.001$ & NASA0710 \\
\hline NASA-7 & 23 & -15.76 & $<0.001$ & $<0.001$ & $<0.001$ & $<0.001$ & NASA0711 \\
\hline NASA-7 & 25 & -17.76 & $<0.001$ & $<0.001$ & $<0.001$ & $<0.001$ & NASA0712 \\
\hline NASA-7 & 27 & -19.76 & $<0.001$ & $<0.001$ & $<0.001$ & $<0.001$ & NASA0713 \\
\hline NASA-7 & 29 & -21.76 & $<0.001$ & $<0.001$ & $<0.001$ & $<0.001$ & NASA0714 \\
\hline NASA-7 & 31 & -23.76 & $<0.001$ & $<0.001$ & $<0.001$ & $<0.001$ & NASA0715 \\
\hline NASA-7 & 33 & -25.76 & $<0.001$ & $<0.001$ & $<0.001$ & $<0.001$ & NASA0716 \\
\hline NASA-7 & 35 & -27.76 & $<0.001$ & $<0.001$ & 0.019 & 0.007 & NASA0717 \\
\hline NASA-7 & 37 & -29.76 & 2.894 & $<0.001$ & 0.143 & 0.003 & NASA0718 \\
\hline NASA-7 & 39 & -31.76 & $<0.001$ & $<0.001$ & $<0.001$ & $<0.001$ & NASA0719 \\
\hline NASA-7 & 41 & -33.76 & 2.296 & $<0.001$ & 0.173 & 0.001 & NASA0720 \\
\hline NASA-7 & 43 & -35.76 & 1.137 & $<0.001$ & 0.011 & $<0.001$ & NASA0721 \\
\hline NASA-7 & 45 & -37.76 & $<0.001$ & $<0.001$ & $<0.001$ & $<0.001$ & NASA0722 \\
\hline NASA-7 & 47 & -39.76 & $<0.001$ & $<0.001$ & $<0.001$ & $<0.001$ & NASA0723 \\
\hline NASA-7 & 48.5 & -41.26 & $<0.001$ & $<0.001$ & 0.030 & $<0.001$ & NASA0724 \\
\hline NASA-7 & 49.5 & -42.26 & $<0.001$ & $<0.001$ & $<0.001$ & $<0.001$ & NASA0725 \\
\hline NASA-9 & 15 & -8.03 & $<0.001$ & $<0.001$ & $<0.001$ & $<0.001$ & NASA0900 \\
\hline NASA-9 & 18 & -11.03 & $<0.001$ & $<0.001$ & $<0.001$ & $<0.001$ & NASA0901 \\
\hline NASA-9 & 20 & -13.03 & $<0.001$ & $<0.001$ & $<0.001$ & $<0.001$ & NASA0902 \\
\hline NASA-9 & 21 & -14.03 & $<0.001$ & $<0.001$ & $<0.001$ & $<0.001$ & NASA0903 \\
\hline NASA-9 & 23 & -16.03 & $<0.001$ & $<0.001$ & $<0.001$ & $<0.001$ & NASA0904 \\
\hline NASA-9 & 25 & -18.03 & $<0.001$ & $<0.001$ & $<0.001$ & $<0.001$ & NASA0905 \\
\hline NASA-9 & 27 & -20.03 & $<0.001$ & $<0.001$ & $<0.001$ & $<0.001$ & NASA0906 \\
\hline NASA-9 & 29 & -22.03 & $<0.001$ & $<0.001$ & $<0.001$ & $<0.001$ & NASA0907 \\
\hline NASA-9 & 31 & -24.03 & $<0.001$ & $<0.001$ & $<0.001$ & $<0.001$ & NASA0908 \\
\hline NASA-9 & 33 & -26.03 & $<0.001$ & $<0.001$ & $<0.001$ & $<0.001$ & NASA0909 \\
\hline NASA-9 & 35 & -28.03 & $<0.001$ & $<0.001$ & $<0.001$ & $<0.001$ & NASA0910 \\
\hline NASA-9 & 37.5 & -30.53 & $<0.001$ & $<0.001$ & $<0.001$ & $<0.001$ & NASA0911 \\
\hline NASA-9 & 40 & -33.03 & $<0.001$ & $<0.001$ & $<0.001$ & $<0.001$ & NASA0912 \\
\hline NASA-9 & 41 & -34.03 & $<0.001$ & $<0.001$ & $<0.001$ & $<0.001$ & NASA0913 \\
\hline NASA-9 & 43 & -36.03 & $<0.001$ & $<0.001$ & $<0.001$ & $<0.001$ & NASA0914 \\
\hline NASA-9 & 45 & -38.03 & $<0.001$ & $<0.001$ & $<0.001$ & $<0.001$ & NASA0915 \\
\hline
\end{tabular}




\begin{tabular}{|c|c|c|c|c|c|c|c|}
\hline \multirow[b]{2}{*}{ Loc. Id } & \multicolumn{2}{|c|}{ feet } & \multicolumn{4}{|c|}{ ug/kg } & \multirow[b]{2}{*}{ Samp. Id } \\
\hline & depth & elevation & $\mathrm{VCl}$ & $F-113$ & cis-DCE & TCE & \\
\hline NASA-9 & 47 & -40.03 & $<0.001$ & $<0.001$ & $<0.001$ & $<0.001$ & NASA0916 \\
\hline NASA-9 & 49 & -42.03 & $<0.001$ & $<0.001$ & $<0.001$ & $<0.001$ & NASA0917 \\
\hline NASA-9 & 49.5 & -42.53 & $<0.001$ & $<0.001$ & $<0.001$ & $<0.001$ & NASA0918 \\
\hline NASA-10 & 15 & -9.47 & $<0.001$ & $<0.001$ & $<0.001$ & $<0.001$ & NASA1000 \\
\hline NASA-10 & 17.5 & -11.97 & $<0.001$ & $<0.001$ & $<0.001$ & $<0.001$ & NASA1001 \\
\hline NASA-10 & 19.5 & -13.97 & $<0.001$ & $<0.001$ & $<0.001$ & $<0.001$ & NASA1002 \\
\hline NASA-10 & 21 & -15.47 & $<0.001$ & $<0.001$ & $<0.001$ & $<0.001$ & NASA1003 \\
\hline NASA-10 & 23 & -17.47 & $<0.001$ & $<0.001$ & $<0.001$ & $<0.001$ & NASA1004 \\
\hline NASA-10 & 25 & -19.47 & $<0.001$ & $<0.001$ & $<0.001$ & $<0.001$ & NASA1005 \\
\hline NASA-10 & 27 & -21.47 & $<0.001$ & $<0.001$ & $<0.001$ & $<0.001$ & NASA1006 \\
\hline NASA-10 & 29 & -23.47 & $<0.001$ & $<0.001$ & $<0.001$ & $<0.001$ & NASA 1007 \\
\hline NASA-10 & 31 & -25.47 & $<0.001$ & $<0.001$ & $<0.001$ & $<0.001$ & NASA1008 \\
\hline NASA-10 & 33 & -27.47 & $<0.001$ & $<0.001$ & $<0.001$ & $<0.001$ & NASA1009 \\
\hline NASA-10 & 35 & -29.47 & $<0.001$ & $<0.001$ & $<0.001$ & $<0.001$ & NASA1010 \\
\hline NASA-10 & 37 & -31.47 & $<0.001$ & $<0.001$ & $<0.001$ & $<0.001$ & NASA1011 \\
\hline NASA-10 & 39 & -33.47 & $<0.001$ & $<0.001$ & $<0.001$ & $<0.001$ & NASA1012 \\
\hline NASA-10 & 41 & -35.47 & $<0.001$ & $<0.001$ & $<0.001$ & $<0.001$ & NASA1013 \\
\hline NASA-10 & 43 & -37.47 & $<0.001$ & $<0.001$ & $<0.001$ & $<0.001$ & NASA1014 \\
\hline NASA-10 & 45 & -39.47 & $<0.001$ & $<0.001$ & $<0.001$ & $<0.001$ & NASA1015 \\
\hline NASA-10 & 47 & -41.47 & $<0.001$ & $<0.001$ & $<0.001$ & $<0.001$ & NASA1016 \\
\hline NASA-10 & 49.8 & -44.27 & $<0.001$ & $<0.001$ & $<0.001$ & $<0.001$ & NASA1017 \\
\hline NASA-10 & 50 & -44.47 & $<0.001$ & $<0.001$ & $<0.001$ & $<0.001$ & NASA1018 \\
\hline NASA-11 & 1 & 8.64 & $<0.001$ & $<0.001$ & $<0.001$ & $<0.001$ & NASA1100 \\
\hline NASA-11 & 3 & 6.64 & $<0.001$ & $<0.001$ & $<0.001$ & $<0.001$ & NASA1101 \\
\hline NASA-11 & 5 & 4.64 & $<0.001$ & $<0.001$ & $<0.001$ & $<0.001$ & NASA1102 \\
\hline NASA-11 & 7 & 2.64 & $<0.001$ & $<0.001$ & $<0.001$ & $<0.001$ & NASA1103 \\
\hline NASA-11 & 9 & 0.64 & $<0.001$ & $<0.001$ & $<0.001$ & $<0.001$ & NASA1104 \\
\hline NASA-11 & 11 & -1.36 & $<0.001$ & $<0.001$ & $<0.001$ & $<0.001$ & NASA1105 \\
\hline NASA-11 & 13 & -3.36 & $<0.001$ & $<0.001$ & $<0.001$ & $<0.001$ & NASA1106 \\
\hline NASA-11 & 15 & -5.36 & $<0.001$ & $<0.001$ & $<0.001$ & $<0.001$ & NASA1107 \\
\hline NASA-11 & 17 & -7.36 & $<0.001$ & $<0.001$ & $<0.001$ & $<0.001$ & NASA1108 \\
\hline NASA-11 & 19 & -9.36 & $<0.001$ & $<0.001$ & $<0.001$ & $<0.001$ & NASA1109 \\
\hline NASA-11 & 21 & -11.36 & $<0.001$ & $<0.001$ & $<0.001$ & $<0.001$ & NASA1110 \\
\hline NASA-11 & 23 & -13.36 & $<0.001$ & $<0.001$ & $<0.001$ & $<0.001$ & NASA1111 \\
\hline NASA-11 & 25 & -15.36 & $<0.001$ & $<0.001$ & $<0.001$ & $<0.001$ & NASA1112 \\
\hline NASA-11 & 27 & -17.36 & $<0.001$ & $<0.001$ & $<0.001$ & $<0.001$ & NASA 1113 \\
\hline NASA-11 & 29 & -19.36 & $<0.001$ & $<0.001$ & $<0.001$ & $<0.001$ & NASA1114 \\
\hline NASA-11 & 31 & -21.36 & $<0.001$ & $<0.001$ & $<0.001$ & $<0.001$ & NASA1115 \\
\hline NASA-11 & 33 & -23.36 & $<0.001$ & $<0.001$ & $<0.001$ & $<0.001$ & NASA1116 \\
\hline NASA-11 & 35 & -25.36 & $<0.001$ & $<0.001$ & $<0.001$ & $<0.001$ & NASA1117 \\
\hline NASA-11 & 37 & -27.36 & $<0.001$ & 0.002 & $<0.001$ & $<0.001$ & NASA1118 \\
\hline NASA-11 & 39 & -29.36 & $<0.001$ & $<0.001$ & $<0.001$ & $<0.001$ & NASA1119 \\
\hline NASA-11 & 41 & -31.36 & $<0.001$ & $<0.001$ & $<0.001$ & $<0.001$ & NASA1120 \\
\hline NASA-11 & 43 & -33.36 & $<0.001$ & $<0.001$ & $<0.001$ & $<0.001$ & NASA1121 \\
\hline NASA-11 & 45 & -35.36 & $<0.001$ & $<0.001$ & $<0.001$ & $<0.001$ & NASA1122 \\
\hline NASA-11 & 47 & -37.36 & $<0.001$ & $<0.001$ & $<0.001$ & $<0.001$ & NASA1123 \\
\hline NASA-11 & 49 & -39.36 & $<0.001$ & $<0.001$ & $<0.001$ & $<0.001$ & NASA1124 \\
\hline
\end{tabular}




\begin{tabular}{|c|c|c|c|c|c|c|c|}
\hline \multirow[b]{2}{*}{ Loc. Id } & \multicolumn{2}{|c|}{ feet } & \multicolumn{4}{|c|}{ ug/kg } & \multirow[b]{2}{*}{ Samp. Id } \\
\hline & depth & elevation & $\mathrm{VCl}$ & $\mathrm{F}-113$ & cis-DCE & TCE & \\
\hline NASA-11 & 50.5 & -40.86 & $<0.001$ & $<0.001$ & $<0.001$ & $<0.001$ & NASA1125 \\
\hline NASA-11 & 51.5 & -41.86 & $<0.001$ & 0.004 & $<0.001$ & 0.001 & NASA1126 \\
\hline NASA-14 & 1 & 7.52 & $<0.001$ & $<0.001$ & $<0.001$ & $<0.001$ & NASA1400 \\
\hline NASA-14 & 3 & 5.52 & $<0.001$ & $<0.001$ & $<0.001$ & $<0.001$ & NASA1401 \\
\hline NASA-14 & 5 & 3.52 & $<0.001$ & $<0.001$ & $<0.001$ & $<0.001$ & NASA1402 \\
\hline NASA-14 & 7 & 1.52 & $<0.001$ & $<0.001$ & $<0.001$ & $<0.001$ & NASA1403 \\
\hline NASA-14 & 9 & -0.48 & $<0.001$ & $<0.001$ & 0.046 & $<0.001$ & NASA1404 \\
\hline NASA-14 & 11 & -2.48 & $<0.001$ & $<0.001$ & $<0.001$ & $<0.001$ & NASA1405 \\
\hline NASA-14 & 13.5 & -4.98 & $<0.001$ & $<0.001$ & 0.023 & $<0.001$ & NASA1406 \\
\hline NASA-14 & 15 & -6.48 & $<0.001$ & $<0.001$ & $<0.001$ & $<0.001$ & NASA1407 \\
\hline NASA-14 & 17 & -8.48 & $<0.001$ & $<0.001$ & $<0.001$ & $<0.001$ & NASA1408 \\
\hline NASA-14 & 21 & -12.48 & 0.629 & $<0.001$ & 0.022 & 0.002 & NASA1409 \\
\hline NASA-14 & 23 & -14.48 & 0.925 & $<0.001$ & 0.223 & 0.003 & NASA1410 \\
\hline NASA-14 & 25 & -16.48 & 0.138 & $<0.001$ & 0.313 & 0.007 & NASA1411 \\
\hline NASA-14 & 27 & -18.48 & 0.019 & $<0.001$ & 0.510 & 0.016 & NASA1412 \\
\hline NASA-14 & 29 & -20.48 & $<0.001$ & $<0.001$ & 0.322 & 0.013 & NASA1413 \\
\hline NASA-14 & 31 & -22.48 & $<0.001$ & $<0.001$ & 0.057 & 0.002 & NASA1414 \\
\hline NASA-14 & 33 & -24.48 & $<0.001$ & $<0.001$ & 0.075 & 0.007 & NASA1415 \\
\hline NASA-14 & 35 & -26.48 & $<0.001$ & $<0.001$ & 0.043 & 0.002 & NASA1416 \\
\hline NASA-14 & 37 & -28.48 & $<0.001$ & $<0.001$ & 0.127 & 0.052 & NASA1417 \\
\hline NASA-14 & 39 & -30.48 & $<0.001$ & $<0.001$ & $<0.001$ & $<0.001$ & NASA1418 \\
\hline NASA-14 & 41 & -32.48 & 0.066 & $<0.001$ & 0.363 & 0.128 & NASA1419 \\
\hline NASA-14 & 43 & -34.48 & 1.442 & $<0.001$ & 0.443 & 0.164 & NASA1420 \\
\hline NASA-14 & 45 & -36.48 & 0.624 & $<0.001$ & 0.007 & 0.004 & NASA1421 \\
\hline NASA-14 & 47 & -38.48 & $<0.001$ & $<0.001$ & $<0.001$ & $<0.001$ & NASA1422 \\
\hline NASA-14 & 49 & -40.48 & $<0.001$ & $<0.001$ & $<0.001$ & $<0.001$ & NASA1423 \\
\hline NASA-14 & 51 & -42.48 & $<0.001$ & $<0.001$ & 0.046 & 0.021 & NASA1424 \\
\hline NASA-14 & 52.5 & -43.98 & $<0.001$ & $<0.001$ & $<0.001$ & $<0.001$ & NASA1425 \\
\hline NASA-14 & 53.5 & -44.98 & $<0.001$ & $<0.001$ & $<0.001$ & $<0.001$ & NASA1426 \\
\hline NASA-16 & 1 & 7.44 & $<0.001$ & $<0.001$ & $<0.001$ & $<0.001$ & NASA1600 \\
\hline NASA-16 & 3 & 5.44 & 0.088 & $<0.001$ & $<0.001$ & $<0.001$ & NASA1601 \\
\hline NASA-16 & 5 & 3.44 & $<0.001$ & $<0.001$ & $<0.001$ & $<0.001$ & NASA1602 \\
\hline NASA-16 & 7 & 1.44 & 1.071 & 1.421 & 0.277 & 0.093 & NASA1603 \\
\hline NASA-16 & 9 & -0.56 & 1.013 & $<0.001$ & 0.749 & $<0.001$ & NASA1604 \\
\hline NASA-16 & 11 & -2.56 & $<0.001$ & 0.030 & 0.063 & 0.017 & NASA1605 \\
\hline NASA-16 & 13 & -4.56 & $<0.001$ & 0.009 & 0.018 & 0.006 & NASA1606 \\
\hline NASA-16 & 15 & -6.56 & 0.765 & 0.006 & 0.595 & 0.009 & NASA1607 \\
\hline NASA-16 & 17 & -8.56 & 0.386 & 0.029 & 0.387 & 0.021 & NASA1608 \\
\hline NASA-16 & 19.75 & -11.31 & $<0.001$ & $<0.001$ & $<0.001$ & $<0.001$ & NASA1609 \\
\hline NASA-16 & 21 & -12.56 & $<0.001$ & $<0.001$ & $<0.001$ & $<0.001$ & NASA1610 \\
\hline NASA-16 & 23 & -14.56 & $<0.001$ & $<0.001$ & 0.007 & $<0.001$ & NASA1611 \\
\hline NASA-16 & 25 & -16.56 & $<0.001$ & $<0.001$ & $<0.001$ & $<0.001$ & NASA1612 \\
\hline NASA-16 & 27.5 & -19.06 & 0.002 & $<0.001$ & $<0.001$ & $<0.001$ & NASA1613 \\
\hline NASA-16 & 29 & -20.56 & $<0.001$ & $<0.001$ & $<0.001$ & $<0.001$ & NASA1614 \\
\hline NASA-16 & 31 & -22.56 & 0.197 & $<0.001$ & $<0.001$ & $<0.001$ & NASA1615 \\
\hline NASA-16 & 33 & -24.56 & $<0.001$ & $<0.001$ & $<0.001$ & $<0.001$ & NASA1616 \\
\hline NASA-16 & 35 & -26.56 & $<0.001$ & $<0.001$ & $<0.001$ & $<0.001$ & NASA1617 \\
\hline
\end{tabular}




\begin{tabular}{|c|c|c|c|c|c|c|c|}
\hline \multirow[b]{2}{*}{ Loc. Id } & \multicolumn{2}{|c|}{ feet } & \multicolumn{4}{|c|}{ ug/kg } & \multirow[b]{2}{*}{ Samp. Id } \\
\hline & depth & elevation & $\mathrm{VCl}$ & $\mathrm{F}-113$ & cis-DCE & TCE & \\
\hline NASA-16 & 37 & -28.56 & $<0.001$ & $<0.001$ & $<0.001$ & $<0.001$ & NASA1618 \\
\hline NASA-16 & 39 & -30.56 & 0.148 & $<0.001$ & $<0.001$ & $<0.001$ & NASA1619 \\
\hline NASA-16 & 41 & -32.56 & $<0.001$ & $<0.001$ & $<0.001$ & $<0.001$ & NASA1620 \\
\hline NASA-16 & 43 & -34.56 & 0.188 & $<0.001$ & $<0.001$ & $<0.001$ & NASA1621 \\
\hline NASA-16 & 45 & -36.56 & $<0.001$ & $<0.001$ & $<0.001$ & $<0.001$ & NASA1622 \\
\hline NASA-16 & 47 & -38.56 & $<0.001$ & $<0.001$ & $<0.001$ & $<0.001$ & NASA1623 \\
\hline NASA-16 & 48.5 & -40.06 & $<0.001$ & $<0.001$ & 0.007 & $<0.001$ & NASA1624 \\
\hline NASA-16 & 49.5 & -41.06 & 0.129 & $<0.001$ & $<0.001$ & $<0.001$ & NASA1625 \\
\hline NASA-17 & 15 & -10.01 & $<0.001$ & $<0.001$ & $<0.001$ & $<0.001$ & NASA1700 \\
\hline NASA-17 & 17 & -12.01 & $<0.001$ & $<0.001$ & $<0.001$ & $<0.001$ & NASA1701 \\
\hline NASA-17 & 19 & -14.01 & $<0.001$ & $<0.001$ & $<0.001$ & $<0.001$ & NASA1702 \\
\hline NASA-17 & 21 & -16.01 & $<0.001$ & $<0.001$ & $<0.001$ & $<0.001$ & NASA1703 \\
\hline NASA-17 & 23 & -18.01 & $<0.001$ & $<0.001$ & $<0.001$ & $<0.001$ & NASA1704 \\
\hline NASA-17 & 25 & -20.01 & $<0.001$ & $<0.001$ & $<0.001$ & $<0.001$ & NASA1705 \\
\hline NASA-17 & 27 & -22.01 & $<0.001$ & $<0.001$ & $<0.001$ & $<0.001$ & NASA1706 \\
\hline NASA-17 & 29 & -24.01 & $<0.001$ & $<0.001$ & $<0.001$ & $<0.001$ & NASA1707 \\
\hline NASA-17 & 31 & -26.01 & $<0.001$ & $<0.001$ & $<0.001$ & $<0.001$ & NASA1708 \\
\hline NASA-17 & 33 & -28.01 & $<0.001$ & $<0.001$ & $<0.001$ & $<0.001$ & NASA1709 \\
\hline NASA-17 & 35 & -30.01 & $<0.001$ & $<0.001$ & $<0.001$ & $<0.001$ & NASA1710 \\
\hline NASA-17 & 37 & -32.01 & $<0.001$ & $<0.001$ & $<0.001$ & $<0.001$ & NASA1711 \\
\hline NASA-17 & 39 & -34.01 & $<0.001$ & $<0.001$ & $<0.001$ & $<0.001$ & NASA1712 \\
\hline NASA-17 & 41 & -36.01 & $<0.001$ & 0.003 & $<0.001$ & $<0.001$ & NASA1713 \\
\hline NASA-17 & 43 & -38.01 & $<0.001$ & $<0.001$ & $<0.001$ & $<0.001$ & NASA1714 \\
\hline NASA-17 & 45 & -40.01 & $<0.001$ & $<0.001$ & $<0.001$ & $<0.001$ & NASA1715 \\
\hline NASA-17 & 47 & -42.01 & $<0.001$ & $<0.001$ & $<0.001$ & $<0.001$ & NASA1716 \\
\hline NASA-17 & 49.3 & -44.31 & $<0.001$ & $<0.001$ & $<0.001$ & $<0.001$ & NASA1717 \\
\hline NASA-17 & 49.8 & -44.81 & $<0.001$ & $<0.001$ & $<0.001$ & $<0.001$ & NASA1718 \\
\hline NASA-18 & 1 & 7.46 & $<0.001$ & 0.001 & $<0.001$ & $<0.001$ & NASA1800 \\
\hline NASA-18 & 3 & 5.46 & $<0.001$ & $<0.001$ & $<0.001$ & $<0.001$ & NASA1801 \\
\hline NASA-18 & 5 & 3.46 & $<0.001$ & $<0.001$ & $<0.001$ & $<0.001$ & NASA1802 \\
\hline NASA-18 & 7 & 1.46 & $<0.001$ & 1.297 & 0.009 & $<0.001$ & NASA1803 \\
\hline NASA-18 & 9 & -0.54 & $<0.001$ & 0.083 & 0.030 & $<0.001$ & NASA1804 \\
\hline NASA-18 & 11 & -2.54 & $<0.001$ & 0.007 & 0.023 & $<0.001$ & NASA1805 \\
\hline NASA-18 & 13 & -4.54 & $<0.001$ & 0.122 & 0.012 & $<0.001$ & NASA1806 \\
\hline NASA-18 & 15 & -6.54 & $<0.001$ & $<0.001$ & $<0.001$ & $<0.001$ & NASA1807 \\
\hline NASA-18 & 17 & -8.54 & $<0.001$ & $<0.001$ & $<0.001$ & $<0.001$ & NASA1808 \\
\hline NASA-18 & 19 & -10.54 & $<0.001$ & $<0.001$ & $<0.001$ & $<0.001$ & NASA1809 \\
\hline NASA-18 & 21 & -12.54 & $<0.001$ & $<0.001$ & $<0.001$ & $<0.001$ & NASA1810 \\
\hline NASA-18 & 23 & -14.54 & $<0.001$ & $<0.001$ & $<0.001$ & $<0.001$ & NASA1811 \\
\hline NASA-18 & 25 & -16.54 & $<0.001$ & $<0.001$ & $<0.001$ & $<0.001$ & NASA1812 \\
\hline NASA-18 & 27 & -18.54 & $<0.001$ & $<0.001$ & $<0.001$ & $<0.001$ & NASA1813 \\
\hline NASA-18 & 29 & -20.54 & $<0.001$ & 0.005 & $<0.001$ & $<0.001$ & NASA1814 \\
\hline NASA-18 & 31 & -22.54 & $<0.001$ & 0.002 & $<0.001$ & $<0.001$ & NASA1815 \\
\hline NASA-18 & 33 & -24.54 & $<0.001$ & $<0.001$ & $<0.001$ & $<0.001$ & NASA1816 \\
\hline NASA-18 & 35 & -26.54 & $<0.001$ & $<0.001$ & $<0.001$ & $<0.001$ & NASA1817 \\
\hline NASA-18 & 37 & -28.54 & $<0.001$ & 0.002 & $<0.001$ & $<0.001$ & NASA1818 \\
\hline NASA-18 & 39 & -30.54 & $<0.001$ & 0.008 & $<0.001$ & $<0.001$ & NASA1819 \\
\hline
\end{tabular}




\begin{tabular}{|c|c|c|c|c|c|c|c|}
\hline \multirow[b]{2}{*}{ Loc. Id } & \multicolumn{2}{|c|}{ feet } & \multicolumn{4}{|c|}{$\mathrm{ug} / \mathrm{kg}$} & \multirow[b]{2}{*}{ Samp. Id } \\
\hline & depth & elevation & $\mathrm{VCl}$ & $\mathrm{F}-113$ & cis-DCE & TCE & \\
\hline NASA-18 & 41 & -32.54 & $<0.001$ & $<0.001$ & $<0.001$ & $<0.001$ & NASA1820 \\
\hline NASA-18 & 43 & -34.54 & $<0.001$ & $<0.001$ & $<0.001$ & $<0.001$ & NASA1821 \\
\hline NASA-18 & 45 & -36.54 & $<0.001$ & $<0.001$ & $<0.001$ & $<0.001$ & NASA1822 \\
\hline NASA-18 & 47 & -38.54 & $<0.001$ & $<0.001$ & $<0.001$ & $<0.001$ & NASA1823 \\
\hline NASA-18 & 49 & -40.54 & $<0.001$ & $<0.001$ & $<0.001$ & $<0.001$ & NASA1824 \\
\hline NASA-18 & 51.5 & -43.04 & $<0.001$ & $<0.001$ & $<0.001$ & $<0.001$ & NASA1825 \\
\hline NASA-18 & 50.5 & -42.04 & $<0.001$ & $<0.001$ & $<0.001$ & $<0.001$ & NASA1826 \\
\hline NASA-19 & 1 & 6.15 & $<0.001$ & $<0.001$ & $<0.001$ & $<0.001$ & NASA1900 \\
\hline NASA-19 & 3 & 4.15 & $<0.001$ & $<0.001$ & $<0.001$ & $<0.001$ & NASA1901 \\
\hline NASA-19 & 5 & 2.15 & $<0.001$ & $<0.001$ & $<0.001$ & $<0.001$ & NASA1902 \\
\hline NASA-19 & 7 & 0.15 & $<0.001$ & $<0.001$ & $<0.001$ & $<0.001$ & NASA1903 \\
\hline NASA-19 & 9 & -1.85 & $<0.001$ & $<0.001$ & $<0.001$ & $<0.001$ & NASA1904 \\
\hline NASA-19 & 11 & -3.85 & $<0.001$ & $<0.001$ & $<0.001$ & $<0.001$ & NASA1905 \\
\hline NASA-19 & 15 & -7.85 & $<0.001$ & $<0.001$ & $<0.001$ & $<0.001$ & NASA1906 \\
\hline NASA-19 & 17 & -9.85 & $<0.001$ & $<0.001$ & $<0.001$ & $<0.001$ & NASA1907 \\
\hline NASA-19 & 19 & -11.85 & $<0.001$ & $<0.001$ & $<0.001$ & $<0.001$ & NASA1908 \\
\hline NASA-19 & 21 & -13.85 & $<0.001$ & $<0.001$ & $<0.001$ & $<0.001$ & NASA1909 \\
\hline NASA-19 & 23 & -15.85 & $<0.001$ & $<0.001$ & $<0.001$ & $<0.001$ & NASA1910 \\
\hline NASA-19 & 25 & -17.85 & $<0.001$ & $<0.001$ & $<0.001$ & $<0.001$ & NASA1911 \\
\hline NASA-19 & 27 & -19.85 & $<0.001$ & $<0.001$ & $<0.001$ & $<0.001$ & NASA1912 \\
\hline NASA-19 & 29 & -21.85 & $<0.001$ & $<0.001$ & $<0.001$ & $<0.001$ & NASA1913 \\
\hline NASA-19 & 31 & -23.85 & $<0.001$ & $<0.001$ & $<0.001$ & $<0.001$ & NASA1914 \\
\hline NASA-19 & 33 & -25.85 & $<0.001$ & $<0.001$ & $<0.001$ & $<0.001$ & NASA1915 \\
\hline NASA-19 & 35 & -27.85 & $<0.001$ & $<0.001$ & $<0.001$ & $<0.001$ & NASA1916 \\
\hline NASA-19 & 37 & -29.85 & $<0.001$ & $<0.001$ & $<0.001$ & $<0.001$ & NASA1917 \\
\hline NASA-19 & 39 & -31.85 & $<0.001$ & $<0.001$ & $<0.001$ & $<0.001$ & NASA1918 \\
\hline NASA-19 & 41 & -33.85 & $<0.001$ & $<0.001$ & $<0.001$ & $<0.001$ & NASA1919 \\
\hline NASA-19 & 43 & -35.85 & $<0.001$ & $<0.001$ & $<0.001$ & $<0.001$ & NASA1920 \\
\hline NASA-19 & 45 & -37.85 & $<0.001$ & $<0.001$ & $<0.001$ & $<0.001$ & NASA1921 \\
\hline NASA-19 & 47 & -39.85 & $<0.001$ & $<0.001$ & $<0.001$ & $<0.001$ & NASA1922 \\
\hline NASA-19 & 49 & -41.85 & $<0.001$ & $<0.001$ & $<0.001$ & $<0.001$ & NASA1923 \\
\hline
\end{tabular}

